\title{
Advances in Gaussian random field generation: A review
}

\author{
Yang Liu · Jingfa Li · Shuyu Sun* • Bo Yu
}

Received: date / Accepted: date

\begin{abstract}
Gaussian (normal) distribution is a basic continuous probability distribution in statistics, it plays a substantial role in scientific and engineering problems that related to stochastic phenomena. This paper aims to review state of the art of Gaussian random field generation methods, their applications in scientific and engineering issues of interest, and open-source software/packages for Gaussian random field generation. To this end, first we briefly introduce basic mathematical concepts and theories in Gaussian random field, then seven commonly-used Gaussian random field generation methods are systematically presented. The basic idea, mathematical framework of each generation method are introduced in detail and comparisons of these methods are summarized. Then representative applications of Gaussian random field in various areas, especially of engineering interest in recent two decades, are reviewed. For readers' convenience, four representative example codes are provided, and several relevant up-to-date open-source software and packages that freely available from the Internet are introduced.
\end{abstract}

Keywords Random field generation · Gaussian distribution · Numerical methods · Applications of Gaussian random field · Software and packages

Yang Liu, Jingfa Li, Shuyu Sun*

Computational Transport Phenomena Laboratory, Division of Physical Science and Engineering, King Abdullah University of Science and Technology, Thuwal 23955-6900, Saudi Arabia E-mail: yang.liu.3@kaust.edu.sa, jingfa.li@kaust.edu.sa, shuyu.sun@kaust.edu.sa

Jingfa Li, Bo Yu

School of Mechanical Engineering, Beijing Key Laboratory of Pipeline Critical Technology and Equipment for Deepwater Oil \& Gas Development, Beijing Institute of Petrochemical Technology, Beijing 102617, China E-mail: jingfa.li@kaust.edu.sa, yubobox@vip.163.com

* Corresponding author : Shuyu Sun 


\section{Introduction}

In nature and engineering, uncertainties are ubiquitous at various spatial and temporal scales, e.g., material properties, concrete structures, aquifers. These uncertainties may exert crucial impacts on the overall performance of physical processes. For example, in geotechnical design and analysis, the uncertainties of geometric shape, material properties, and load can cause unfavorable and unexpected responses. Fortunately, the presence of these uncertainties now has been widely acknowledged by the research community. How to describe and account for uncertainties in scientific and engineering issues of interest has been a research hot spot in recent 30 years.

Random fields are widely used to model the uncertainties, such as measurement errors, random fluctuations in geometric or material properties, human height distributions, etc. By treating these uncertainties as random variables or random fields, statistics-based analyses can be found in a consistent manner $[123,138,124]$. Previous studies indicate that one of the most significant concerns of stochastic simulations is efficiently and accurately generating suitable random fields to represent the uncertainties. These fields can be one-dimensional or multi-dimensional, homogeneous or non-homogeneous, stationary or non-stationary, one-variate or multi-variate, and Gaussian or nonGaussian, etc. Generally, statistical measures, e.g., mean, variance, and probability distribution function, are necessary to describe the random field. Especially, the selection of probability distribution function has significant influence on resulted random fields. Among commonly-used probability functions, the Gaussian distribution is the most fundamental distribution in statistics. The central limit theorem states that the average of independent and identically distributed random variables converges in distribution to the Gaussian distribution. Compared with other commonly-used distributions, the Gaussian distribution offers the advantages of flexible shape in data fitting by controlling the mean and variance. In addition, the Gaussian distribution has wellestablished properties for computer-aided simulations, for instance, R packages, Matlab and other developed softwares that can easily generate Gaussian random variables and fields. Furthermore, existing approaches for generating the non-Gaussian random field typically utilize the translation process theory to an underlying Gaussian random field. These features make the Gaussian distribution (and its log form) to be a better alternative to other distributions in many physical and social attributes.

Due to the importance of Gaussian random field, its generation methods have attracted a large number of researchers to devote to this topic, and it has been widely applied to engineering of interest [37]. For example, petroleum engineering experts apply the random field to describe the permeability and porosity of the reservoir porous media in subsurface flow and transport; ecologists use random landscapes as a basis for comparison against observations; earth scientists generate random features to simulate partially observable entities such as clouds or simulate subgrid land surface characteristics based on physical attributes, etc. In Fig. 1, we present the statistical data of the litera- 
ture containing keywords 'Random field generation' from 1970 to 2018 that obtained from Google scholar on October 1, 2018. It can be clearly seen that the random field generation has received increasing attentions from 1990s, which validates that studies on the random field generation or related topics have been the research hot spot. In addition, some representative books and reports on the random field generation were published and have been the important references in this field, such as the works of Vanmarcke (1983)[128], Gutjahr (1989)[47], Christakos (1992)[16], Zhang (2001)[135], Müller (2007)[88], Adler (2007)[2], Fenton (2008)[33]. As shown in Fig. 1, there are a large body of

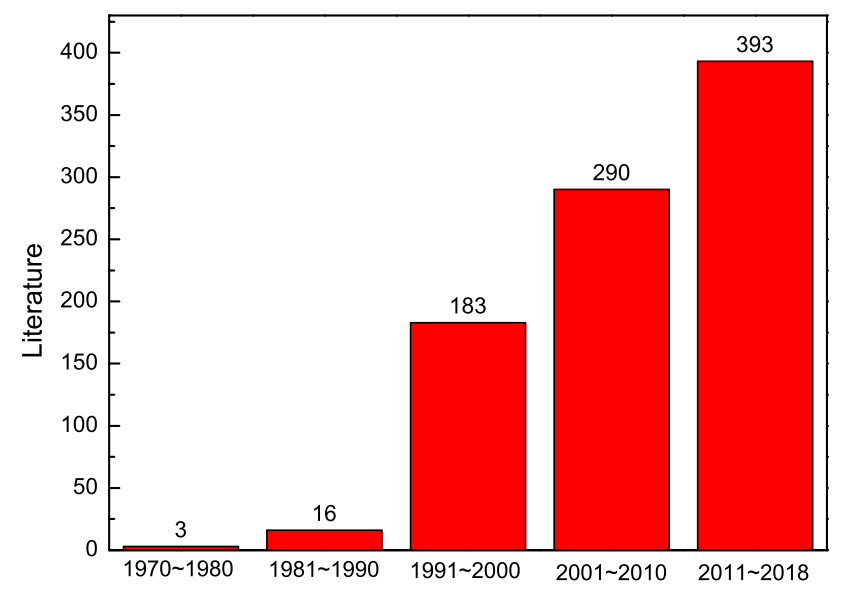

Fig. 1 Statistical data of the literature containing keywords "Random field generation" from 1970 to 2018 (data from Google scholar on October 1, 2018)

studies on random field generation techniques, to the best of the authors' knowledge, however, in these previous studies there are not much work introducing popular Gaussian random field generation methods comprehensively, or the review work only gave attentions to the random field generation methods proposed two decades ago [1]. Based on the background, the aim of this paper is to provide a comprehensive review on popular random field generation methods following Gaussian (and lognormal) distribution. Among the random field generation methods, we roughly checked the literature counted in Fig. 1 and found most of them are covered by seven methods (see percentage in Table 1 ), namely turning bands method, spectral method, matrix decomposition method, Karhunen-Loève expansion, moving average method, sequential simulation, and local average subdivision. Thus in this review we only focus on these seven representative Gaussian random field generation methods. This review also presents state-of-the-art representative applications of Gaussian 
random field in recent two decades and open-source software/packages for Gaussian random field generation available from the Internet, which may benefit scholars and engineers in various disciplines who are conducting research on stochastic phenomena.

Table 1 Percentage of papers that are covered by seven representative random field generation methods

\begin{tabular}{llllll}
\hline Years & $1970-1980$ & $1981-1990$ & $1991-2000$ & $2001-2010$ & $2011-2018$ \\
\hline Percentage & $67 \%$ & $75 \%$ & $70 \%$ & $66 \%$ & $60 \%$ \\
\hline
\end{tabular}

The remaining parts of this paper are organized as follows. In Section 2, the basic mathematical concepts in random field theory are first briefly introduced, then seven widely-used Gaussian random field generation methods are described in detail regarding the basic idea and mathematical foundation; comparisons of these methods are summarized from different aspects as well. In Section 3, representative applications of Gaussian random field in various scientific and engineering issues of interest in recent two decades are briefly reviewed, and several interesting random field results are reprinted and illustrated. In Section 4, an attempt is made to introduce the up-to-date opensource software and packages for Gaussian random field generation that are freely available from the Internet. The summary of this work is presented in Section 5.

\section{Methods in Gaussian Random Field Generation}

In this Section, we will first formulate the mathematics model, namely Gaussian random field. Then we will systematically review seven popular simulation methods for Gaussian random field generation in terms of the basic idea, mathematical derivation, implementation, and comparison of the advantages and limitations of each method.

\subsection{Mathematical Essential}

A random variable $X$ defined on a probability triple $(\Theta, \Sigma, P)$ maps a random event $\theta \in \Theta$ to real value in $\mathbb{R}^{n}$. A stochastic process is a collection of random variables $\left\{K: K(\theta, t)=X_{t}(\theta),\left(t \in \mathbb{R}^{n}\right)\right\}$, where $t$ is the index, typically it can indicate time or space. In this review, for brevity but without loss of generality, we focus on the latter case, where the stochastic process (random field) is a collection of random variables defined over a spatial domain.

Consider a stochastic process $K$ on a physical domain $\Omega$, for simplicity it is assumed to be square integrable,

$$
(K: \Omega \times \Theta \rightarrow \mathbb{R}) \in L_{2}(\Omega) \times L_{2}(\Theta, P) .
$$


Statistical properties are used to depict the random field. In many cases we are interested in stationary (or even isotropic) Gaussian stochastic processes, which is fully characterised by its mean and covariance function,

$$
\begin{aligned}
\mathbb{E}[K(x, \cdot)] & =\int_{\Theta} K(x, \theta) d P(\theta)=\mu, \quad \forall x \in \Omega, \\
C(x, y) & =\mathbb{E}[K(x, \cdot) K(y, \cdot)]=f(x-y) .
\end{aligned}
$$

The mean value $\mu$ is a constant over the field, the covariance function only depends on the difference of two indices (in isotropic case, only depends on the distance of two spatial locations). Without loss of generality, in this Section we will talk about the random process with mean value zero $(\mu=0)$.

There exist many choices for the covariance function, one general choice is called Matérn covariance,

$$
C_{\nu}(d)=\sigma^{2} \frac{2^{1-\nu}}{\Gamma(\nu)}\left(\sqrt{2 \nu} \frac{d}{\rho}\right)^{\nu} K_{\nu}\left(\sqrt{2 \nu} \frac{d}{\rho}\right),
$$

where $\Gamma$ is gamma function, $K_{\nu}$ is the modified Bessel function of the second kind, $\rho$ and $\nu$ are non-negative parameters. A variety of them correspond a set of random fields with various smoothness. Thus, this type of covariance function is able to model a large number of situations.

It should be noted that $\nu=\frac{1}{2}$ yields exponential covariance,

$$
C_{1 / 2}(d)=\sigma^{2} \exp \left(-\frac{d}{\rho}\right),
$$

while $\nu=+\infty$ yields Gaussian covariance,

$$
C_{+\infty}(d)=\sigma^{2} \exp \left(-\frac{d^{2}}{2 \rho^{2}}\right) .
$$

There also exist other choices of covariance kernel, such as linear covariance,

$$
C(d)=\left\{\begin{array}{cc}
\sigma^{2}\left(1-\frac{d}{\rho}\right), & d \leq \rho, \\
0, & \text { otherwise }
\end{array}\right.
$$

circular covariance,

$$
C(d)=\left\{\begin{array}{cc}
\sigma^{2}\left(1-\frac{2 d}{\rho \pi} \sqrt{1-\frac{d^{2}}{\rho^{2}}}-\frac{2}{\pi} \arcsin \left(\frac{d}{a}\right)\right), & d \leq \rho, \\
0, & \text { otherwise }
\end{array}\right.
$$

spherical covariance,

$$
C(d)=\left\{\begin{array}{cc}
\sigma^{2}\left(1-\frac{3 d}{2 \rho}-\frac{d^{3}}{2 \rho^{3}}\right), & d \leq \rho, \\
0, & \text { otherwise }
\end{array}\right.
$$

Whittle covariance,

$$
C(d)=\sigma^{2} \frac{d}{\rho} K_{1}\left(\frac{d}{\rho}\right)
$$


and many other ad hoc choices.

Now that we have formulate the basis of a stationary (isotropic) Gaussian random field, in the following we plan to introduce seven popular methods to generalize realizations of the random field $K$. Owing to the limitation of our knowledge, we here only provide a biased collection of random field generation methods.

\subsection{Turning Bands Method}

Turning bands method (TBM) was first proposed by Matheron in 1973 [85], where the method was applied to simulate a random field. The method was then developed and improved by other researchers $[83,81,82,127,53,54$, 27]. For example, Mantoglou[83,81,82] studied both 2-D and 3-D in isotropic and anisotropic cases, Tompson[127] suggested the use of more random lines in 3 -D and also revealed the improved performance by employing a fast Fourier transform in the generation of line process. The idea of TBM is to represent the random process in $\mathbb{R}^{2}$ or $\mathbb{R}^{3}$ by a number of independent 1 -D processes anchored at a same point in space, while the function value at a arbitrary point in space can be found by projecting the point onto each line process, see Fig. 2. The line process must preserve the covariance structure, in a way that the covariance function $C_{1}(\xi)$ of the 1-D line process corresponds the original covariance function $C(d)$. Mantoglou [83] gave a detailed illustration in both 2 -D and 3-D cases.

Let $K$ denote the isotropic Gaussian random field in $\mathbb{R}^{n}(n=2,3)$ with mean zero and covariance function $C(d)$, where $d$ is the spatial distance of two points. TBM first selects an origin in $\mathbb{R}^{n}$, from which the $L$ independent line processes $K_{i}$ are generated, $i=1,2, \cdots, L$. The orientation of the lines are uniformly distributed in $n$-D unit ball centered at the origin. Take the point of interest $x_{n}$ as an example, the projection of the point onto each of the line is $x_{n} \cdot u_{i}$, where $u_{i}$ is the unit vector on line $i$. Then the value $K\left(x_{n}\right)$ is given by,

$$
K\left(x_{n}\right)=\frac{1}{\sqrt{L}} \sum_{i=1}^{L} K_{i}\left(x_{n} \cdot u_{i}\right) .
$$

It should be noted that on each line, the discrete random process is generated. In a small "bandwidth", the random process assumes the same value. The lines are generated around the origin, which seems to turn around. Thus the method was named as "Turning Bands Method" [85].

As mentioned earlier, TBM must preserve the original covariance structure. This means that the covariance of two points $x_{1}, x_{2}$ in the turning bands simulated field must be the same as in the original field, i.e.,

$$
\begin{aligned}
C\left(x_{1}, x_{2}\right) & =\mathbb{E}\left[K\left(x_{1}\right) K\left(x_{2}\right)\right] \\
& =\frac{1}{L} \sum_{i=1}^{L} \sum_{j=1}^{L} \mathbb{E}\left[K_{i}\left(x_{1} \cdot u_{i}\right) K_{j}\left(x_{2} \cdot u_{j}\right)\right] .
\end{aligned}
$$




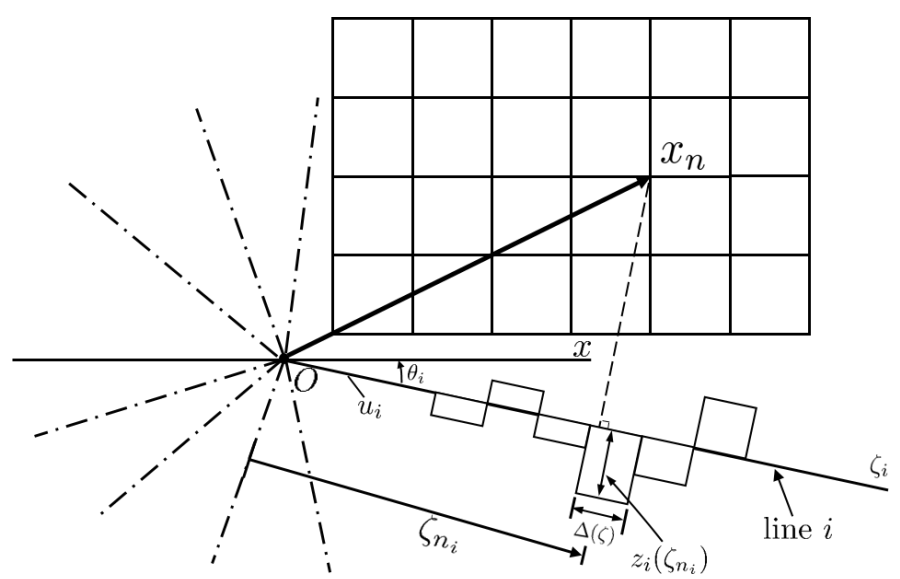

Fig. 2 An illustration of turning bands method, Mantoglou and Wilson (1982) [83]

Notice that the independence of each line, the above equation can be written as,

$$
\begin{aligned}
C\left(x_{1}, x_{2}\right) & =\frac{1}{L} \sum_{i=1}^{L} \mathbb{E}\left[K_{i}\left(x_{1} \cdot u_{i}\right) K_{i}\left(x_{2} \cdot u_{i}\right)\right] \\
& =\frac{1}{L} \sum_{i=1}^{L} C_{1}\left(h \cdot u_{i}\right)
\end{aligned}
$$

where $h=x_{2}-x_{1}$. Now let $L \rightarrow \infty$,

$$
\begin{aligned}
C\left(x_{1}, x_{2}\right) & =\lim _{L \rightarrow \infty} \frac{1}{L} \sum_{i=1}^{L} C_{1}\left(h \cdot u_{i}\right) \\
& =\mathbb{E}\left[C_{1}\left(h \cdot u_{i}\right)\right] \\
& =\int_{\partial B} C_{1}(h \cdot u) f(u) d u
\end{aligned}
$$

where $\partial B$ is the boundary of unit hypersphere, $f(u)$ is the uniform probability density function on the unit-ball surface of the unit vector $u$. In $2-\mathrm{D}$ and 3-D cases, $f(u)=1 / 2 \pi$ and $1 / 4 \pi$ respectively.

\subsubsection{Corresponding Line Process}

In the 3 -D case, set polar coordinate $(r, \theta, \varphi)$ and let the zenith-axis be aligned with $h=x_{2}-x_{1}$ direction, see Fig. 3 . It is obvious that $h \cdot u=r \cos \varphi$, 
$d u=\sin \varphi d \varphi d \theta$ and $f(u)=1 / 4 \pi$, then,

$$
\begin{aligned}
C(r) & =\frac{1}{4 \pi} \int_{0}^{2 \pi} \int_{0}^{\pi} C_{1}(r \cos \varphi) \sin \varphi d \varphi d \theta \\
& =\frac{1}{2} \int_{0}^{\pi} C_{1}(r \cos \varphi) \sin \varphi d \varphi \\
& =\int_{0}^{\pi / 2} C_{1}(r \cos \varphi) \sin \varphi d \varphi .
\end{aligned}
$$

The last step comes from the fact that $C_{1}$ is an even function. Now let

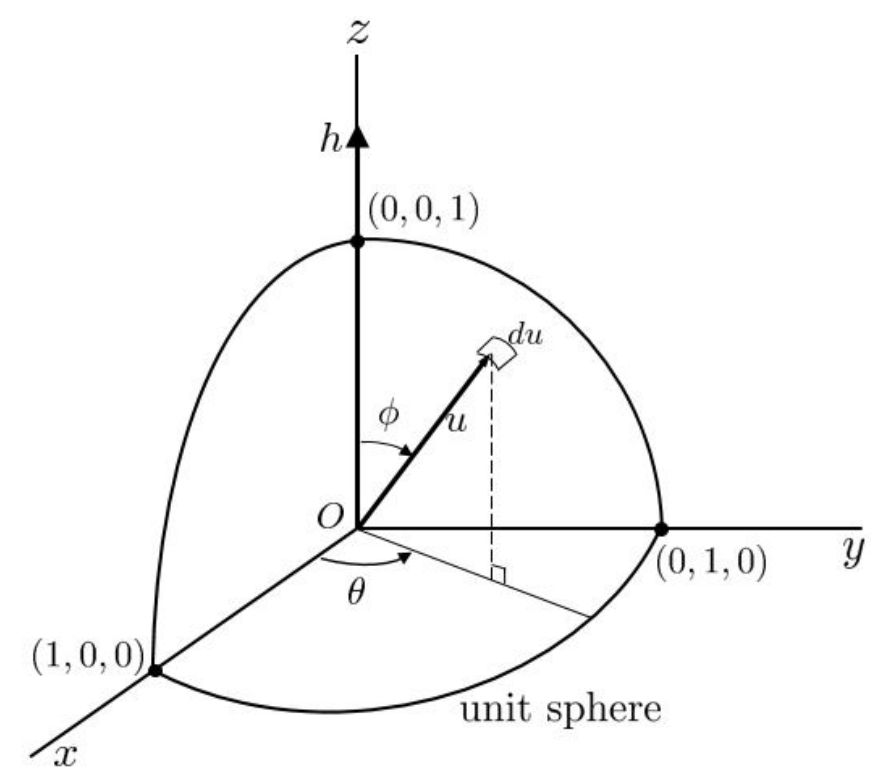

Fig. 3 Integration in unit sphere, Mantoglou and Wilson (1982) [83]

$\zeta=r \cos \varphi$, which is the coordinate on line $i$. Then,

$$
\begin{aligned}
C(r) & =\int_{0}^{1} C_{1}(\zeta) d(\zeta / r) \\
& =\frac{1}{r} \int_{0}^{r} C_{1}(\zeta) d \zeta .
\end{aligned}
$$

The above equation can be expressed in a more concise way,

$$
C_{1}(r)=\frac{d}{d r}[r C(r)]
$$

In practice, given the covariance function $C(\cdot)$ in domain $\Omega$, we can use Eq. (2.16) to derive the covariance function on each line. 
Mantoglou [81] used the spectral approach to derive the relation of power spectral density of the original 3-D process and the corresponding line process,

$$
S_{\phi, \theta}^{(1)}=2 \pi k S(k \sin \phi \cos \theta, k \sin \phi \sin \theta, k \cos \phi) .
$$

In the 2-D case, results are more complicated. In polar coordinate $(r, \theta)$, set $y$-axis to be $h=x_{2}-x_{1}$ direction, as can be seen in Fig. 4 . Then,

$$
C(r)=\frac{1}{2 \pi} \int_{0}^{2 \pi} C_{1}(r \sin \theta) d \theta .
$$

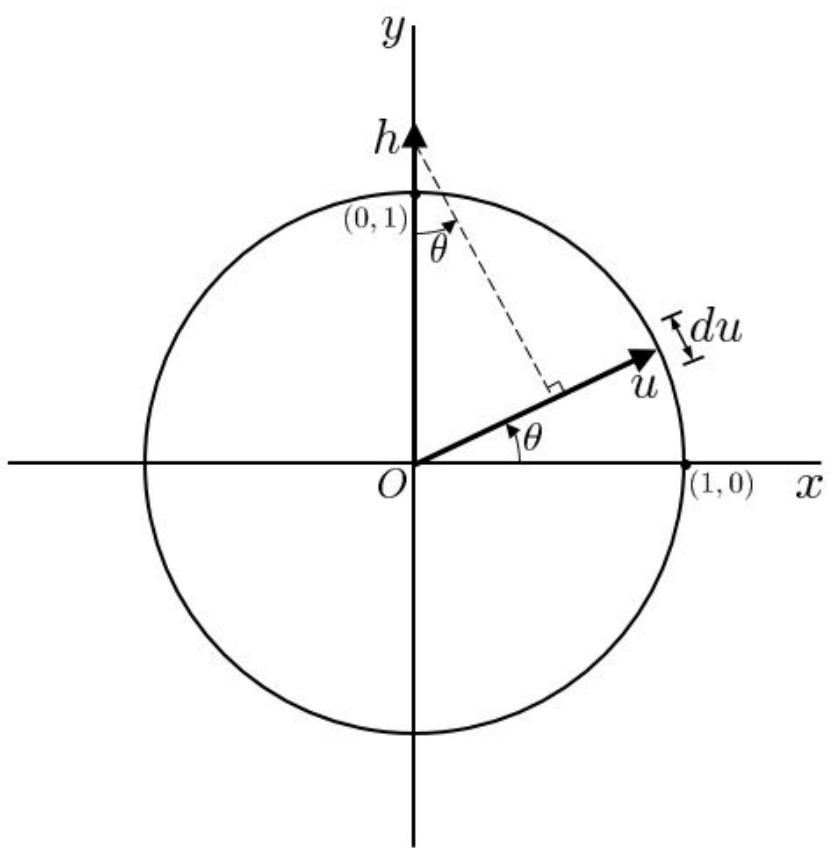

Fig. 4 Integration in unit circle, Mantoglou and Wilson (1982) [83]

Substituting $\zeta=r \sin \theta$ into Eq. (2.18) and also exploiting the symmetric property of the integration, we obtain,

$$
\begin{aligned}
C(r) & =\frac{2}{\pi} \int_{0}^{\frac{\pi}{2}} C_{1}(\zeta) d\left(\arcsin \frac{\zeta}{r}\right) \\
& =\frac{2}{\pi} \int_{0}^{r} \frac{1}{r} \frac{C_{1}(\zeta)}{\sqrt{1-\frac{\zeta^{2}}{r^{2}}}} d(\zeta) \\
& =\frac{2}{\pi} \int_{0}^{r} \frac{C_{1}(\zeta)}{\sqrt{r^{2}-\zeta^{2}}} d(\zeta) .
\end{aligned}
$$


However, in most cases Eq. (2.19) is impractical to derive a closed form formula of $C_{1}(\zeta)$. To circumvent this problem, the power spectral density method was applied.

Definition 1 (Power Spectral Density) The power spectral density $S(\boldsymbol{\omega})$ of a random process $K$ with covariance function $C(\boldsymbol{h})$ is defined as,

$$
S(\boldsymbol{\omega})=\frac{1}{(2 \pi)^{n}} \int_{\mathbb{R}^{n}} e^{-i \boldsymbol{h} \cdot \boldsymbol{\omega}} C(\boldsymbol{h}) d \boldsymbol{h} .
$$

Conversely,

$$
C(\boldsymbol{h})=\int_{\mathbb{R}^{n}} e^{i \boldsymbol{h} \cdot \boldsymbol{\omega}} S(\boldsymbol{\omega}) d \boldsymbol{\omega} .
$$

The above Fourier transform pair is often referred to as Wiener-Khinchin formula. In our case, the random field is assumed to be isotropic, then the vector $\boldsymbol{h}$ and $\boldsymbol{\omega}$ can be replaced by its magnitude $|\boldsymbol{h}|$ and $|\boldsymbol{\omega}|$ respectively.

In the 2-D case, the power spectral density function can be expressed in the sense of polar coordinates as follows, which yields a radial power spectrum.

Definition 2 (Radial Power Spectral Density) The radial power spectral density is defined by,

$$
R(\omega)=\frac{1}{\sigma^{2}} \int_{\partial B(0, \omega)} S(\omega \cos \theta, \omega \sin \theta) d \theta .
$$

In our case, the random field is isotropic, then the radial power spectral density becomes,

$$
R(\omega)=\frac{2 \pi \omega}{\sigma^{2}} S(\omega)
$$

Now we take advantage of the isotropic property and transform Eq. (2.21) into polar coordinates $(r, \theta)$, and set the azimuth direction orthogonal to $h=$ $x_{2}-x_{1}$ direction,

$$
\begin{aligned}
C(\boldsymbol{h}) & =\int_{R^{2}} e^{i \boldsymbol{h} \cdot \boldsymbol{\omega}} S(\boldsymbol{\omega}) d \boldsymbol{\omega} \\
& =\int_{0}^{2 \pi} \int_{0}^{\infty} e^{i h r \sin \theta} S(r) r d r d \theta \\
& =\int_{0}^{\infty} \int_{0}^{2 \pi} \cos (r h \sin \theta) d \theta r S(r) d r \\
& =\int_{0}^{\infty} 2 \pi J_{0}(h r) r S(r) d r \\
& =\sigma^{2} \int_{0}^{\infty} J_{0}(h r) R(r) d r .
\end{aligned}
$$

By Hankel transform we can obtain,

$$
R(r)=\frac{r}{\sigma^{2}} \int_{0}^{\infty} C(h) J_{0}(h r) h d h .
$$


For 1-D line process with the covariance function $C_{1}(\zeta)$, its equivalence to Eq. (2.20) is

$$
C_{1}(\zeta)=\int_{-\infty}^{+\infty} e^{i \omega \zeta} S_{1}(\omega) d \omega=2 \int_{0}^{+\infty} \cos (\omega \zeta) S_{1}(\omega) d \omega
$$

Substituting Eq. (2.26) into integration Eq. (2.19) and changing the integration order yields,

$$
\begin{aligned}
C(r) & =\frac{2}{\pi} \int_{0}^{r} \frac{C_{1}(\zeta)}{\sqrt{r^{2}-\zeta^{2}}} d \zeta \\
& =\frac{4}{\pi} \int_{0}^{r} \frac{\int_{0}^{\infty} \cos (\omega \zeta) S_{1}(\omega) d \omega}{\sqrt{r^{2}-\zeta^{2}}} d \zeta \\
& =\frac{4}{\pi} \int_{0}^{r} \frac{\int_{0}^{\infty} \cos (\omega r \cos \theta) S_{1}(\omega) d \omega}{r \sin \theta} r \sin \theta d \theta \\
& =\frac{4}{\pi} \int_{0}^{\infty} S_{1}(\omega)\left[\int_{0}^{\frac{\pi}{2}} \cos (\omega r \cos \theta) d \theta\right] d \omega .
\end{aligned}
$$

It should be noticed that,

$$
\int_{0}^{\frac{\pi}{2}} \cos (\omega r \cos \theta) d \theta=\frac{\pi}{2} J_{0}(\omega r)
$$

where $J_{0}(\cdot)$ is a Bessel function of first kind of order zero. Then,

$$
C(r)=2 \int_{0}^{\infty} S_{1}(\omega) J_{0}(\omega r) d \omega
$$

Apply a Hankel transform we obtain,

$$
S_{1}(\omega)=\frac{1}{2} \omega \int_{0}^{\infty} C(r) J_{0}(\omega r) r d r
$$

Compare Eq. (2.30) and Eq. (2.25) the following relation can be derived,

$$
S_{1}(\omega)=\frac{\sigma^{2}}{2} R(\omega)
$$

When the radical spectral density function $f(\omega)$ of 2-D isotropic random process is obtained, the spectral density function $S_{1}(\omega)$ of the corresponding line process can be computed, and from the spectral density a line process can be generated. 


\subsubsection{Line Process Generation}

In general, the line process generation methods that appear next will inevitably overlap with the following subsections, since they methods of random field generation as well. In the following we will mainly discuss the line process generation method that has frequently appeared in the context of TBM.

Shinozuka $[114,116]$ proposed that a random process can be represented as summation of a series of cosine functions with random frequency. A $d$ dimensional Gaussian random process $f(\boldsymbol{x})$ with the spectral density function $S(\boldsymbol{\omega})$ can be expressed as,

$$
f(\boldsymbol{x})=\sqrt{\frac{2}{N}} \sigma \sum_{k=1}^{N} \cos \left(\boldsymbol{\omega}_{\boldsymbol{k}} \cdot \boldsymbol{x}+\phi_{k}\right),
$$

where $\phi_{k} \sim U[0,2 \pi], \boldsymbol{\omega}_{\boldsymbol{k}}$ are sampled according to the joint density function $g$,

$$
g(\boldsymbol{\omega})=S(\boldsymbol{\omega}) / \sigma^{2},
$$

$\sigma^{2}$ is the normalizing factor,

$$
\sigma^{2}=\int_{\mathbb{R}^{d}} S(\boldsymbol{\omega}) d \boldsymbol{\omega}
$$

Mantoglou [83] applied the spectral approach on the generation of line process. Suppose that a line random process $f_{0}(x)$ has the autocovariance function $R_{0}(\xi)$, which is given by,

$$
R_{0}(\xi)=\mathbb{E}\left[f_{0}(x) \cdot f_{0}(x+\xi)\right] .
$$

Notice that $R_{0}(\xi)$ is an even function, $R_{0}(\xi)=R_{0}(-\xi)$. Then the spectral density function $S_{0}(\omega)$ is given by,

$$
S_{0}(\omega)=\frac{1}{2 \pi} \int_{-\infty}^{+\infty} R_{0}(\xi) e^{-i \omega \xi} d \xi=\frac{1}{2 \pi} \int_{-\infty}^{+\infty} R_{0}(\xi) \cos (\omega \xi) d \xi
$$

Now select an interval $\left[\omega_{l}, \omega_{u}\right]$ in which the significant magnitude of frequencies are contained. Divide this interval into $N$ subintervals with equal length, and pick the $\omega^{\prime} s$ at each center of the intervals, i.e.,

$$
\begin{aligned}
\Delta \omega & =\frac{\omega_{u}-\omega_{l}}{N}, \\
\omega_{k} & =\omega_{l}+\left(k-\frac{1}{2}\right) \Delta \omega, \quad k=1,2, \cdots, N .
\end{aligned}
$$

Then the random process can be generated by,

$$
f(x)=\sqrt{2} \sum_{k=1}^{N}\left(S_{0}\left(\omega_{k}\right) \Delta \omega\right)^{\frac{1}{2}} \cos \left(\omega_{k}^{\prime} \cdot x+\phi_{k}\right),
$$


where

$$
\begin{gathered}
\phi_{k} \stackrel{i . i . d .}{\sim} U[0,2 \pi], \\
\omega_{k}^{\prime}=\omega_{k}+\delta \omega_{k},
\end{gathered}
$$

with $\delta \omega_{k} \sim U\left[-\Delta \omega^{\prime} / 2, \Delta \omega^{\prime} / 2\right]$ and $\Delta \omega^{\prime} \ll \Delta \omega$. $\delta \omega_{k}$ is introduced to avoid periodicity.

It is not complicated to show that $\mathbb{E}[f(x)]=0$. Now we focus on the autocorrelation function $R(\xi)$,

$$
\begin{aligned}
R(\xi)= & \mathbb{E}[f(x) f(x+\xi)] \\
= & \mathbb{E}\left[\sqrt{2} \sum_{k=1}^{N}\left(S_{0}\left(\omega_{k}\right) \Delta \omega\right)^{\frac{1}{2}} \cos \left(\omega_{k}^{\prime} \cdot x+\phi_{k}\right)\right. \\
& \left.\cdot \sqrt{2} \sum_{k=1}^{N}\left(S_{0}\left(\omega_{k}\right) \Delta \omega\right)^{\frac{1}{2}} \cos \left(\omega_{k}^{\prime} \cdot(x+\xi)+\phi_{k}\right)\right] \\
= & \sum_{k=1}^{N} 2 S_{0}\left(\omega_{k}\right) \Delta \omega \cdot \mathbb{E}\left[\cos \left(\omega_{k}^{\prime} \cdot x+\phi_{k}\right) \cdot \cos \left(\omega_{k}^{\prime} \cdot(x+\xi)+\phi_{k}\right)\right] \\
= & \sum_{k=1}^{N} S_{0}\left(\omega_{k}\right) \Delta \omega \cdot \mathbb{E}\left[\cos \left(\omega_{k}^{\prime} \xi\right)\right] .
\end{aligned}
$$

Since $\Delta \omega^{\prime} \ll \Delta \omega$, the following approximation can be accepted,

$$
\mathbb{E}\left[\cos \left(\omega_{k}^{\prime} \xi\right)\right] \approx \cos \left(\omega_{k} \xi\right)
$$

As $k \rightarrow \infty$, Eq. (2.41) becomes,

$$
\begin{aligned}
R(\xi) & =\int_{-\infty}^{+\infty} S_{0}(\omega) \cos (\omega \xi) d \omega \\
& =\int_{-\infty}^{+\infty} S_{0}(\omega)(\cos (\omega \xi)+i \sin (\omega \xi)) d \omega \\
& =R_{0}(\xi)
\end{aligned}
$$

Thus the covariance structure of the line process is preserved.

Tompson [127] applied the Fast-Fourier transform (FFT) to generate line process. Any zero-mean, real-valued random process $Z(\zeta)$ can be represented as the real part of a complex random process $X(\zeta)$ [105],

$$
X(\zeta)=Z(\zeta)+i Y(\zeta)
$$

while the complex random process $X(\zeta)$ can be represented by a FourierStieltjes transformation,

$$
X(\zeta)=\int e^{i k \zeta} d W(k) \approx \sum_{\Delta k} e^{i k_{j} \zeta} d W\left(k_{j}\right) .
$$


The Fourier increments $d W$ satisfies the following relations,

$$
\begin{aligned}
\mathbb{E}[d W(k)] & =0, \\
\mathbb{E}\left[d W\left(k_{1}\right) d W^{*}\left(k_{2}\right)\right] & =0, \quad \forall k_{1} \neq k_{2}, \\
\mathbb{E}\left[|d W(k)|^{2}\right] & =2 S_{1}(k) d k .
\end{aligned}
$$

Let $f=k / 2 \pi$ and $d W\left(k_{j}\right)=d W\left(f_{j}\right)$. Divide the simulation domain by $M$ equispaced points, with $\zeta_{n}=n \Delta \zeta$ and $f_{j}=j \Delta f$, where $j, n=0,1, \cdots, M-1$. For FFT works, we also need that,

$$
\Delta f \Delta \zeta=1 / M
$$

Then,

$$
X\left(\zeta_{n}\right)=\int e^{i 2 \pi f \zeta_{n}} d W(f) \approx \sum_{j=0}^{M-1} e^{i 2 \pi j n / M} d W\left(f_{j}\right)
$$

Let $\omega=e^{2 \pi i / M}$, and denote $X\left(\zeta_{n}\right), d W\left(f_{j}\right)$ as $X_{n}$ and $d W_{j}$ respectively, the Eq. (2.48) can be written in matrix form,

$$
\left[\begin{array}{c}
X_{0} \\
X_{1} \\
X_{2} \\
\cdots \\
X_{M-1}
\end{array}\right]=\left[\begin{array}{cccccc}
1 & 1 & 1 & 1 & \cdots & 1 \\
1 & \omega & \omega^{2} & \omega^{3} & \cdots & \omega^{M-1} \\
1 & \omega^{2} & \omega^{4} & \omega^{6} & \cdots & \omega^{2(M-1)} \\
\cdots & \cdots & \cdots & \cdots & \cdots & \cdots \\
1 & \omega^{M-1} & \omega^{2(M-1)} & \omega^{3(M-1)} & \cdots & \omega^{(M-1)(M-1)}
\end{array}\right] \cdot\left[\begin{array}{c}
d W_{0} \\
d W_{1} \\
d W_{2} \\
\cdots \\
d W_{M-1}
\end{array}\right] .
$$

FFT exploits the periodicity of complex exponential, notice that the summation in Eq. (2.48) can be splitted into even-indexed terms and odd-indexed terms,

$$
\begin{aligned}
X_{k} & =\sum_{j=0}^{M / 2-1} d W_{2 j} e^{\frac{2 \pi i}{M} 2 j k}+\sum_{j=0}^{M / 2-1} d W_{2 j+1} e^{\frac{2 \pi i}{M}(2 j+1) k} \\
& =\sum_{j=0}^{M / 2-1} d W_{2 j} e^{\frac{2 \pi i}{M / 2} j k}+e^{\frac{2 \pi i k}{N}} \cdot \sum_{j=0}^{M / 2-1} d W_{2 j+1} e^{\frac{2 \pi i}{M / 2} j k} \\
& =E_{k}+e^{\frac{2 \pi i k}{N}} O_{k} .
\end{aligned}
$$

It should be noticed that $E_{k}$ and $O_{k}$ are the discrete Fourier transforms of even-indexed and odd-indexed terms respectively.

The term $X_{k+M / 2}$ can be obtained from,

$$
\begin{aligned}
X_{k+M / 2} & =\sum_{j=0}^{M / 2-1} d W_{2 j} e^{\frac{2 \pi i}{M / 2} j\left(k+\frac{M}{2}\right)}+e^{\frac{2 \pi i\left(k+\frac{M}{2}\right)}{N}} \cdot \sum_{j=0}^{M / 2-1} d W_{2 j+1} e^{\frac{2 \pi i}{M / 2} j\left(k+\frac{M}{2}\right)} \\
& =\sum_{j=0}^{M / 2-1} d W_{2 j} e^{\frac{2 \pi i}{M / 2} j k}-e^{\frac{2 \pi i k}{M}} \sum_{j=0}^{M / 2-1} d W_{2 j+1} e^{\frac{2 \pi i}{M / 2} j k} \\
& =E_{k}-e^{\frac{2 \pi i k}{M}} O_{k} .
\end{aligned}
$$


The $E_{k}$ and $O_{k}$ computed for $X_{k}$ can also be used in the computation for $X_{k+M / 2}$. Notice that the computation of $E_{k}$ and $O_{k}$ can be splitted into even and odd part as well. The FFT method recursively divides the number of elements in the Fourier transform, thus reduces the complexity from $\mathcal{O}\left(M^{2}\right)$ to $\mathcal{O}(M \log M)$. In FFT case, $M, \Delta f, \Delta \zeta, f_{\max }, \zeta_{\max }$ must be chosen carefully, since any two parameters can determine the remaining three.

After that we have generated a sample of $X$, and it can be proved that its real part $Z$ is identically distributed as imaginary part $Y$. Therefore we have generated two realizations of original random field $K$.

Hunger [53,54] applied the Halton sequence instead of uniformly distributed random sequence to generate line orientations. The algorithm was implemented on GPU. Emery [28] employed TBM to generate conditional data.

TBM has the merit of low computational cost, however, the simulation generated by such method is not exact. The error has two sources. First, practically we are only able to generate finite number of lines. Although previous results show that evenly spaced lines in 2-D [82], or the use of low-discrepancy sequence $[53,54]$ can accelerate the convergence rate, the statistical error still exist. Second, the continuous process is approximated by discrete bands within each line. For each point in space, we can generate the projection onto each line accurately, but the cost will be prohibitive compared to the discrete bands.

\subsection{Spectral Method}

Spectral method generates the random field by the manipulation in the frequency domain, and we have already mentioned spectral method in 1-D in Subsection 2.2.2. The method can be traced back to $[114,116,86]$. Many works $[104,96,46,105,65,107]$ involve the generation of multiple correlated random fields, for simplicity, in this review we only present the generation of a single real-valued random field.

Recall the definition of power spectral density,

$$
S(\boldsymbol{k})=\int_{-\infty}^{+\infty} e^{-2 \pi i \boldsymbol{k} \cdot \boldsymbol{x}} C(\boldsymbol{x}) d \boldsymbol{x} .
$$

A general complex-valued random field $K(\cdot)$ can be expressed as a FourierStieltjes integral over the Fourier increment $d W$,

$$
K(\boldsymbol{x})=\int_{-\infty}^{+\infty} e^{i \boldsymbol{k} \cdot \boldsymbol{x}} d W(\boldsymbol{k})
$$

where the fourier increment $d W$ satisfies,

$$
\begin{aligned}
\mathbb{E}[d W(\boldsymbol{k})] & =0, \\
\mathbb{E}\left[d W(\boldsymbol{k}) \cdot d W^{*}\left(\boldsymbol{k}^{\prime}\right)\right] & =2 S(\boldsymbol{k}) d \boldsymbol{k} \cdot \delta\left(\boldsymbol{k}-\boldsymbol{k}^{\prime}\right) .
\end{aligned}
$$




\subsubsection{Fourier Transform}

First, decompose $S, \forall \boldsymbol{k} \in \mathbb{R}^{n}$,

$$
S(\boldsymbol{k})=H(\boldsymbol{k}) \cdot H^{*}(\boldsymbol{k}),
$$

the random Fourier increment is then given by,

$$
d W(\boldsymbol{k}) \approx \Delta W(\boldsymbol{k})=H(\boldsymbol{k}) e^{i \theta(\boldsymbol{k})}|\Delta \boldsymbol{k}|^{1 / 2},
$$

where $\theta(\boldsymbol{k}) \stackrel{\text { i.i.d. }}{\sim} U[0,2 \pi]$. Eq. (2.56) can be justified, since,

$$
\begin{aligned}
\mathbb{E}[\Delta W(\boldsymbol{k})] & =H(\boldsymbol{k}) \mathbb{E}\left[e^{i \theta(\boldsymbol{k})}\right]|\Delta \boldsymbol{k}|^{1 / 2}=0, \\
\mathbb{E}\left[\Delta W(\boldsymbol{k}) \Delta W^{*}\left(\boldsymbol{k}^{\prime}\right)\right] & =S(\boldsymbol{k}) \Delta \boldsymbol{k} \mathbb{E}\left[e^{i \theta(\boldsymbol{k})-i \theta\left(\boldsymbol{k}^{\prime}\right)}\right]=S(\boldsymbol{k}) \Delta \boldsymbol{k} \cdot \delta\left(\boldsymbol{k}-\boldsymbol{k}^{\prime}\right) .
\end{aligned}
$$

Then the value at a point $\boldsymbol{x}$ is given by,

$$
K(\boldsymbol{x})=\sum_{-\boldsymbol{B}}^{\boldsymbol{B}} e^{i \boldsymbol{k} \cdot \boldsymbol{x}} \Delta W(\boldsymbol{k}) .
$$

where $[-\boldsymbol{B}, \boldsymbol{B}]$ is the bandwidth.

It should be noticed that in the above derivation, $\boldsymbol{k}$ is generally a vector depending on the dimension of the random field. The complex-valued random field $K(\boldsymbol{x})$ can be expressed as $K(\boldsymbol{x})=Y(\boldsymbol{x})+i Z(\boldsymbol{x})$, and it can be proved [105] there is no correlation between the real part $Y$ and the complex part $Z$. Thus by now two realizations of the random field have been generated.

\subsubsection{Fast Fourier Transform}

The integration in Eq. (2.53) can also be conducted in Fast Fourier Transform(FFT) way. Lang [65] analyzed complexities of FFT, note that in FFT setting the spatial location and frequency discretization have to satisfy following certain conditions,

$$
\begin{gathered}
x_{m_{j}}=m_{j} \Delta x_{j}, \quad m_{j}=1, \cdots, L_{j}, \\
k_{n_{j}}=n_{j} \Delta k_{j}, \quad n_{j}=1, \cdots, L_{j}, \\
\Delta k_{j}=\frac{2 \pi}{L_{j} \Delta x_{j}} .
\end{gathered}
$$

In Eq. (2.59), $x_{m_{j}}$ is the location of $m_{t h}$ point in $j_{t h}$ dimension, $k_{n_{j}}$ is the $n_{t h}$ wave number in $j_{t h}$ dimension. $\Delta x_{j}$ and $\Delta k_{j}$ are increments in $j_{t h}$ dimension of location and wave number respectively. $L_{j}$ is the total number of discretization in $j_{t h}$ dimension. Eq. (2.60) states the relation that $\Delta k_{j}$ and $\Delta x_{j}$ must satisfy.

In the 2-D case, Eq. (2.58) becomes,

$$
K(j, l)=\sum_{n=0}^{L_{2}-1} \sum_{m=0}^{L_{1}-1} \Delta\left(m \Delta k_{1}, n \Delta k_{2}\right) e^{i m j \frac{2 \pi}{L_{1}}} e^{i n l \frac{2 \pi}{L_{2}}} .
$$


This can be viewed as two fast Fourier transforms. In matrix form,

$$
K=W_{L_{1}} \Delta W_{L_{2}}
$$

where $K, \Delta \in \mathbb{R}^{L_{1} \times L_{2}}, W_{L_{1}} \in \mathbb{R}^{L_{1} \times L_{1}}$ and $W_{L_{2}} \in \mathbb{R}^{L_{2} \times L_{2}}$ are Fourier transform matrices. For detailed implementation, readers can refer to $[46,105]$.

\subsection{Matrix Decomposition Method}

Matrix decomposition method is an easy-to-implement approach to generate the random field. This method was first proposed in [17]. Suppose we are only interested in $N$ points in the random field, which are denoted as a vector $\boldsymbol{x} \in \mathbb{R}^{N}$, then a covariance matrix $R$ involving those points can be constructed. Since the covariance matrix $R$ is symmetric positive-definite, the Cholesky factorization can be applied as,

$$
R=L U
$$

where $L^{T}=U$. Generate a standard Gaussian random vector $\xi \in \mathbb{R}^{N}$, i.e. $\mathbb{E}\left[\xi_{i}\right]=0, \mathbb{E}\left[\xi_{i} \xi_{j}\right]=\delta_{i j}$, for $i, j=1, \cdots, N$, then the values at the points $\boldsymbol{x}$ are given by,

$$
K(\boldsymbol{x})=L \xi
$$

This can be easily verified since the mean and covariance are preserved,

$$
\begin{aligned}
\mathbb{E}[K(\boldsymbol{x})] & =0, \\
\mathbb{E}\left[K(\boldsymbol{x}) K^{T}(\boldsymbol{x})\right] & =L \mathbb{E}\left[\xi \xi^{T}\right] U=R .
\end{aligned}
$$

Straightforward as it is, the method suffers from several deficiencies. First, the size of the covariance matrix is $N \times N$, with the number of nodes increasing, the program is prone to run out of memory. Unfortunately in real-world groundwater modeling, the degree of freedom is typically rather large. Second, the cost of Cholesky decomposition of the covariance matrix is $\mathcal{O}\left(N^{3}\right)$, which becomes computationally intractable for large $N$. Third, the matrix-vector multiplication to generate the random field $K$ also costs $\mathcal{O}\left(N^{2}\right)$. To reduce the computational complexity, multiple approaches were proposed, these including polynomial approximation of $R^{1 / 2}$ [19], circulant embedding and $\mathcal{H}$-Matrix approximation. Here we briefly introduce the latter two methods.

\subsubsection{Circulant Embedding}

Circulant embedding method for covariance matrix decomposition was proposed by Dietrich [20], recent developments are in [40,97]. For simplicity, in the following we introduce the 1-D case. 
In 1-D situation, suppose equispaced points $x_{0}, x_{1}, \cdots, x_{m}$, and denote the covariance function by $\rho_{|i-j|}=C\left(x_{i}, x_{j}\right)$. Then the covariance matrix $R$ can be written as,

$$
R=\left[\begin{array}{ccccccc}
\rho_{0} & \rho_{1} & \rho_{2} & \rho_{3} & \cdots & \rho_{m-1} & \rho_{m} \\
\rho_{1} & \rho_{0} & \rho_{1} & \rho_{2} & \cdots & \rho_{m-2} & \rho_{m-1} \\
\rho_{2} & \rho_{1} & \rho_{0} & \rho_{1} & \cdots & \rho_{m-3} & \rho_{m-2} \\
\cdots & \cdots & \cdots & \cdots & \cdots & \cdots & \cdots \\
\rho_{m} & \rho_{m-1} & \rho_{m-2} & \rho_{m-3} & \cdots & \rho_{1} & \rho_{0}
\end{array}\right]
$$

Matrix $R$ is symmetric (block) Toeplitz matrix, and it can be denoted as,

$$
R=S B T\left(\rho_{0}, \rho_{1}, \cdots, \rho_{m}\right) .
$$

Now extend the matrix $R$ to a matrix $S, S \in \mathbb{R}^{2 M \times 2 M}$

$$
S=S B T\left(\rho_{0}, \rho_{1}, \cdots, \rho_{m}, \rho_{m+1}, \rho_{m+2}, \cdots, \rho_{2 M-m}, \cdots, \rho_{2 M-1}\right),
$$

where $\rho_{2 M-k}=\rho_{k}$, for $k=1, \cdots, m-1$, and the values of $\rho_{m+1}, \rho_{m+2}, \cdots$, $\rho_{2 M-m}$ are chosen arbitrarily. The introduction of these values is called "padding" and it ensures the extended matrix $S$ is positive-definite. Then $S$ has an eigenvalue decomposition,

$$
S=\frac{1}{2 M} F \Lambda F^{H},
$$

where $F$ is the standard FFT matrix with elements $F_{p q}=\exp (2 \pi i p q / 2 M)$. $\Lambda=\operatorname{diag}(\boldsymbol{\lambda})$, where $\boldsymbol{\lambda}$ is the discrete Fourier transform of the first row of $S$, i.e., $\boldsymbol{\lambda}=F S_{1}^{T}$. Let $\boldsymbol{\epsilon}=\boldsymbol{\alpha}+i \boldsymbol{\beta}$ be a complex random vector where $\mathbb{E}[\boldsymbol{\alpha}]=0$, $\mathbb{E}[\boldsymbol{\beta}]=0, \mathbb{E}\left[\boldsymbol{\alpha} \boldsymbol{\alpha}^{T}\right]=I, \mathbb{E}\left[\boldsymbol{\beta} \boldsymbol{\beta}^{T}\right]=I, \mathbb{E}\left[\boldsymbol{\alpha} \boldsymbol{\beta}^{T}\right]=0$. Then a realization $K \in \mathbb{C}^{2 M}$ can be generated by,

$$
K=F(\Lambda / 2 M)^{1 / 2} \boldsymbol{\epsilon},
$$

It can be easily shown that,

$$
\begin{aligned}
\mathbb{E}\left[K K^{*}\right] & =2 S, \\
\mathbb{E}\left[K K^{T}\right] & =0, \\
\mathbb{E}\left[\operatorname{Re}(K) \cdot \operatorname{Re}(K)^{T}\right] & =S, \\
\mathbb{E}\left[\operatorname{Im}(K) \cdot \operatorname{Im}(K)^{T}\right] & =S .
\end{aligned}
$$

This indicates that both the real and imaginary parts of $K$ is distributed as $N(0, S)$. Notice that, any $(m+1) \times(m+1)$ block along the diagonal of $S$ is exactly the matrix $R$. Then the random vector of interest can be determined by selecting any consecutive $(m+1)$ entries in $\operatorname{Re}(K)$ or $\operatorname{Im}(K)$.

Now consider the $2-\mathrm{D}$ case. The random field $K(x, y)$ has a covariance function $C(x, y)$, the grid is rectangular mesh with $(m+1) \times(n+1)$ points and $\Delta x, \Delta y$ being interval in respective direction. We denote $\rho_{j, i}=C(i \Delta x, j \Delta y)$, then the covariance matrix $R$ is symmetric block toeplitz,

$$
R=S B T\left\{B_{0}, B_{1}, B_{2}, \cdots, B_{n}\right\},
$$


with each block $B_{k} \in \mathbb{R}^{(m+1) \times(m+1)}$ symmetric toeplitz matrix, $k=0,1, \cdots, n$

$$
B_{k}=S B T\left\{\rho_{k, 0}, \rho_{k, 1}, \rho_{k, 2}, \cdots, \rho_{k, m}\right\} .
$$

To extend the matrix $R$, the first step is to extend each block $B_{k}$ to circulant matrix $\bar{B}_{k}$ as in the $1-\mathrm{D}$ case. Now the matrix becomes,

$$
R^{\prime}=S B T\left\{\bar{B}_{0}, \bar{B}_{1}, \bar{B}_{2}, \cdots, \bar{B}_{n}\right\} .
$$

The second step is to extend $R^{\prime}$ to a block circulant matrix,

$$
S=S B T\left\{\bar{B}_{0}, \bar{B}_{1}, \bar{B}_{2}, \cdots, \bar{B}_{n}, \bar{B}_{n-1}, \cdots, \bar{B}_{1}\right\} .
$$

During the two steps, add padding if necessary. Then $S \in \mathbb{R}^{d \times d}$ is circulant matrix and has the following spectral decomposition,

$$
S=\frac{1}{d} F \Lambda F^{H} .
$$

Then we can have $K$ given by $K=\frac{1}{\sqrt{d}} F \Lambda^{1 / 2} \boldsymbol{\epsilon}$, where $\boldsymbol{\epsilon}$ is defined same as above, and $\operatorname{Re}(K), \operatorname{Im}(K) \sim N(0, S)$. Since $R$ is embedded in $S$, the corresponding entries in $K$ can be extracted and thus this is a realization of the random field.

The improvement in circulant embedding comes from the FFT in computing $\Lambda^{1 / 2}$ and the matrix-vector multiplication $F \Lambda^{1 / 2} \epsilon$. It reduces the computational complexity from $\mathcal{O}\left(m^{3}\right)$ to $\mathcal{O}(m \log m)$. However, the limitation of this method is that points must be set on rectangular grid.

\subsubsection{H-Matrix}

$\mathcal{H}$-Matrix was introduced in [49], the application of $\mathcal{H}$-Matrix in the context of covariance matrix is quite recent $[29,10]$. The essence of $\mathcal{H}$-Matrix is to approximate the covariance matrix $R$ by an $\mathcal{H}$-matrix, then obtain $R^{1 / 2}$ within $\mathcal{H}$-Matrix algorithm. Afterwards the random field can be generated by the matrix-vector multiplication $R^{1 / 2} \xi$. Fig. 5 provides an example of $\mathcal{H}$-Matrix.

Briefly speaking, the construction of $\mathcal{H}$-matrix consists of two steps. Firstly, divide the matrix into near-field blocks and far-field blocks. Secondly, store the values in the near-field blocks exactly but approximate each far-field block with low-rank matrix.

The following briefly introduces the construction of $\mathcal{H}$-Matrix. Denote $N=$ $\left\{x_{1}, x_{2}, \cdots x_{N}\right\}$ as the set of all points. For each subset $X \subseteq N$ we denote $B_{X} \subseteq \mathbb{R}^{d}$, which is the smallest axis-aligned bounding box such that $X \subseteq B_{X}$. Let the root of the cluster tree be the set of whole points, i.e., $X_{\text {root }}=N$, split $B_{X}$ along its longest edge into $B_{0}$ and $B_{1}$ such that $B_{0} \bigcup B_{1}=B_{X}$, see Fig. 6a. Then the son of $X$, sons $(X)=\left\{X_{0}, X_{1}\right\}$, are $X_{0}=X \cap B_{0}$, $X_{1}=X \bigcap B_{1}$. Keep dividing the box until the size reaches a threshold. After the whole process a binary tree is constructed, which is also called cluster tree. 


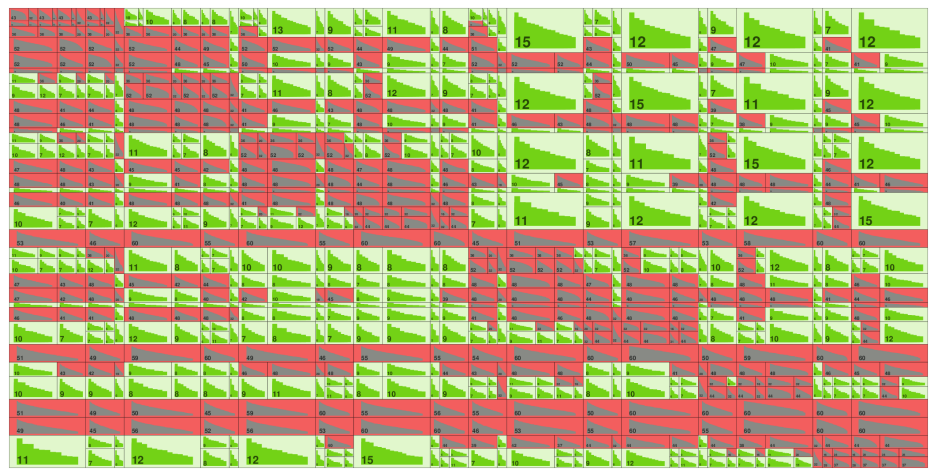

Fig. 5 An example of Hierarchical matrix generated by Hlibpro(https://www.hlibpro.com/). The red blocks are exact data storage, while the green blocks are low rank approximation. The number in each block represents rank

Suppose $B$ and $B^{\prime}$ are the nodes from a cluster tree. Then admissibility condition (see Fig. 6b) for axis-aligned bounding box $B$ and $B^{\prime}$ is given by,

$$
\max \left\{\operatorname{diam}(B), \operatorname{diam}\left(B^{\prime}\right)\right\} \leq \eta \cdot \operatorname{dist}\left(B, B^{\prime}\right),
$$

where $\operatorname{dist}\left(B, B^{\prime}\right)=\inf _{x \in B, y \in B^{\prime}}|x-y|$ and $\eta \in \mathbb{R}$ determines the level of admissibility. If the above admissibility condition is satisfied, denote that $\operatorname{adm}\left(B, B^{\prime}\right)=$ true. Under this condition the approximation error is exponentially convergent $[49]$.

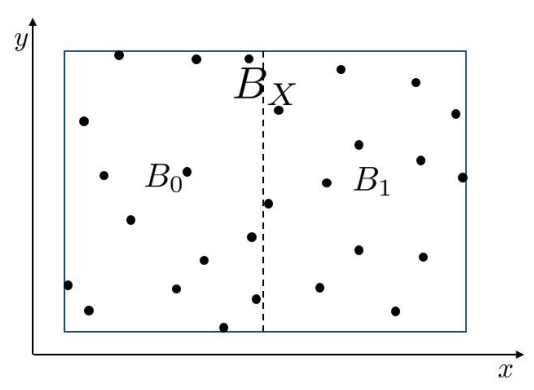

(a) Axis-aligned bounding box

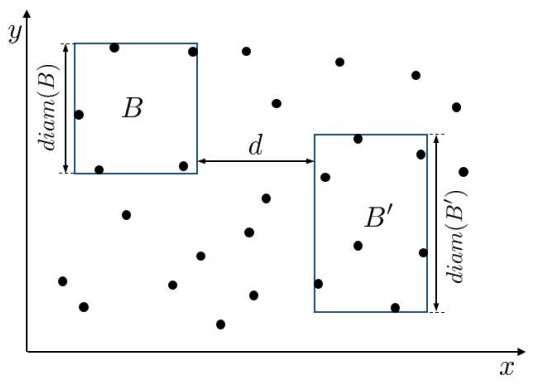

(b) Admissbility condition

Fig. 6 Illustration of constructing the block cluster tree

Then the block cluster tree can be built, whose name indicates that each node in such tree corresponds to a block in the covariance matrix. The root of the tree is $X_{\text {root }} \times X_{\text {root }}$, which corresponds with the whole covariance matrix $C$. For a node in such tree $X \times Y$, their sons have the following possible situations, 


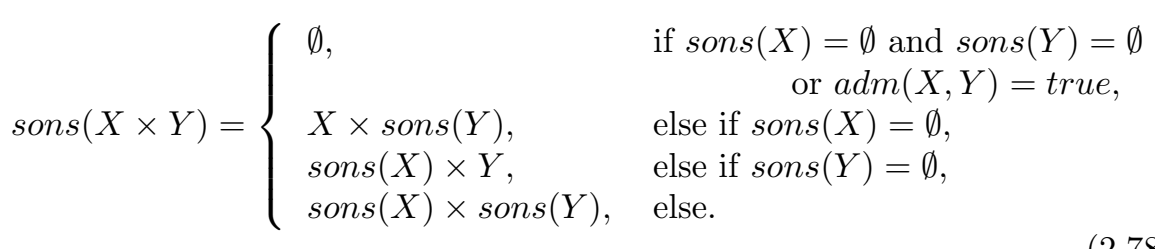

Based on the above procedure, the block cluster tree can be constructed. Notice that leaf nodes form a partition of the covariance matrix $C$. The admissible blocks can be approximated by the low rank matrix, other blocks store the exact information.

Compared with the direct matrix decomposition method, $\mathcal{H}$-matrix algorithm can reduce the computational complexity. Moreover, this method does not require points ordered in rectangular mesh nor stationary covariance function.

\subsection{Karhunen-Loève Expansion}

\subsubsection{General Formulation}

Karhunen-Loève expansion (KL-expansion)[79] is an approach to decompose a random process into the eigenvalues $\lambda$ and eigenfunctions $f$ of its covariance kernel. The method is frequently involved in modeling random fields, see $[36,136,111,71,68,139]$ for example.

Since the covariance kernel is bounded, symmetric positive definite and thus it can be decomposed as,

$$
C(x, y)=\sum_{n=1}^{\infty} \lambda_{n} f_{n}(x) f_{n}(y),
$$

where the eigenvalues and eigenfunctions are given by the following Fredholm integral equation,

$$
\int_{\Omega} C(x, y) f(x) d x=\lambda f(y) .
$$

Thus samples of $K$ are given by,

$$
K(x, \omega)=\sum_{i=1}^{\infty} \sqrt{\lambda_{i}} f_{i}(x) \xi_{i}(\omega),
$$

where the random variables $\xi$ are orthonormal (they do not have to be independent, except for Gaussian process case),

$$
\mathbb{E}\left[\xi_{i}\right]=0, \quad \mathbb{E}\left[\xi_{i} \xi_{j}\right]=\delta_{i j} .
$$


We now justify the realization generation method described above. Since Gaussian process is fully characterized by its mean and covariance function, the samples generated would satisfy such conditions,

$$
\begin{aligned}
\mathbb{E}[K(x, \omega)] & =\mathbb{E}\left[\sum_{i=1}^{\infty} \sqrt{\lambda_{i}} f_{i}(x) \xi_{i}(\omega)\right]=\sum_{i=1}^{\infty} \sqrt{\lambda_{i}} f_{i}(x) \mathbb{E}\left[\xi_{i}(\omega)\right]=0, \\
\mathbb{E}[K(x, \omega) K(y, \omega)] & =\mathbb{E}\left[\left(\sum_{i=1}^{\infty} \sqrt{\lambda_{i}} f_{i}(x) \xi_{i}(\omega)\right)\left(\sum_{j=1}^{\infty} \sqrt{\lambda_{j}} f_{j}(x) \xi_{j}(\omega)\right)\right] \\
& =\sum_{i=1}^{\infty} \sum_{j=1}^{\infty} \sqrt{\lambda_{i} \lambda_{j}} f_{i}(x) f_{j}(y) \mathbb{E}\left[\xi_{i} \xi_{j}\right] \\
& =\sum_{i=1}^{\infty} \lambda_{i} f_{i}(x) f_{i}(y) \\
& =C(x, y) .
\end{aligned}
$$

\subsubsection{Truncation Error}

In practice, the KL-expansion has to be truncated after a finite number of terms, $N_{K L}$. The mean square error is given by,

$$
\begin{aligned}
\epsilon_{N_{K L}}^{2} & =\mathbb{E}\left[\|K(x, \cdot)-\hat{K}(x, \cdot)\|_{\Omega}^{2}\right] \\
& =\mathbb{E}\left[\left\|\sum_{i=N_{K L}}^{\infty} \sqrt{\lambda_{i}} f_{i}(x) \xi_{i}(\omega)\right\|_{\Omega}^{2}\right] \\
& =\sum_{i, j>N_{K L}} \sqrt{\lambda_{i} \lambda_{j}}\left(f_{i}, f_{j}\right) \mathbb{E}\left[\xi_{i} \xi_{j}\right] \\
& =\sum_{i, j>N_{K L}} \sqrt{\lambda_{i} \lambda_{j}} \delta_{i j} \delta_{i j} \\
& =\sum_{i>N_{K L}} \lambda_{i} .
\end{aligned}
$$

A problem arises here is how to determine the number of terms $N_{K L}$ to truncate. A good idea is to investigate the decay of eigenvalues. For Matérn type covariance $(2.3)$, research $[39,4]$ show that there is an analytic asymptotic decay rate of eigenvalues,

$$
\lambda_{r} \sim \mathcal{O}\left(r^{-\frac{2 \nu+d}{d}}\right) .
$$

Schwab [111] illustrated that when $\nu=\infty$ in Eq. (2.3) the eigenvalues decays at least exponentially. Fig. 7 shows the eigenvalue decay under different covariance settings. 


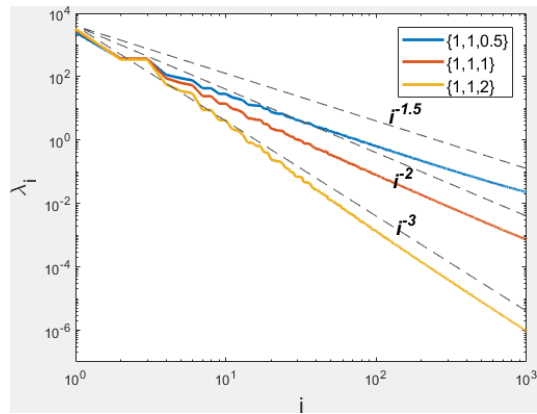

(a) Parameter $\nu$

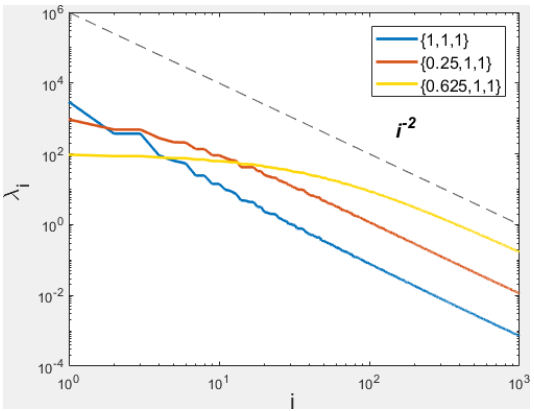

(b) Correlation length $\rho$

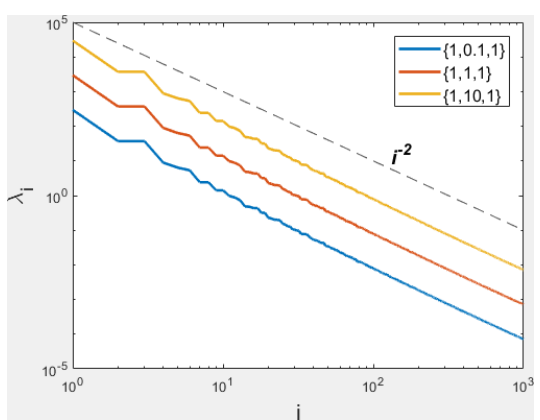

(c) Variance $\sigma^{2}$

Fig. 7 Decay of eigenvalues under different settings $\left\{\rho, \sigma^{2}, \nu\right\}$ in Matérn covariance. Dashed lines are theoretical asymptotically decay rate

\subsubsection{Numerical Determination Approach}

For specific kernel functions $C(\cdot, \cdot)$, there exists an analytic eigenvalue and eigenvector $(\lambda, f)$ solution to Eq. (2.80). When the covariance function takes the form,

$$
C(x, y)=\exp \left(-\frac{\|x-y\|_{1}}{\lambda}\right)
$$

in 1-D domain $\Omega=(0,1)$, the eigenvalues $\lambda$ and eigenfunctions $f$ are given by,

$$
\begin{aligned}
\lambda_{n}^{1 D} & =\frac{2 \lambda}{\lambda w_{n}^{2}+1}, n \in \mathbb{N}, \\
f_{n}(x)^{1 D} & =A_{n}\left(\sin \left(w_{n}\right) x+\lambda w_{n} \cos \left(w_{n} x\right)\right),
\end{aligned}
$$

where $w_{n}$ are the solutions of the following equation,

$$
\tan (w)=\frac{2 \lambda w}{\lambda^{2} w^{2}-1}
$$


and $A_{n}$ are normalizing constants such that $\left\|f_{n}\right\|_{L_{2}(\Omega)}=1$. In 2-D $\Omega=(0,1)^{2}$ domain, the eigenpairs can be expressed as,

$$
\begin{aligned}
\lambda_{n}^{2 D} & =\lambda_{i_{n}}^{1 D} \lambda_{j_{n}}^{1 D}, \\
f_{n}^{2 D}(\boldsymbol{x}) & =f_{i_{n}}^{1 D}\left(x_{1}\right) f_{j_{n}}^{1 D}\left(x_{2}\right),
\end{aligned}
$$

for some $i_{n}, j_{n} \in \mathbb{N}$.

Nevertheless in many other cases it can only be solved numerically. We now focus on a Galerkin-type approximation approach.

Let $h_{i}(x), i=1, \cdots, N_{x}$ be a set of basis functions of a Hilbert space $\mathcal{V} \subset L_{2}(\Omega)$. In such space, the eigenfunction $f_{i}(x)$ can be approximated as,

$$
f_{i}(x) \approx \sum_{k=1}^{N_{x}} d_{k}^{(i)} h_{k}(x)
$$

Substitute Eq. (2.88) into Eq. (2.80),

$$
\epsilon_{N_{k}}=\sum_{k=1}^{N_{k}} d_{k}^{(i)}\left(\int_{\Omega} C(x, y) h_{k}(y) d y-\lambda_{i} h_{k}(x)\right) .
$$

Then find coefficients $d_{k}^{(i)}$ such that the approximation error $\epsilon_{N_{k}}$ is orthogonal to the space $\mathcal{V}$, i.e.

$$
\left(\epsilon_{N_{k}}, h_{j}\right)=0 \quad \forall j \in\left\{1,2, \cdots, N_{k}\right\} .
$$

Substituting Eq. (2.89) into Eq. (2.90) gives,

$$
\sum_{k=1}^{N_{k}} d_{k}^{(i)}\left(\int_{\Omega} \int_{\Omega} C(x, y) h_{k}(y) d y \cdot h_{j}(x) d x-\int_{\Omega} \theta_{i} h_{k}(x) h_{j}(x) d x\right)=0,
$$

$\forall j \in\left\{1,2, \cdots, N_{k}\right\}$. Written in matrix form, for each $i$ we have,

$$
\left([R]_{j k}-\theta[M]_{j k}\right) \cdot d_{k}=0,
$$

where $R$ is called stiffness matrix,

$$
[R]_{j k}=\int_{\Omega} \int_{\Omega} C(x, y) h_{k}(y) d y \cdot h_{j}(x) d x,
$$

and $M$ called mass matrix,

$$
[M]_{j k}=\int_{\Omega} h_{k}(x) h_{j}(x) d x .
$$

If the basis functions $h$ are selected to be dirac functions, in particular,

$$
h_{k}(x)=\delta\left(x-x_{k}\right) .
$$

Then, the stiffness matrix $R$ becomes exactly the covariance matrix, and the mass matrix $M$ reduces to the identity matrix. This is equivalent to compute the eigenvalues and eigenvectors of the covariance matrix. 
2.6 Moving Average

The moving average (MA) method resembles the matrix decomposition method in some way. It was first applied to simulate a 1-D process by Journel [61]. Oliver [93] analyzed the method in detail for various common covariance kernels and gave simulation results in 2-D and 3-D cases.

The method first requires a decomposition of the covariance function in the following way,

$$
C(x)=f * f^{T}(x)=\int_{-\infty}^{\infty} f(s) f(x+s) d s,
$$

where $f^{T}(s)=f(-s)$

Then the random field can be given by,

$$
K(x)=\int_{-\infty}^{\infty} f(x-s) Z(s) d s=(f * Z)(x),
$$

where $Z$ is standard Gaussian random vector, i.e. $Z \sim N(0, I)$.

The above can be verified by examining the covariance structure,

$$
\mathbb{E}\left[K(x) K\left(x^{\prime}\right)\right]=\mathbb{E}\left[\int_{-\infty}^{\infty} f(x-t) Z(t) d t \int_{-\infty}^{\infty} f\left(x^{\prime}-t^{\prime}\right) Z\left(t^{\prime}\right) d t^{\prime}\right] .
$$

Notice that $\mathbb{E}\left[Z(t) Z\left(t^{\prime}\right)\right]=\delta\left(t-t^{\prime}\right)$,

$$
\begin{aligned}
\mathbb{E}\left[K(x) K\left(x^{\prime}\right)\right] & =\mathbb{E}\left[\int_{-\infty}^{\infty} f(x-t) Z(t) f\left(x^{\prime}-t\right) Z(t) d t\right] \\
& =\int_{-\infty}^{\infty} f(x-t) f\left(x^{\prime}-t\right) d t
\end{aligned}
$$

Let $s=t+x^{\prime}$,

$$
\mathbb{E}\left[K(x) K\left(x^{\prime}\right)\right]=\int_{-\infty}^{\infty} f\left(x-x^{\prime}+s\right) f(s) d s=C\left(x-x^{\prime}\right) .
$$

Oliver [93] proved that the method can be easily extended to 2- or 3- D and provided the decomposition for some given covariance functions.

It should be noted that the Fourier transform of the convolution is equal to the product of Fourier transform, i.e.,

$$
\mathcal{F}(g * h)=\mathcal{F}(g) \cdot \mathcal{F}(h) .
$$

The random field $K(x)$ is then given by,

$$
\begin{aligned}
K(x) & =\mathcal{F}^{-1}\{\mathcal{F}[(f * Z)(x)]\} \\
& =\mathcal{F}^{-1}\{\mathcal{F}(f) \cdot \mathcal{F}(Z)\} .
\end{aligned}
$$



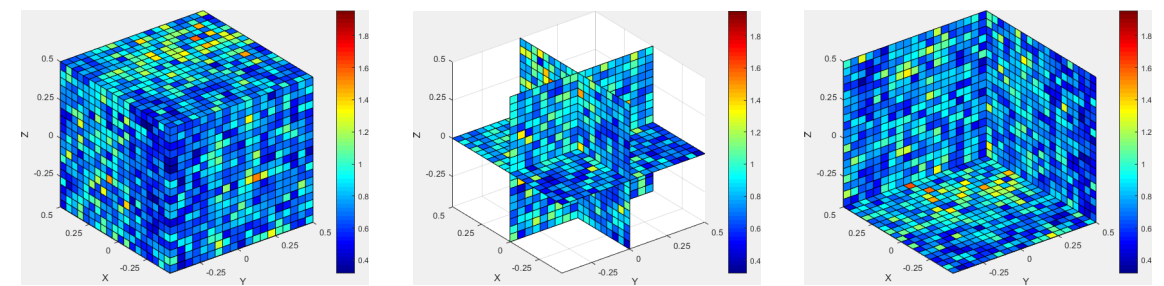

Fig. 8 An example of isotropic log-normal Gaussian random field generated by FFT-MA method, $\log K \sim N(0,1), C(r)=\exp \left(-r^{2}\right)$

Le Ravalec [69] exploited the acceleration of FFT. In the 1-D case where there are $N$ points and the sampling rate is $d x$, the frequency rate is correspondingly $d f=\frac{1}{N d x}$ and the frequency range is $\left[-\frac{1}{2 d x}, \frac{1}{2 d x}\right]$. Then the relation between covariance function $C$ and spectral density $S$ is given by,

$$
S\left(j_{1}\right)=d x \sum_{k=1}^{N} C(k d x) \exp \left(-2 i \pi \frac{k j_{1}}{N}\right), \quad j_{n}=\frac{2 n-N}{2 N d x}, n=1, \cdots, N .
$$

The Fourier transform of Eq. (2.96) is given by,

$$
S(j)=\frac{1}{d x} F(j) F^{*}(j) .
$$

Then the Fourier transform of $f$, denoted as $F$, can be obtained by,

$$
F(j)=\sqrt{d x S(j)} .
$$

The random field $K$ is finally obtained as follows,

$$
K=\mathcal{F}^{-1}(F \cdot \mathcal{F}(Z)) .
$$

It may not always happen that the covariance kernel $C$ has an analytic decomposition $f$, but when considering the Fourier transformation, a square root $F$ can be easily obtained for many covariance functions. With the help of FFT, the generation process is fast. Fig. 8 is an example of FFT-MA generated 3-D random field.

\subsection{Sequential Simulation}

Sequential simulation $[99,60,38]$ in a way can be viewed as a conditional random field generation method. The probability density function of multidimensional random variable can be written as,

$$
\begin{aligned}
p\left(x_{1}, x_{2}, \cdots, x_{N}\right) & =p\left(x_{2}, \cdots, x_{N} \mid x_{1}\right) \cdot p\left(x_{1}\right) \\
& =p\left(x_{3}, \cdots, x_{N} \mid x_{2}, x_{1}\right) \cdot p\left(x_{2} \mid x_{1}\right) \cdot p\left(x_{1}\right) .
\end{aligned}
$$


The above equation can be written recursively, which indicates that we can first generate $x_{1}$, then generate $x_{2}$ conditional to $x_{1}$ and so on. This reduces the multivariate problem to a sequence of uni-dimensional problem.

Suppose $M$ data points have been obtained either from prior information or from simulation to generate $Z_{j}$. The conditional distribution of $Z_{j} \mid\{M\}$ is still Gaussian, the mean and variance can be determined by kriging,

$$
\begin{aligned}
& \mathbb{E}\left[Z_{j} \mid\{M\}\right]=\mu+\sum_{i=1}^{M} \lambda_{i}\left(z_{i}-\mu\right), \\
& \mathbb{V}\left[Z_{j} \mid\{M\}\right]=\sigma^{2}-\sum_{i=1}^{M} \lambda_{i} C\left(x_{j}-x_{i}\right),
\end{aligned}
$$

where the coefficients $\lambda_{1}, \cdots, \lambda_{M}$ are the solutions to the following kriging equation,

$$
\sum_{i=1}^{M} \lambda_{i} C\left(x_{i}-x_{i^{\prime}}\right)=C\left(x_{j}-x_{i^{\prime}}\right), \quad i^{\prime}=1, \cdots, M .
$$

Substitute Eq. (2.109) into Eq. (2.108), the conditional mean and variance (posterior) can be obtained,

$$
\begin{aligned}
& \mathbb{E}\left[Z_{j} \mid\{M\}\right]=\mu_{j}+C_{j M} C_{M M}^{-1}\left(Z_{m}-\mu_{M}\right), \\
& \mathbb{V}\left[Z_{j} \mid\{M\}\right]=C_{j} j-C_{j M} C_{M M}^{-1} C_{M j},
\end{aligned}
$$

The major computational cost comes from $C_{M M}^{-1}$, which is $\mathcal{O}\left(M^{3}\right)$. Suppose the prior information consists $M$ points and there are $N$ points to simulate, the total cost will be $\sum_{i=1}^{N} \mathcal{O}\left((M+i)^{3}\right)=\mathcal{O}\left((M+N)^{4}\right)$. As more and more samples are simulated and joined in the prior information, the computation cost of $C_{M M}^{-1}$ becomes prohibitive. Then in practice, in the computation of posterior, only a local prior information $M_{l o c}$, which comprises of neighboring points, is used,

$$
\begin{aligned}
\mathbb{E}\left[Z_{j} \mid M\right] & \approx \mathbb{E}\left[Z_{j} \mid M_{l o c}\right], \\
\mathbb{V}\left[Z_{j} \mid M\right] & \approx \mathbb{V}\left[Z_{j} \mid M_{l o c}\right],
\end{aligned}
$$

where $Z_{j}$ denotes the vector consisting the points in $j_{t h}$ group. $M_{l o c}$ is then the neighbouring points of the whole group. By limiting the neighbourhood size $\nu_{\max }$, the computational cost is reduced to $\mathcal{O}\left(\left(M \nu_{\max }\right)^{3}\right)$.

Dimitrakopoulos [22] proposed to simulate a group of points simultaneously. Generally, $N$ total data points can be divided into $k$ groups. For simplicity, let each group have same size $\nu, \nu=N / k$, then the computational cost reduces to $\mathcal{O}\left(\frac{N}{\nu}\left(\nu_{\max }^{3}+\nu^{3}\right)\right.$. The cost reaches minimum when $\nu=\nu_{\max } / \sqrt[3]{2}$.

Sequential simulation does not depend on the covariance kernel, points locations, and has low memory requirement. In real-world applications, simulation of tens of thousands nodes is not unusual. Then sequential simulation method is highly suitable and widely adopted in random field simulation software $[121,108,98,73,133]$. 
2.8 Local Average Subdivision

\subsubsection{Local Average}

Local Average Subdivision (LAS) method was first proposed by Fenton[34]. Many subsequent works $[51,31,120,92,24]$ contribute to the development of this method. Before introducing the LAS method, the ingredients for later use are provided first.

The local average $X_{T}(t)$ of a Gaussian random field $K$ within interval $[t-T / 2, t+T / 2]$ is given by,

$$
X_{T}(t)=\frac{1}{T} \int_{t-T / 2}^{t+T / 2} K(u) d u .
$$

The integral is known as the local integral process $I_{T}(t)$, then the local average is only different to the local integral up to a constant factor,

$$
X_{T}(t)=\frac{1}{T} I_{T}(t) .
$$

Fig. 9 presents an example of the original process $X(t)$, the local average process $X_{T}(t)$ and the local integration process $I_{T}(t)$.

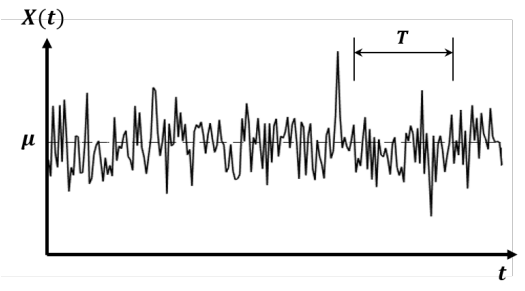

(a) Random Process $X(t)$

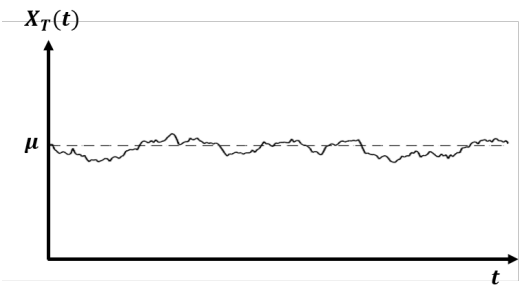

(b) Local Averaging $X_{T}(t)$

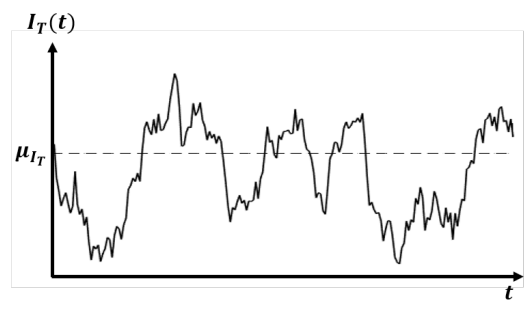

(c) Local Integration $I_{T}(t)$

Fig. 9 An example of random process $X(t)$ and the corresponding local averaging process $X_{T}(t)$ and integration process $I_{T}(t)$, Vanmarcke (1983) [128]

To find the statistical moments of the random variable $X_{T}(t)$, since $K(\cdot)$ is Gaussian, $X_{T}(t)$ and $I_{T}(t)$ are also Gaussian random variables, the expectation and variance are, 


$$
\begin{aligned}
\mathbb{E}\left[X_{T}(t)\right]=\mathbb{E}\left[\frac{1}{T} \int_{t-T / 2}^{t+T / 2} K(u) d u\right]=\mathbb{E}[K(u)]=\mu, \\
\mathbb{V}\left[X_{T}(t)\right]=\mathbb{E}\left[\left(X_{T}(t)-\mu\right)^{2}\right] \\
=\frac{1}{T^{2}} \mathbb{E}\left[\int_{t-\frac{T}{2}}^{t+\frac{T}{2}} \int_{t-\frac{T}{2}}^{t+\frac{T}{2}}(K(u)-\mu)\left(K\left(u^{\prime}\right)-\mu\right) d u d u^{\prime}\right] \\
=\frac{\sigma^{2}}{T^{2}} \int_{t-\frac{T}{2}}^{t+\frac{T}{2}} \int_{t-\frac{T}{2}}^{t+\frac{T}{2}} \mathbb{E}\left[\frac{(K(u)-\mu)\left(K\left(u^{\prime}\right)-\mu\right)}{\left.\sqrt{\mathbb{V}[K(u)}] \sqrt{\mathbb{V}\left[K\left(u^{\prime}\right)\right]}\right] d u d u^{\prime}}\right. \\
=\frac{\sigma^{2}}{T^{2}} \int_{t-\frac{T}{2}}^{t+\frac{T}{2}} \int_{t-\frac{T}{2}}^{t+\frac{T}{2}} \rho\left(K(u), K\left(u^{\prime}\right)\right) d u d u^{\prime},
\end{aligned}
$$

where $\rho(\cdot, \cdot)$ is Pearson's correlation coefficient. The above equation can be further simplified by the introduction of variance function $\gamma$,

$$
\begin{aligned}
\gamma(T) & =\frac{1}{T^{2}} \int_{t-\frac{T}{2}}^{t+\frac{T}{2}} \int_{t-\frac{T}{2}}^{t+\frac{T}{2}} \rho\left(K(u), K\left(u^{\prime}\right) d u d u^{\prime}\right. \\
& =\frac{2}{T} \int_{0}^{T} \rho(\tau) d \tau-\frac{2}{T^{2}} \int_{0}^{T} \tau \rho(\tau) d \tau .
\end{aligned}
$$

Then,

$$
\mathbb{V}\left[X_{T}(t)\right]=\gamma(T) \sigma^{2} .
$$

Accordingly the variance of local integration process is,

$$
\mathbb{V}\left[I_{T}(t)\right]=T^{2} \gamma(T) \sigma^{2}=\Delta(T) \sigma^{2} .
$$

Since $0 \leq \gamma(T) \leq 1$, then the variance function $\gamma$ measures how much the variance is reduced in the local average process $X_{T}(t)$.

Now consider the covariance function between two discrete local integration $I_{T}$ and $I_{T}^{\prime}$, as shown in Fig. 10,

From the definition of local integral process, the following equations can be easily obtained,

$$
\begin{aligned}
I_{T} & =I_{T_{2}}-I_{T_{3}}=I_{T_{1}}-I_{T_{0}}, \\
I_{T^{\prime}} & =I_{T_{2}}-I_{T_{1}}=I_{T_{3}}-I_{T_{0}} .
\end{aligned}
$$

Then we can derive that,

$$
2 T T^{\prime}=\sum_{k=0}^{3}(-1)^{k} I_{T_{k}}^{2}
$$




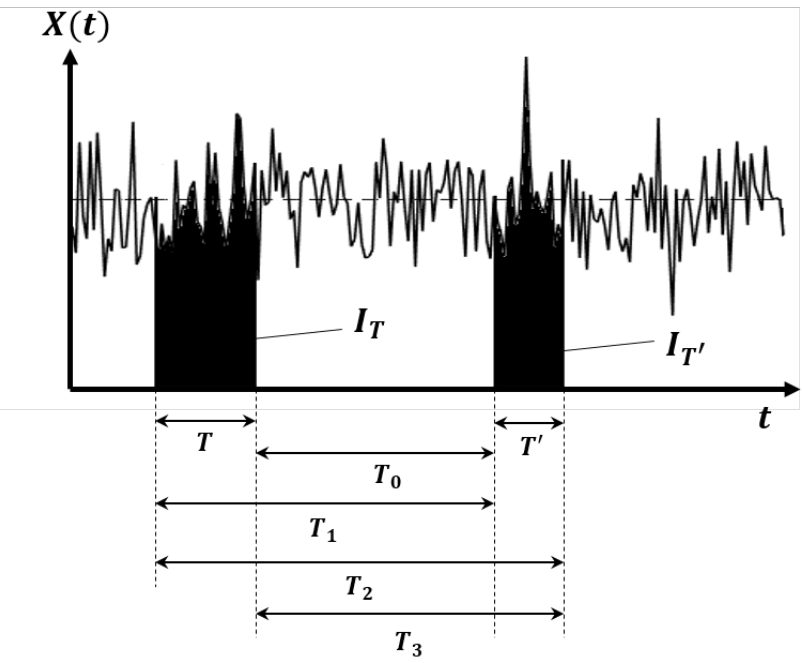

Fig. 10 A schematic local integration, Nuttall (2011) [92]

For simplicity here the random process $K$ is assumed to have mean $\mu$ equals zero, then the covariance between $I_{T}$ and $I_{T}^{\prime}$ is given by,

$$
\operatorname{Cov}\left[I_{T}, I_{T^{\prime}}\right]=\mathbb{E}\left[I_{T} I_{T^{\prime}}\right]=\frac{\sigma^{2}}{2} \sum_{k=0}^{3}(-1)^{k} \Delta\left(T_{k}\right)
$$

If the blocks are equi-spaced, and let $T_{1}=T_{3}=\tau, T_{0}=\tau-T$ and $T_{2}=T+\tau$, the covariance is,

$$
\operatorname{Cov}\left[I_{T}, I_{T^{\prime}}\right]=\frac{\sigma^{2}}{2}\left[(T-\tau)^{2} \gamma(T-\tau)-2 \tau^{2} \gamma(\tau)+(T+\tau) \gamma(T+\tau)\right] .
$$

\subsubsection{LAS Implementation}

This part explains how LAS method works. As shown in Fig. 11, LAS first assigns an averaged value to the whole domain, then the domain is divided into subdomains and averaged values are assigned to these subdomains. The process is done recursively until a final resolution is reached, where the random field is represented by these local averages.

In the 1-D case, a domain is always divided into two subdomains, thus on stage $i$ there are totally $2^{i}$ subdomains. Number the cells so that the son cells of father cell $Z_{j}^{i}$ are $Z_{2 j-1}^{i+1}$ and $Z_{2 j}^{i+1}$. The value of the $2 j_{t h}$ cell on stage $i+1$ is given by its mean value $M_{2 j}^{i+1}$ plus a magnified white noise term $c^{i+1} U_{2 j}^{i+1}$, where $U \sim N(0,1)$ and $c^{i+1}$ is a constant only depends on the stage $i+1$,

$$
Z_{2 j}^{i+1}=M_{2 j}^{i+1}+c^{i+1} U_{2 j}^{i+1} .
$$




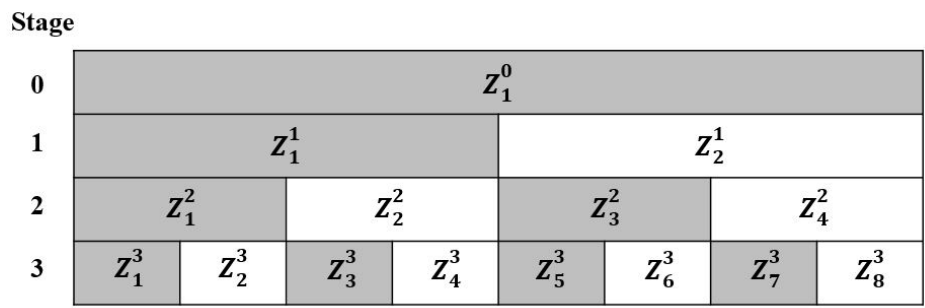

Fig. 11 LAS method in the 1-D case, Nuttall (2011) [92]

The mean value $M_{2 j}^{i+1}$ depends on the values on the previous level, it is assumed that the close neighbourhood of three elements that matter,

$$
M_{2 j}^{i+1}=a_{-1}^{i} Z_{j-1}^{i}+a_{0}^{i} Z_{j}^{i}+a_{1}^{i} Z_{j+1}^{i} .
$$

Substitute Eq. (2.124) into Eq. (2.123) gives the expression for $Z_{2 j}^{i+1}$,

$$
Z_{2 j}^{i+1}=a_{-1}^{i} Z_{j-1}^{i}+a_{0}^{i} Z_{j}^{i}+a_{1}^{i} Z_{j+1}^{i}+c^{i+1} U_{2 j}^{i+1} .
$$

Multiply $Z_{m}^{i}$, which is the value in $m_{t h}$ cell in the previous stage, and take the expectation,

$$
\mathbb{E}\left[Z_{2 j}^{i+1} Z_{m}^{i}\right]=\sum_{j-1}^{j+1} a_{k-j}^{i} \mathbb{E}\left[Z_{k}^{i} Z_{m}^{i}\right]+c^{i+1} \mathbb{E}\left[Z_{m}^{i} U_{m}^{i+1}\right] .
$$

Since the white noise term $U_{m}^{i+1}$ has zero mean and is independent of the term $Z_{m}^{i}$, the last term in Eq. (2.126) is zero. Now let $m=j-1, j, j+1$,

$$
\left[\begin{array}{c}
\mathbb{E}\left[Z_{2 j}^{i+1} Z_{j-1}^{i}\right] \\
\mathbb{E}\left[Z_{2 j}^{i+1} Z_{j}^{i}\right] \\
\mathbb{E}\left[Z_{2 j}^{i+1} Z_{j+1}^{i}\right]
\end{array}\right]=\left[\begin{array}{ccc}
\mathbb{E}\left[Z_{j-1}^{i} Z_{j-1}^{i}\right] & \mathbb{E}\left[Z_{j}^{i} Z_{j-1}^{i}\right] & \mathbb{E}\left[Z_{j+1}^{i} Z_{j-1}^{i}\right] \\
\mathbb{E}\left[Z_{j-1}^{i} Z_{j}^{i}\right] & \mathbb{E}\left[Z_{j}^{i} Z_{j}^{i}\right] & \mathbb{E}\left[Z_{j+1}^{i} Z_{j}^{i}\right] \\
\mathbb{E}\left[Z_{j-1}^{i} Z_{j+1}^{i}\right] & \mathbb{E}\left[Z_{j}^{i} Z_{j+1}^{i}\right] & \mathbb{E}\left[Z_{j+1}^{i} Z_{j+1}^{i}\right]
\end{array}\right] \cdot\left[\begin{array}{c}
a_{-1}^{i} \\
a_{0}^{i} \\
a_{1}^{i}
\end{array}\right]
$$

The matrix on the right hand side of Eq. (2.127) is symmetric Toeplitz and can be evaluated by Eq. (2.122), while the vector on the left hand side is the covariance between stages and can be further calculated by exploiting the mean preserving property,

$$
\left[\begin{array}{c}
\mathbb{E}\left[Z_{2 j}^{i+1} Z_{j-1}^{i}\right] \\
\mathbb{E}\left[Z_{2 j}^{i+1} Z_{j}^{i}\right] \\
\mathbb{E}\left[Z_{2 j}^{i+1} Z_{j+1}^{i}\right]
\end{array}\right]=\frac{1}{2}\left[\begin{array}{c}
\mathbb{E}\left[Z_{2 j}^{i+1} Z_{2 j-3}^{i+1}\right]+\mathbb{E}\left[Z_{2 j}^{i+1} Z_{2 j-2}^{i+1}\right] \\
\mathbb{E}\left[Z_{2 j}^{i+1} Z_{2 j-1}^{i+1}\right]+\mathbb{E}\left[Z_{2 j}^{i+1} Z_{2 j}^{i+1}\right] \\
\mathbb{E}\left[Z_{2 j}^{i+1} Z_{2 j+1}^{i+1}\right]+\mathbb{E}\left[Z_{2 j}^{i+1} Z_{2 j+2}^{i+1}\right]
\end{array}\right]
$$

the right hand side of Eq. (2.128) can also be evaluted by Eq. (2.122). Thus the constants $a_{-1}^{i}, a_{0}^{i}, a_{1}^{i}$ can be determined, then $c^{i+1}$ needs to be determined. Square Eq. (2.123) and take expectation,

$$
\mathbb{E}\left[\left(Z_{2 j}^{i+1}\right)^{2}\right]=\mathbb{E}\left[\left(M_{2 j}^{i+1}\right)^{2}\right]+\left(c^{i+1}\right)^{2},
$$


where the expectation on the right hand side is given by,

$$
\begin{aligned}
\mathbb{E}\left[\left(M_{2 j}^{i+1}\right)^{2}\right] & =\mathbb{E}\left[\left(Z_{2 j}^{i+1}-c^{i+1} U_{j}^{i+1}\right)\left(a_{-1}^{i} Z_{j-1}^{i}+a_{0}^{i} Z_{j}^{i}+a_{1}^{i} Z_{j+1}^{i}\right)\right] \\
& =\sum_{k=j-1}^{j+1} a_{k-j}^{i} \mathbb{E}\left[Z_{2 j}^{i+1} Z_{k}^{i}\right],
\end{aligned}
$$

with the coefficients $a$ determined, $c$ can be calculated.

For cases where the number of parent cells is less than 3, for instance the two cells in stage 1, whose parent cell is the whole domain, Fenton and Vanmarcke [34] suggested that the cells outside domain can be neglected, i.e. in Eq. (2.124) the $Z^{i}$ value is taken to be zero if the corresponding cell is outside the domain.

The idea can be generalized to multi-dimensional case. Only the Eq. (2.123) and (2.124) are different,

$$
\begin{aligned}
Z_{2 j, 2 k}^{i+1} & =c_{11} U_{1}^{i+1}+\sum_{l=1}^{n_{x y}} a_{l_{1}}^{i} Z_{m(l), n(l)}^{i}, \\
Z_{2 j, 2 k-1}^{i+1} & =c_{21} U_{1}^{i+1}+c_{22} U_{2}^{i+1}+\sum_{l=1}^{n_{x y}} a_{l_{2}}^{i} Z_{m(l), n(l)}^{i}, \\
Z_{2 j-1,2 k}^{i+1} & =c_{21} U_{1}^{i+1}+c_{22} U_{2}^{i+1}+c_{33} U_{3}^{i+1}+\sum_{l=1}^{n_{x y}} a_{l_{3}}^{i} Z_{m(l), n(l)}^{i}, \\
Z_{2 j-1,2 k-1}^{i+1} & =4 Z_{j, k}^{i}-Z_{2 j, 2 k}^{i+1}-Z_{2 j, 2 k-1}^{i+1}-Z_{2 j-1,2 k}^{i+1} .
\end{aligned}
$$

Also notice that in the 2-D case a neighbourhood of $3 \times 3$ cells is considered. Reader can check $[34,31,92]$ for more details.

\subsection{Summary}

In this Section seven popular random field generation methods were introduced. For the sake of choosing these methods according to specific requirements, in the following these methods are summarized and compared in terms of its algorithm complexity, computational cost and memory requirement, covariance kernel requirement and grid requirement.

(1) Algorithm complexity. The matrix decomposition, KL expansion and MA methods are straightforward to implement. The spectral method involves discrete Fourier transform or FFT, in which we need to sample $d W$ first and in FFT case we need to take special care to the grid and frequency increments. The sequential simulation requires the computation of expected mean and variance before each conditional simulation. The TBM requires a 1-D representation of the original random field, after that the line process is generated with spectral method. The LAS method involves recursively average 
subdivision and in each step a linear algebraic equation system needs to be solved.

(2) Computational cost and memory requirements. The TBM costs as much as generating a series of line processes. The sequential method generates a random field of far less dimension at each step. The LAS involves the generation of individual Gaussian random variables, whose mean and variance are computed through a local algebraic equation system. The spectral method involves the generation of Fourier increments $d W$ and multiplication with Fourier transform matrix. The MA involves convolution of kernel function and standard Gaussian random variables. The process can be accelerated by the FFT. The KL expansion uses a low-rank approximation to the covariance function, then multiplies eigenvalues, eigenfunctions and standard Gaussian random vector. The matrix decomposition method is the most expensive method, the cost of which includes Cholesky decomposition and matrix-vector multiplication.

(3) Applicability : Covariance function, grid type and accuracy. The TBM works for the specific covariance function, which has corresponding 1-D covariance or spectral representation. It can simulate points of arbitrary position and the simulation is an approximation to the exact random field. The spectral method works for covariance functions whose power spectral density can be obtained. The discrete Fourier transform is comparatively slow and will diminish the method's advantage, thus here we discuss the FFT method. In the FFT method, the grid needs to be rectangular. Since the FFT truncates the integration in finite bandwidth, the simulation is an approximation. The matrix decomposition works for all covariance functions, it can generate the random field on all types of grids and the simulation is exact. The MA method works for specific covariance function, which has decomposition in convolution sense. The convolution can be accelerated by the FFT, but in the FFT-MA case, the discrete decomposition can be obtained. The FFT-MA method is an approximation and works on rectangular grid. In discrete case, the KL expansion works for all kinds of covariance functions, it can generate random fields on arbitrary position. Since it is a low-rank representation, the simulation is an approximation. The sequential simulation essentially generates a part of random field recursively by using any other methods, thus the applicability depends on the exact method chosen. The LAS works for any covariance kernel type, it generates random fields on rectangular grid and the simulation is an approximation.

To sum up, we list the comparisons in Table 2.

For readers' convenience, we provide four representative example codes of Gaussian random field generation methods (TBA, matrix decomposition, KL-expansion and MA methods respectively) in Appendix A. 
Table 2 Comparison of Gaussian random field generation methods

\begin{tabular}{lllll}
\hline & $\begin{array}{l}\text { Algorithm } \\
\text { Complexity }\end{array}$ & Covariance & Grid & Accuracy \\
& $\mathcal{O}(N \log N)$ & Specific & All & Inexact \\
TBM & $\mathcal{O}(N \log N)$ & Specific & Rectangular & Inexact \\
Matrix Decomposition & $\mathcal{O}\left(N^{3}\right)$ & All & All & Exact \\
MA & $\mathcal{O}(N \log N)$ & Specific & All & Inexact \\
KL-expansion & $\mathcal{O}\left(N^{3}\right)$ & All & All & Inexact \\
Sequential simulation & $\mathcal{O}(N)$ & All & All & Inexact \\
LAS & $\mathcal{O}(N)$ & All & Rectangular & Inexact \\
\hline
\end{tabular}

\section{Application of the Gaussian random field}

In the last few years, the advance in random field theory is accompanied by its application in a great many scientific and engineering problems. In this Section, we will briefly review general applications of the random field. It should be noted that since the establishment of random field theory, it has been applied to a wide range of problems for more than 40 years, thus in this Section, we only review and summarize some representative application areas of the Gaussian random field in recent two decades, e.g., subsurface flow and transport in porous media, slope stability analysis, settlement and consolidation, structural materials in civil engineering, etc. For the applications of Gaussian random field before the year 2000, readers of interest can refer to the review by Abrahamsen [1] or other related textbooks for more details.

\subsection{Subsurface flow and transport in porous media}

Numerical simulations of flow and transport in underground porous media (e.g., oil/gas flow in reservoir, underground containment transport) are always subject to uncertainties mainly stemming from the inherent spatial heterogeneity of media properties caused by complex geological processes. It has been widely recognized that in natural porous media most parameters, e.g. permeability, porosity, etc., exhibit an uneven spatial distribution. For example, the hydraulic conductivity would span several orders of magnitude in one formation. Therefore, a reasonable representation of uncertainties in underground porous media is of great significance in subsurface simulations. A possible way to deal with uncertainties is to treat porous media properties as random fields.

In real-world subsurface simulations, the hydraulic conductivity is a crucial control on fluid motions in porous media. Teatini et al. (2010) [125] analyzed the effects that spatially heterogeneous hydraulic conductivity distributions in the injected formations may have on the uniformity of the induced Venice land uplift. In their study, the heterogeneous hydraulic conductivity was specified as a spatially-correlated second-order random field characterized by a Gaussian distribution with an exponential covariance function in log-transformed space (see Fig.12), which was generated using the direct Fourier transform method. 
Schlter and Vogel (2011) [109] investigated the potential of various morphological descriptors to predict flow and transport in heterogeneous porous media. They adopted the multivariate Gaussian random fields and their transformations to denote heterogeneous hydraulic properties regarding the water retention characteristic and hydraulic conductivity (see Fig.13). These generated random fields were used as reference images with well-defined structural attributes.

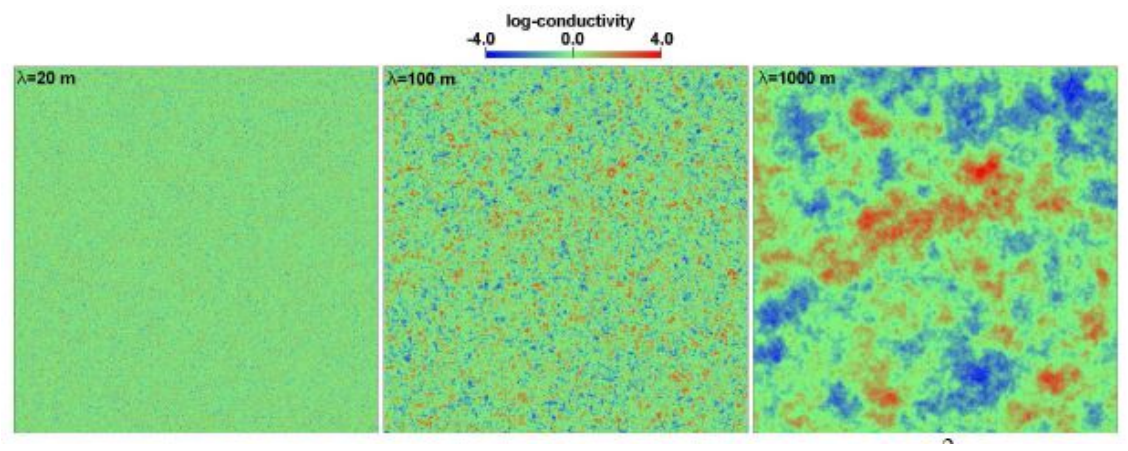

Fig. 12 Examples of hydraulic conductivity field generations obtained with variance of 1.0 and isotropic correlation length values of 20, 100, and 1,000 m, Teatini et al. (2010)[125]

Riahi et al. (2012) [103] studied effects of variations in the permeability and its spatial correlation on the hydro-mechanical behavior of Large Open Pit problems in a 2-D framework by using a probabilistic analysis approach, the random values of the permeability were assumed to be spatially correlated in underlying Gaussian fields. Boschan and Ntinger (2012) [11] investigated the statistical properties of effective hydraulic conductivity distributions and their dependence on the coarse-scale in 3-D heterogeneous media. They employed the FFT-MA method to generate random fields following a lognormal law. Beaudoin et al. (2013) [6] analyzed the convergence of macro spreading using flow and transport model for an inert solute in 3-D porous media, where the conductivity was modeled using a random field following a second-order stationary Gaussian distribution. Benson et al. (2013) [8] studied the fractional calculus in hydrologic modeling from the numerical perspective. In their study the hydraulic conductivity was assumed as an unconditional Gaussian random field. Zeng et al. (2013) [134] built a synthetic groundwater model and constructed three alternative conceptual models, in which the standard Gaussian random fields of hydraulic conductivity for the modeling aquifers were generated by direct Fourier transform method. Alkhatib (2014) [3] applied the multilevel Monte Carlo method to quantify spatial uncertainties for chemical EOR processes (e.g., polymer and surfactant-polymer flooding processes), the permeability was assumed as the lognormal permeability field (see Fig.14) and generated by means of MA method. 


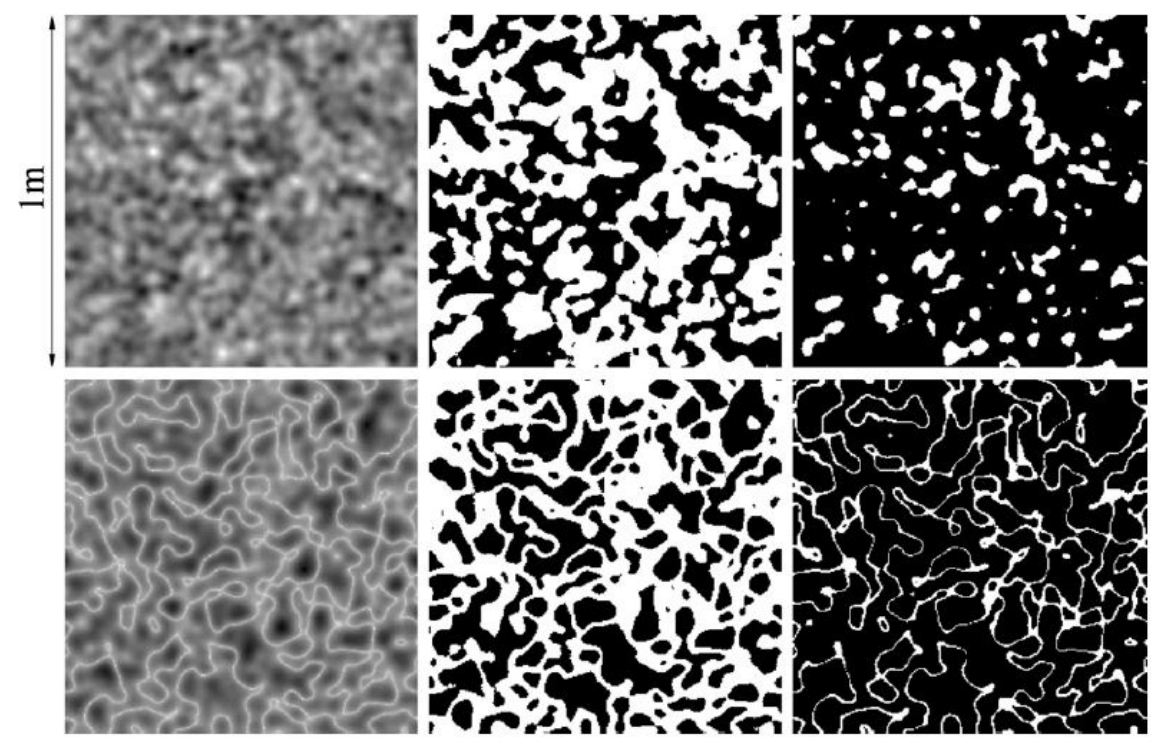

Fig. 13 Left: multivariate Gaussian random field (top) and its transformation with connected bands of high values (bottom); Middle: thresholded at percolation higher than the threshold; Right: thresholded at percolation equal (lower than) the threshold. Schlter et al. (2011) [109]

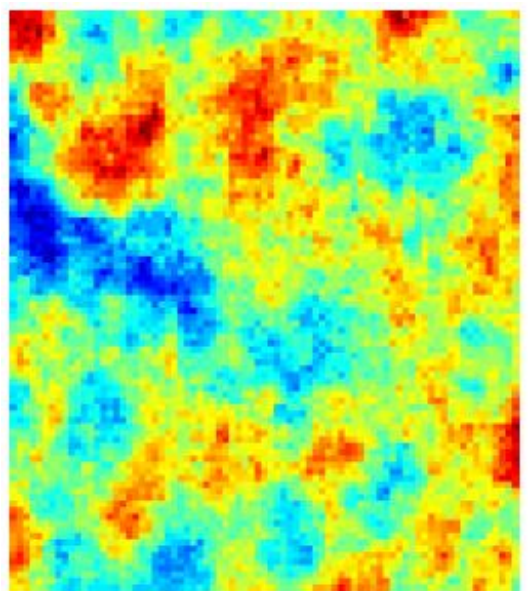

Fig. 142 -D reservoir models generated by moving average method on $120 \times 120$ grids, Alkhatib (2014) [3]

Tian et al. (2015) [126] presented a novel method to study influences of porosity and permeability heterogeneities on the alteration of minerals, the associated evolution of caprock sealing, and containment of supercritical $\mathrm{CO}_{2}$ in the caprock. The permeability and porosity fields were created following a lognormal distribution in 2-D caprock (see Fig.15), and the correlation matrix 
was established and decomposed by Cholesky factorization. Drakos and Pande (2015) [23] adopted the Polynomial Chaos approach to perform stochastic finite element analyses for transport phenomena in geomechanics, the diffusion and convection coefficients and the source/sink were considered as secondorder random fields following the lognormal distribution. Guo and Brusseau (2017) [45] investigated the influence of well-field heterogeneities of permeability and hydraulics on mass-removal efficiency for systems consisting of large groundwater contaminant plumes layer. The permeability was modeled as a stationary, lognormally distributed field and generated using the FFT method.

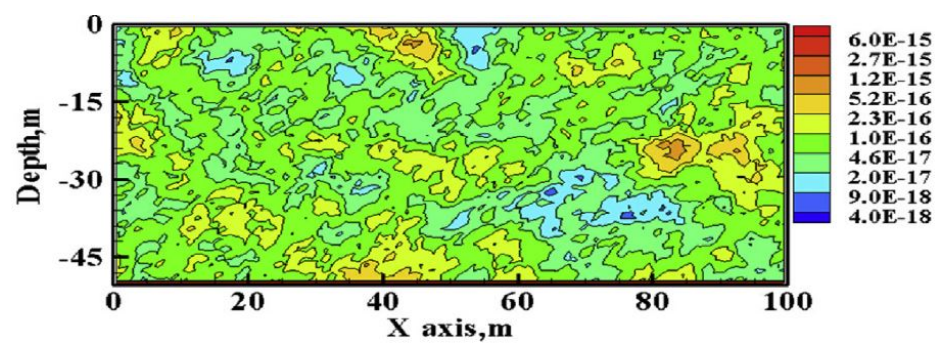

(a) Spatial distribution of the initial permeability

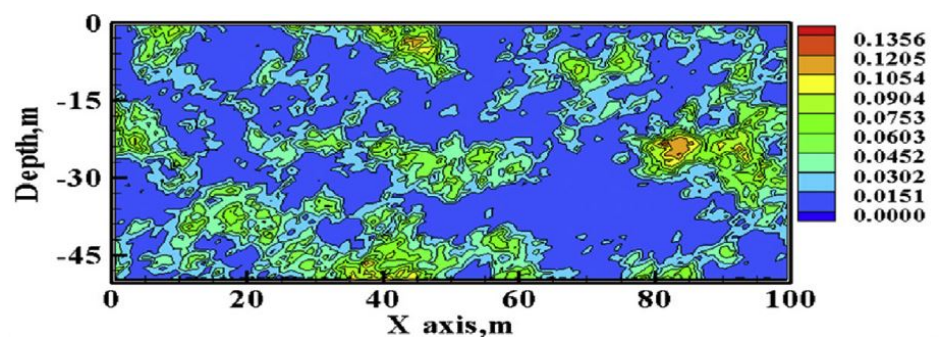

(b) Spatial distribution of the initial porosity

Fig. 15 Permeability and porosity random fields, Tian et al. (2015) [126]

\subsection{Slope stability analysis}

Slope stability analysis is a significant branch of geotechnical engineering which mainly focuses on the resistance evaluation of a natural or human-made slope to the failure by sliding along any slip surface, and the safety margin assessment of a slope against the sliding. Generally the uncertainties are unavoidable in slope engineering, among which the inherent spatial variability of geotechnical properties is deemed to be one of the major uncertainty sources. For instance, the largest uncertainty always originates from mechanical properties of the soil material, sometimes even in homogeneous layer the soil properties show inherent spatial variability. In commonly-used methods for slope stability analysis, e.g. random finite element method (RFEM), the 
random field is usually employed to describe the inherent spatial variability of soil properties.

The last two decades have witnessed the progress in methodologies for the probabilistic slope stability analysis accounting for the random characteristic of soil properties. Griffiths et al. (2004) [41], (2009) [44], (2011) [43] investigated the failure probability of slopes by RFEM considering the influence of spatial variability of soil properties. In their study, the soil shear strength, porosity and void size, etc., were considered as Gaussian distributed fields generated by LAS method. Jiang et al. (2014) [58] proposed a method to efficiently evaluate the failure probability of slope stability in spatially variable soils based on the Monte Carlo Simulation (MCS). Random field theory was applied to explicitly describe relevant soil properties, such as the undrained shear strength, cohesion, and friction angle, in horizontal and vertical directions with 2-D lognormal stationary random fields. Chenari and Alaie (2015) [55] investigated the effect of undrained shear strength heterogeneity on the performance of clay slope using MCS-based approach. In their work the undrained shear strength was treated as a lognormally distributed random variable throughout the whole domain with regard to the constant and depth-varying means. Chen et al. (2015) [14] conducted the probabilistic analysis of embankment slope stability in frozen ground regions using RFEM with consideration of inherent uncertainties of soil properties, the KL expansion was applied to attain the Gaussian random fields for hydraulic and thermal conductions. Griffiths et al. (2015) [42] performed the probabilistic slope stability analysis on an undrained slope and the non-stationary Gaussian random field was generated with linearly increasing mean undrained strength and a constant coefficient of variation. Huang and Griffiths (2015) [52] studied the determination of appropriate finite element size for modeling the strength of undrained random soils. The strength of soil materials was assumed as Gaussian random fields using local averaging and KL expansion methods for random field generation. Jha (2015) [57] investigated influences of the spatial variability of soil properties on slope reliability using three methods: RFEM, first order second moment (FOSM) method and its modification. The spatially varying nature of soil properties, such as the undrained shear strength, were described by stationary lognormal random fields (see Fig.16) using LAS method.

Johari et al. (2015) [59] proposed a RFEM-based approach to assess the reliability of static liquefaction of saturated loose sand under monotonic loading. Soil parameters, e.g., saturated unit weight, peak friction angle, and initial plastic shear modulus, were modeled by a 2-D Gaussian random field generated using Cholesky decomposition. Liu et al. (2015) [76] briefly studied the effect of spatial variability in the foundation hydraulic conductivity on the local velocity variation of seepages through and beneath an embankment for piping investigation. The hydraulic conductivity was lognormally distributed using LAS method and RFEM was applied to incorporate the uncertainty existing in the hydraulic conductivity. Zhu et al. (2015) [141] investigated the failure mechanism of slopes in random undrained soils with various slope angles by means of RFEM. The undrained shear strength was considered as an isotropic, 

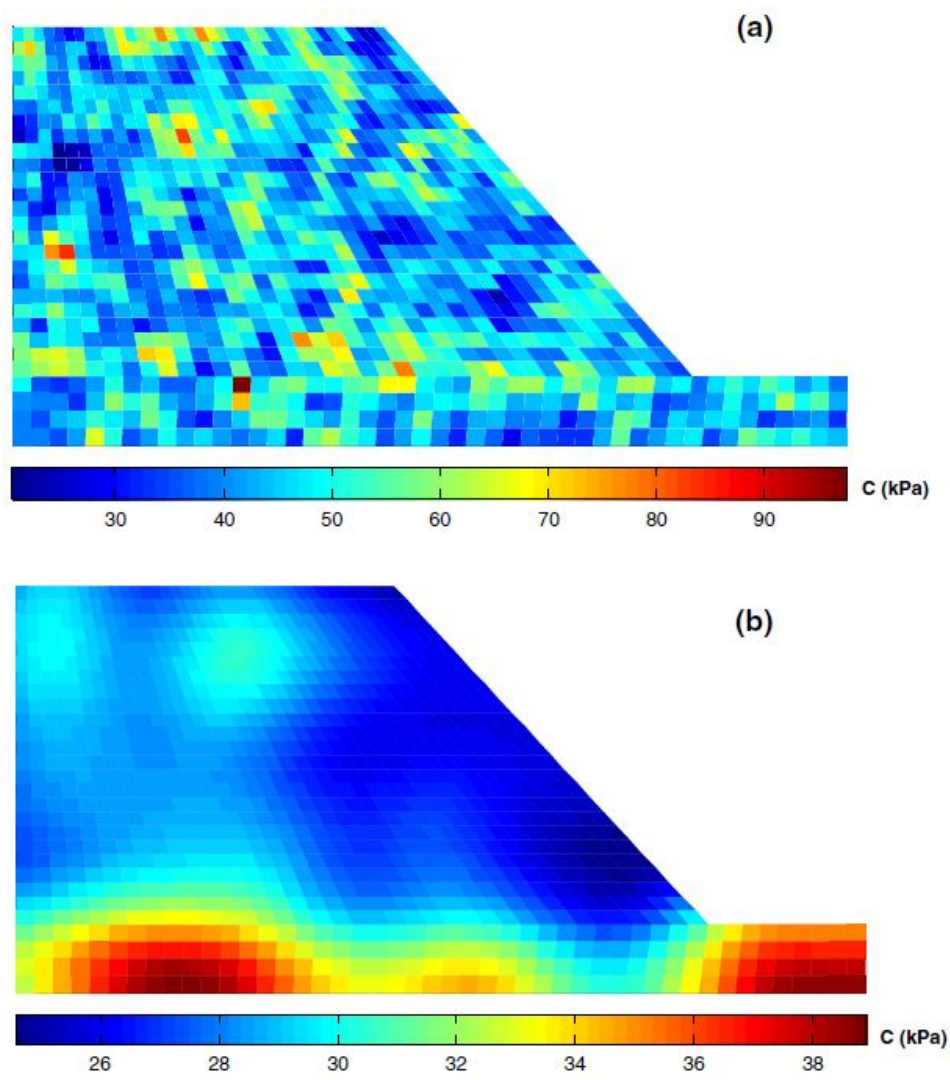

Fig. 16 Random field of the undrained shear strength with mean $45 \mathrm{kPa}$ and variance 0.3 , scales of fluctuation (a) $1 \mathrm{~m}$ and (b) 100m, Jha (2015) [57]

lognormally distributed random field. Cai et al. (2016) [12] focused on the spatial relationship between the distribution of saturated hydraulic conductivity and that of the observed pressure head in a hillslope during rainfalls. Due to the fact that the hydraulic properties usually display a high degree of spatial variability at various scales, the heterogeneity was modeled as a random field following the lognormal law. Li and Chu (2016) [72] quantitatively studied the influence of discrete element sizes of random field on the accuracy of failure probability and distribution of sliding mass volume in cohesive slope stability analyses. The spatial variability of undrained shear strength was modeled using a 2-D lognormal random field and the Cholesky decomposition was adopted to generate the random field. Li et al. (2016) [70] proposed an efficient RFEM for reliability analysis and risk assessment of soil slopes, in which the undrained shear strength of two soil layers was considered as lognormally distributed. Shen et al. (2016) [113] presented a study to simulate non-stationary fields of the particle size and permeability within a landslide soil deposit and to analyze the rainfall infiltration in random fields. Three grain size distributions of soil 
were modeled as lognormal random variables and the matrix decomposition method was adopted to generate homogeneous random fields (see Fig.17). Li et al. (2016)[74] proposed an approach for conditioning 3-D random fields based on cone penetration tests and illustrated its potential in geotechnical site exploration and cost-effective slope design through two representative examples. The soil property was first generated as an isotropic unconditional random field following a truncated Gaussian distribution using 3-D LAS method, and then transferred to conditional random field by the Kriging method. Zhu et al. (2017) [140] applied the RFEM to explore the influence of spatial variability on undrained stability of normally consolidated random slopes, where the mean strength increased linearly with depth while the coefficient of variation remained constant. The soil properties were generated as a homogeneous stationary lognormal random field across the mesh. Varkey et al. (2017) [129] studied the 3-D slope stability with spatially varying shear strength parameters by RFEM. In their study, the cohesion and friction angle were represented by the truncated Gaussian distribution.
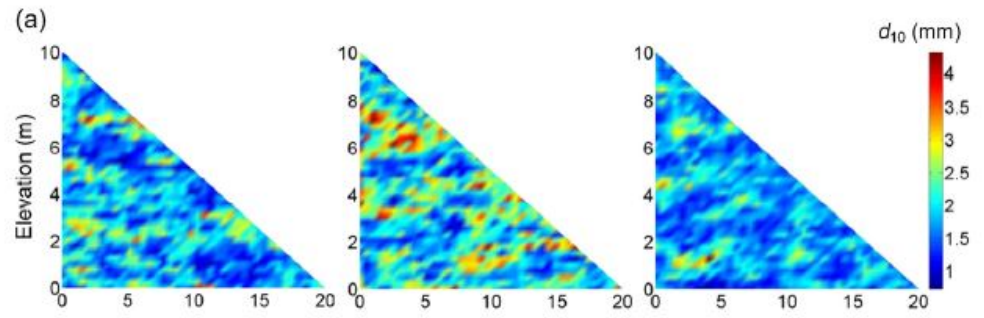

(b)
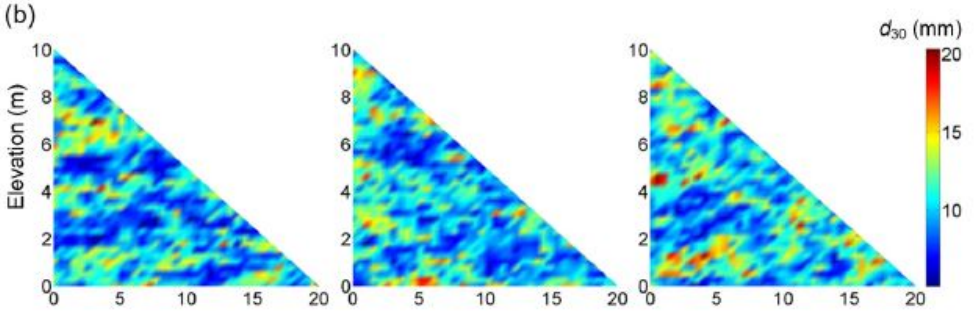

(c)
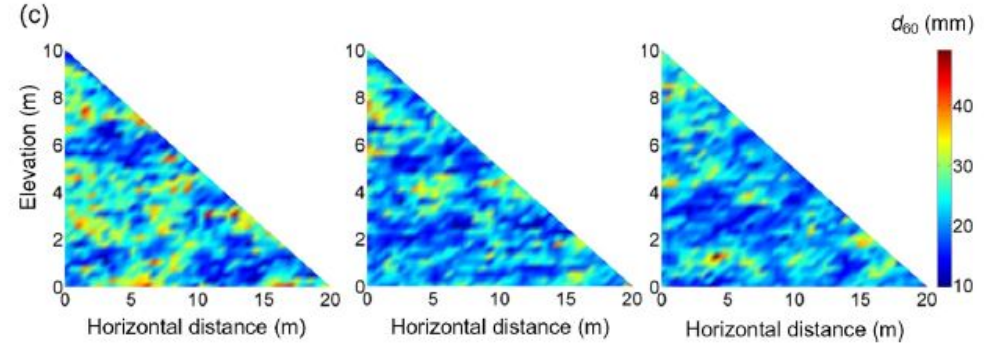

Fig. 17 Three random fields in the study of Shen et al. (2016) [113]: (a) d10; (b) d30; (c) d60 


\subsection{Settlement and consolidation analysis}

As mentioned in the last subsection, soil is a complex material that results from a combination of geologic, environmental and physicochemical processes. The variability of soil properties owing to these natural processes would lead to significant uncertainties in geotechnical engineering. For example, it would exert impacts on the bearing capacity, foundation settlement, soil consolidation, etc. Thus a well-defined local heterogeneity of soil properties is of great significance.

Fenton et al. (2002) [32] modeled the soil as random media to estimate the foundation reliability against serviceability limit state failure in the form of excessive differential settlements. The elastic modulus was generated following a lognormal distribution and the total settlement was found to be represented well by Gaussian random field. Vogel et al. (2010) [131] investigated the non-equilibrium in soil hydraulic modeling, and a heterogeneous random field with Gaussian autocovariance function was generated using the direct Fourier transform method. To illustrate the effect of correlation length on non-equilibrium dynamics, the random fields with different correlation lengths were used in horizontal direction. Rungbanaphan et al. (2010) [106] proposed a novel method for the spatial-temporal prediction of consolidation settlement based on Asaokas method. The spatial correlation structure of soil properties was introduced into the settlement prediction model, in which a 2-D stationary Gaussian random field of model parameters was produced using the spectral method. Klammler et al. (2011) [62] presented several simple approximate approaches for upscaling the cumulative distribution function of spatially correlated variables that was applied to deep foundation design. To validate these methods, a comprehensive simulation was carried out in which results of the present method were compared to these of upscaling by stochastic simulation of 10,000 random field realizations, the Gaussian random fields were generated by Cholesky decomposition with subsequent inverse normal score transformation to achieve a target. Rahman and Nguyen (2012) [102] applied a new methodology in bearing analysis. In this method the variability of soil parameters were considered within FEM by generating a Gaussian random field using LAS method. Depina et al. (2013)[18] provided an insight into the influence of soil variability on performances of wind turbine foundations subjecting to a long-term lateral cyclic loading. In their model, the soil stiffness was represented by a random field following a lognormal distribution. Paiboon et al. (2013) [94] explored influences of porosity and void sizes on effective elastic geotechnical engineering properties with a $3-\mathrm{D}$ random field model. The LAS method was adopted to generate geomaterial models containing random voids with controlled porosity and void sizes following Gaussian distribution. Bari and Shahin (2014) [5] presented a simplified probabilistic design method for ground improvement by prefabricated vertical drains considering the spatial variability of horizontal coefficient of consolidation. In their study, the variability of coefficient of horizontal consolidation was characterized as a lognormal 
distribution, which was deemed to be the most significant random variable affecting the soil consolidation.

To propagate the uncertainties of input parameters to constitutive relations of strain and stress due to the spatial variability of soil/rock mechanical parameters, Stefanos (2015) [122] conducted a stochastic finite element analysis based on the Polynomial Chaos method. In his work, the elastic module and external load were considered as second-order random fields following the lognormal distribution (see Fig. 18). Garzn et al. (2015)[35] introduced the physical modeling of soil uncertainty and presented a new approach to prepare reduced-scale soil model with controlled spatial variability. The sources and types of soil uncertainty were analyzed, and the soil intrinsic characteristics and mechanical properties following Gaussian and lognormal distributions were simulated using matrix decomposition method. To study the scale effect that the intact rock strength decreases as the specimen size increases, Goc et al. (2015)[67] proposed a continuous approach where the rock micro-scale strength properties were spatially correlated. The correlated multi-Gaussian random field was used to model the tensile strength and cohesion micro-properties of a rock mass.
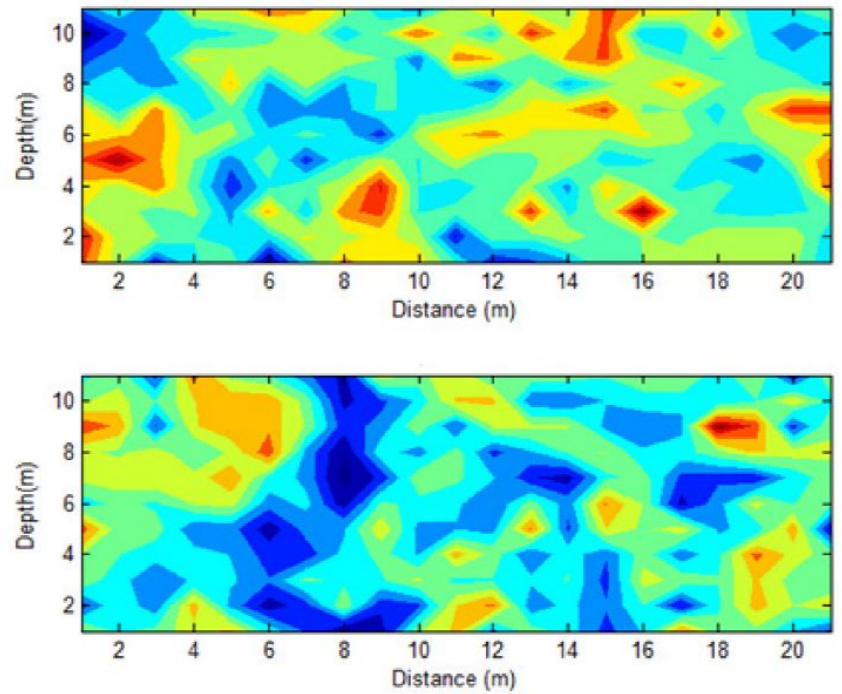

Fig. 18 Random elastic modulus fields, Stefanos (2015) [122]

Evaluation of the wave-induced soil response is vital for the design of foundations around marine structures. Zhang et al. (2016) [137] investigated the wave-induced oscillatory seabed response in a spatially random porous seabed, the spatial variability of marine soil permeability, soil shear modulus, and degree of saturation, were simulated by matrix decomposition method. As the experimental data of permeability and shear modulus were usually found to follow the lognormal distribution, all three parameters were treated to follow 
the lognormal law in simulations (see Fig. 19). Cheng et al. (2017)[15] integrated the random field simulation with numerical modeling of coupled flow and deformation to study the consolidation in spatially random unsaturated soils. The spatial variability of soil properties following the lognormal distribution, such as Young's modulus, saturated permeability, and unsaturated hydraulic parameters, were simulated using matrix decomposition method. Liu et al. (2017) [78] studied the probabilistic stability of undrained slopes using FEM and 3-D random field. In their simulation, a 3-D lognormal random field for the 3-D full model was generated.

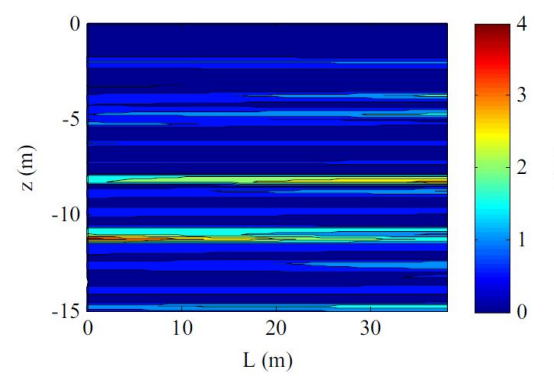

(a) $0.2 \mathrm{~m}$

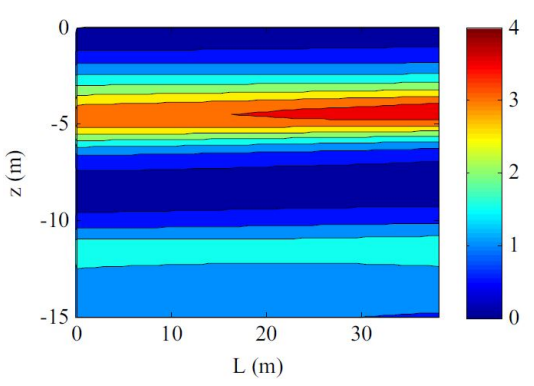

(b) $2 \mathrm{~m}$

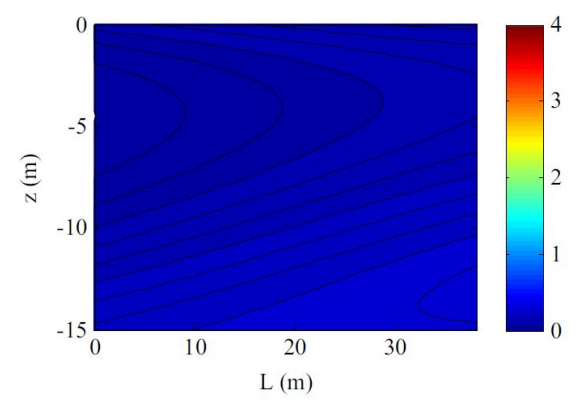

(c) $10 \mathrm{~m}$

Fig. 19 Shear modulus $G\left(\times 10^{7} \mathrm{~Pa}\right)$ of random seabed (coefficient of variation is $250 \%$ ) with correlation length of (a) $0.2 \mathrm{~m}$ (b) $2 \mathrm{~m}$ (c) $10 \mathrm{~m}$, Zhang et al. (2016) [137]

\subsection{Structural materials in civil engineering}

Structural materials from general structural steel and concrete to composite materials in civil engineering, which are commonly considered to be isotropic, intrinsically display randomness in their material properties, such as the elastic modulus, shear modulus, Poisson's ratio, etc. Since the material properties are uncertain, a random field model for the material rather than a standard deterministic model (e.g., based on average material properties) 
is more necessary to adequately account for the disorder effect. Many studies have demonstrated the effect of random material properties regarding the failure probability and reliability in civil engineering.

Shinozuka and Deodatis (1996) [115], Miguel et al. (2008) [87] applied the spectral method to simulate a multi-dimensional homogeneous Gaussian random field to model material and geometric properties of interest in structural engineering. Chen and Soares (2008) [13] proposed a spectral stochastic FEM to study laminated composite plates assuming the elastic and shear moduli as Gaussian random fields. Noh and Park (2011) [91] investigated the response variability of laminate composite plates induced by the spatial randomness of Poisson's ratio that was assumed to follow Gaussian distribution. Noh (2011) [90] also studied the plate response variability due to triple random parameters, i.e. elastic modulus, Poisson's ratio and thickness of the plate, which were assumed to follow the Gaussian distribution as well. Raabe et al. (2012) [101] presented an extension method to model the material heterogeneity for mineral subsoil machining simulations. They sampled an isotropic Gaussian random field through the fitted covariance function on equidistant grids, then each workpiece got the heterogeneity value resulted from interpolating the specific random field realization to its center by ordinary Kriging method. Dilip et al. (2013) [21] studied the impact of spatially variable resilient moduli of pavement layers by estimating the influence of variance and correlation length on pavement response to the loading. The spatial correlation was introduced into a three-layered pavement structure assuming the elastic modulus was lognormally distributed. Eliáš et al. (2013) [25] performed the stochastic discrete simulation of concrete fracture behaviors, the randomness of local material properties was merged into a discrete lattice-particle model via an autocorrelated Gaussian random field produced using KL expansion. The effect of spatial randomness on peak load and energy dissipation was investigated.

Beckmann and Hohe (2014) [7] carried out numerical analyses of uncertainties in the response of foam core sandwich structures caused by uncertain micro-structures of the core material. The random field for the foam material properties was derived and the cell size distribution was treated as lognormal law with an average cell volume $3.9 \mathrm{~mm}^{3}$ and a standard deviation of $1.6 \mathrm{~mm}^{3}$. Eliáš et al. (2015) [26] adopted the stochastic meso-level discrete model to numerically investigate the concrete fracture performed on beams of variable size and notch depth. Part of the randomness of material parameters (e.g., strength, fracture energy) introduced by finer particles and non-uniformity of the mixing process were depicted by random fields. To produce a nonGaussian random field, first Gaussian field was generated using KL expansion and expansion optimal linear estimation methods, then it was transformed to non-Gaussian fields via the isoprobabilistic transformation (see Fig. 20).

Shafei and Alipour (2015) [112] introduced the application of large-scale stochastic fields to study the spatial-temporal variability of main parameters contributing to the corrosion of concrete structures. To take probabilistic aspects of the corrosion process into account, several large-scale Gaussian and non-Gaussian random fields denoting structural characteristics, material prop- 


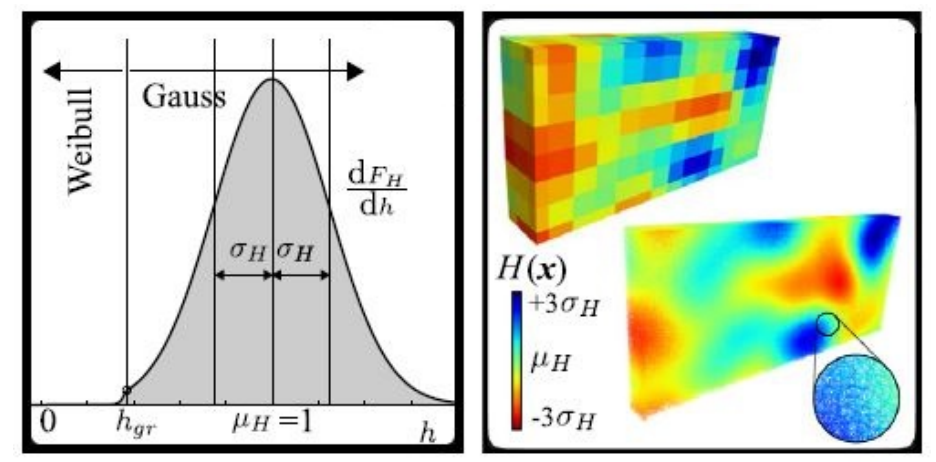

Fig. 20 Left: Weibull-Gauss probability distribution used for randomization of element parameters; Right: a random field of model parameters generated on a regular grid and projected to the model elements, Eliáš et al. (2015) [26]

erties, limit state criteria, and exposure conditions, were generated using the spectral method. Podroužek et al. (2017) [100] combined the random and gradient based fields in an attempt to capture material properties and associated variability stemming from the microstructure and macroscopic features such as the placement of coarse aggregates in random heterogeneous materials (e.g., concrete structures, infrastructure). In their study, the original particle placement scheme combined with one or more spatially variable Gaussian fields, were used to describe local fluctuations of material properties resulting from its inherent variability and construction or transport processes (see Fig. 21). Liu et al. (2017) [77] statistically evaluated the overall strength of a soil-cement column under axial compression, in which a deterministic columnar structure and a random component superimposed on the mean trend were took into consideration. The latter was simulated as a zero-mean random field following a marginal beta distribution transformed from Gaussian field.

\subsection{Turbulence flow, pipeline flow and fluid property}

Smirnov et al. (2001) [117] proposed a random flow field generation technique that can be applied to generate initial/inlet boundary in LES or particle tracking in LES/RANS simulations of turbulent flows. The technique combined advantages of the Krauchnam's method [64] and a computationally efficient implementation, in which the sample of $n$ wave-number vectors and frequencies of the modeled turbulence spectrum were assumed as Gaussian distribution. Litinova and Dolya (2011) [75] studied the numerical modeling method for fluctuations of laser radiation scattered on the reflector array in turbulent atmosphere, where the air refractive index distribution was modeled as a homogeneous, isotropic Gaussian random field with zero mean generated using shaping filter method. Javadi and El-Askary (2012) [56] performed the LES and unsteady RANS to delve into physical processes that were en- 


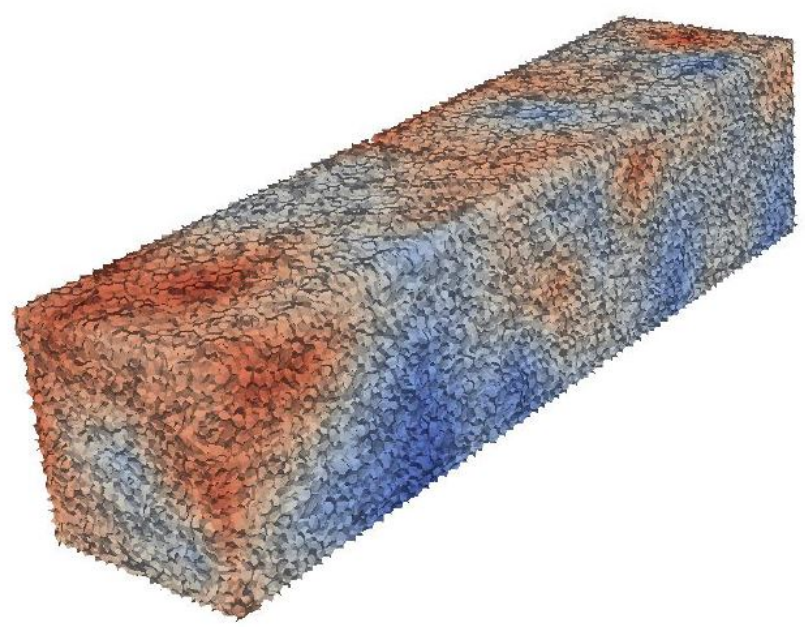

Fig. 21 A material property field modeled by Gaussian random field with a power spectral function, Podroužek et al. (2017) [100]

gendered in the injection of a circular synthetic jet in a zero pressure gradient turbulent boundary layer. The physical realism of the incoming turbulent boundary layer was secured using the same random field generation method as Smirnov et al. (2001) [117]. To do the pipe system diagnosis, Massari et al. (2014)[84] proposed a stochastic successive linear estimator algorithm to detect extended partial blockages in pipelines via pressure transient measurements. In their study, the diameter distribution resulting from the formation of blockages was assumed to follow a lognormal law and generated using the FFT method proposed by Gutjahr (1989)[47]. Noetinger et al. (2018) [89] put forward an approach to estimate the effective viscosity of a random mixture of Newtonian fluids (e.g., suspension, emulsion) that ignored capillary effects. The key idea was to assume the mixture can be represented by a single fluid characterized by a local viscosity modeled by a position-dependent stationary random function. The random function can be described by its two first moments: local average and two-point correlation function, where the latter was considered as a multi-Gaussian variable following a normal (simplest case) or lognoraml distribution (see Fig. 22). In their work, the random viscosity fields were produced by the $3-\mathrm{D}$ version of FFT-MA method.

\subsection{Image segmentation or generation}

In image analysis and computer vision, image segmentation is a crucial task which aims to divide a given image into several non-overlapping regions based on statistical similarities such as means, distributions and structure tensors. Schluter et al. (2010) [110] generated a multi-Gaussian random field of soil porosity by direct Fourier transform method as an artificial test im- 

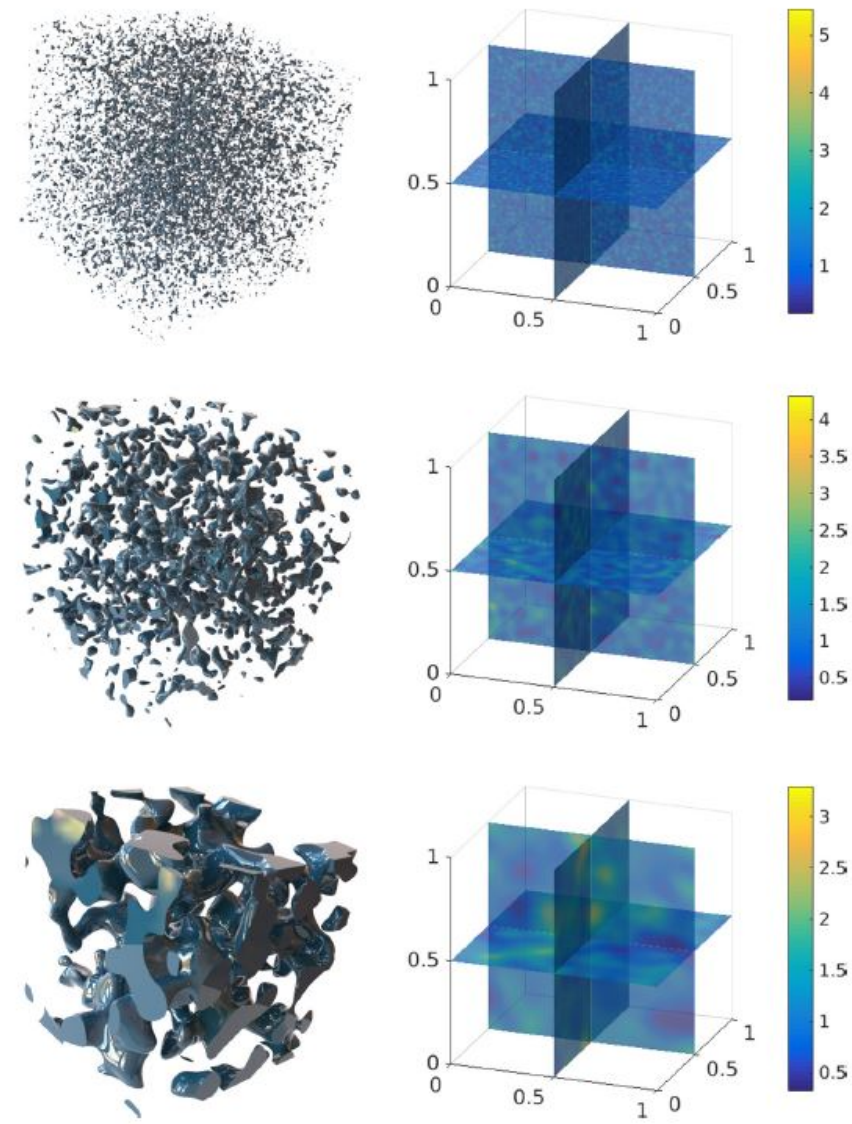

Fig. 22 Isolevel surfaces and slices of random viscosity field (variance is 0.1 , correlation length is $3 \delta_{x}, 10 \delta_{x}$, and $25 \delta_{x}$ at resolution $257^{3}$, top to bottom), Noetinger et al. (2018) [89]

age to evaluate the robustness of their proposed segmentation method for the segmentation of X-ray micro-tomography images. In remote sensing hyperspectral image processing, Veganzones and Hernandez (2010) [130] proposed a novel hybrid validation method composing of a simulation module which generates validation images from stochastic models. In their method, the synthetic ground-truth abundance was simulated using the method proposed by Kozintsev (1999) [63] for efficient generations of Gaussian random field (see Fig. 23).

\subsection{Earthquake}

Images of the spatial-temporal evolution of earthquake slip on fault planes provide compelling evidence that fault displacement is spatially variable at 

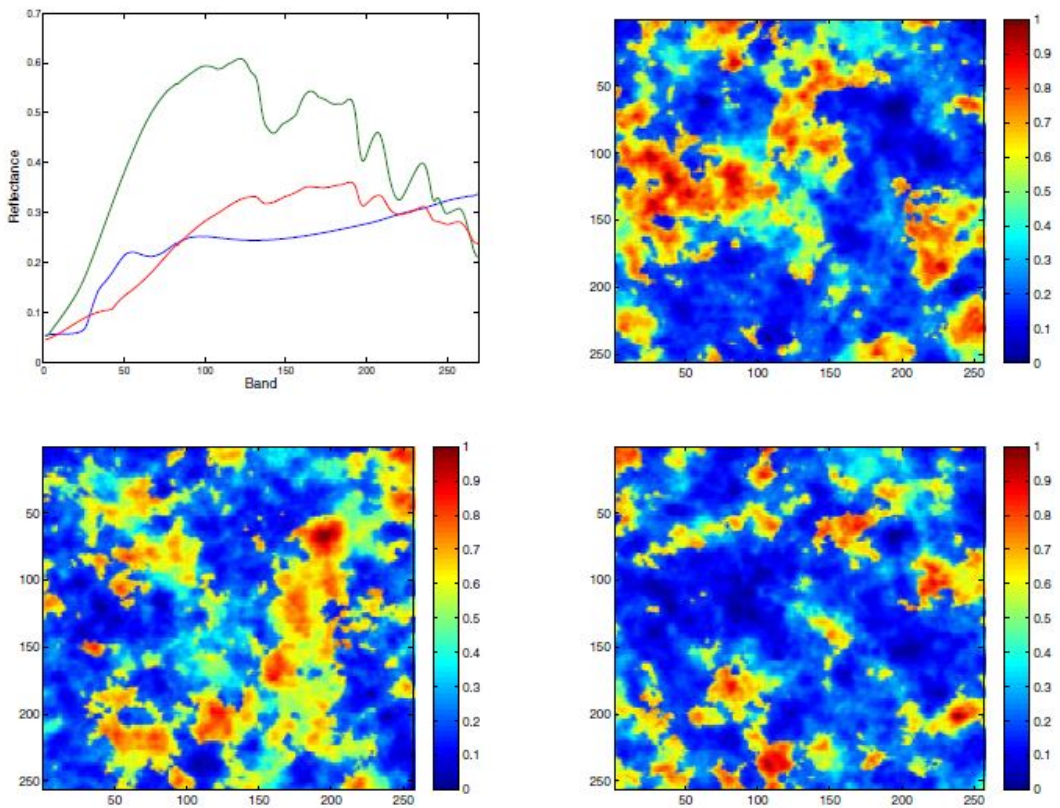

Fig. 23 Example of endmembers (constituent spectra of the materials in the image) and abundance images used to generate the synthetic hyperspectral image. This example corresponds to a 10-endmembers dataset image using 3 endmembers, Veganzones and Hernandez (2010)

[130]

all resolvable scales. Mai and Beroza (2002) [80] developed a stochastic characterization of the earthquake slip complexity, they investigated the validity of Gaussian, exponential, von Karman, and fractal autocorrelation models by considering 44 slip distributions for worldwide crustal earthquakes (see Fig. 24). In their work, random field model parameters (e.g., fractal dimension, correlation length) were combined with macroscopic earthquake source parameters such as moment magnitude and fault length/width. For the same seismic event, Lavorato et al. (2017) [66] proposed two methods to generate earthquake asynchronous signals at different space points. The signal frequency was computed by considering a Gaussian distribution of the signal amplitude at each point.

\subsection{Others}

(1) Rainfall intensity in hydrology. Gyasi-Agyei and Pegram (2014) [48] developed a method for interpolating the daily rainfall networks using simulated radar fields for realistic hydrological modeling of spatial rain field ensembles. The Gaussian random fields were generated by sampling from correlogram parameters conditioned on the Gauge Wetness Ratio and then these 


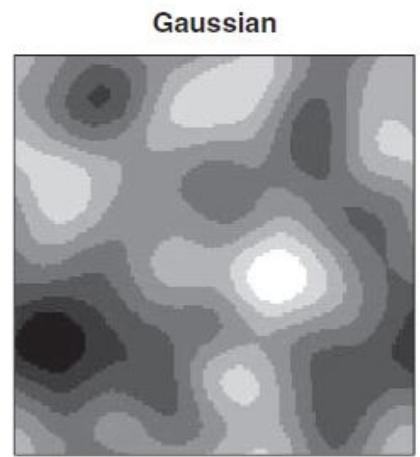

von Karman

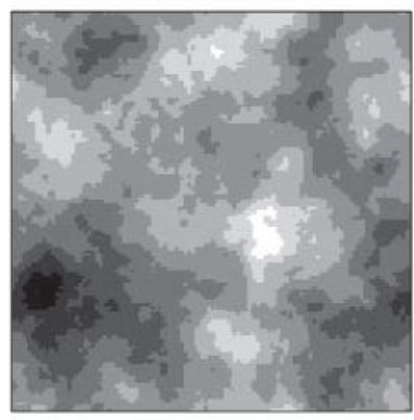

Exponential

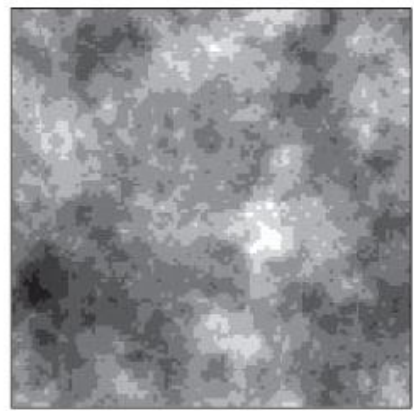

Fractal

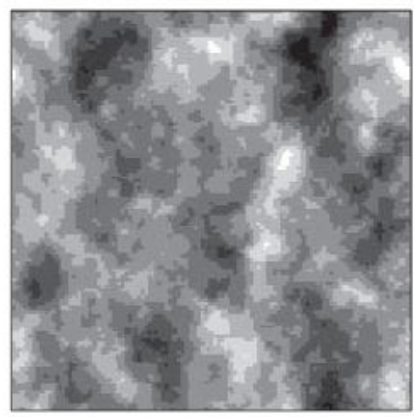

Fig. 24 Random field models generated with same phasing to facilitate the comparison. The grid dimensions are $30 \mathrm{~km} \times 30 \mathrm{~km}$ and the correlation length is $5 \mathrm{~km}$, Mai and Beroza (2002) [80]

fields were conditionally merged to the gauge observations transformed into the Gaussian domain. The joint sequential simulation of multi-Gaussian field method was adopted to calculate unconditional Gaussian random fields using the $\mathrm{R}$ package gstat.

(2) Detection of deep-water oil leak. Prior to the oil accident of the Deepwater Horizon, the presence of oil in the sea is always confined at shallowwater owing to either natural processes or human-induced pollution. Now there is a need to quantify the oil settled in the water column and determine its physical properties to assist the oil recovery. Xenaki et al. (2013)[132] proposed a high-frequency acoustic method to localize the oil polluted area and characterize the parameters of its spatial covariance. In their method, the physical model for the submerged oil was represented as a Gaussian random field of compressibility fluctuations which exhibited stationary spatial correlation, and the random field generator based on the FFT-MA approach was introduced to implement the physical model.

(3) Material processing. In construction industry, the core drilling with diamond impregnated tools is a widely-used method. Various components of 
both the machined material and the tool usually result in complex processes. Herbrandt et al. (2018) [50] presented a numerical model to simulate the normal force arising during a grinding process in cement for single diamond grinding. Due to the inhomogeneity of scratched mineral subsoils, the normal force was expected to vary at different positions of the workpiece. This heterogeneous nature was taken into account by sampling from a Gaussian random field in their study.

(4) Tunnel stability analysis. With the increase of the tunnel size, the tunnel stability becomes affected more and more by the spatial variability of rock properties. Thus it has become more important to evaluate effects of the spatial variability of rock properties. Song et al. (2011) [119] performed a stochastic numerical analysis based on finite difference method to explore the influence of spatially variable rock properties on tunnel behaviors, e.g., elasticplastic interface, deformation, ground reaction curve, and failure mechanism, etc. A truncated Gaussian random field generated by TBM was adopted to simulate the randomness of rock properties. Pan and Dias (2017) [95] carried out the probabilistic analysis of the tunnel face stability in spatially random soils using sparse Polynomial Chaos expansion, in which the cohesion, friction angle, and unit weight were considered as lognormal random fields.

\section{Software and package}

With the development of random field theory and recent advances in numerical techniques, computer, visualization and image processing, the corresponding computer codes have been developed by researchers and engineers for the random field generation, which includes the in-house code or opensource code, independent package or general software that combined with the random field generation as a module, etc. To make the Gaussian random field generation methods presented in Section 2 more easily be understood and implemented, in this Section, several software and packages related to the random field generation which are freely obtained from the Internet are briefly introduced. It should be noted that for brevity we only present general information of these software and packages without any detailed user guide and instructions, readers of interest can refer to the given website or references for more details.

\subsection{RFEM}

As mentioned is Section 3, Fenton and Griffiths (1993) [30] proposed a random finite element method (RFEM) that combines the random field simulation with the finite element method, which has been widely applied to study random behaviors of soils/rocks in the context of a variety of classical geotechnical problems, ranging from settlement of shallow foundations to the collapse of rock pillars, etc. Corresponding RFEM software was also developed and 
is freely available on the Web. RFEM software was programmed using the Fortran 77 language, and the complete current Version 1.1.2 for the Windows environment has been released since 2012. To visualize the outputs of these programs, users have to install Postscript viewers such as the Ghostscript and Ghostview. Within the distribution at http://random.engmath.dal.ca/ $\mathrm{rfem} /$, the folder 'utils' presents a series of programs to compute the random field information including local average variances, random variables, normal distribution probabilities, etc. Readers of interest can refer to [33,118] for full descriptions of RFEM software and to http://random.engmath.dal.ca/ $\mathrm{rfem} /$ for the source code and revision history. Below two figures illustrate an example for RFEM software (see Figs. 25, 26).

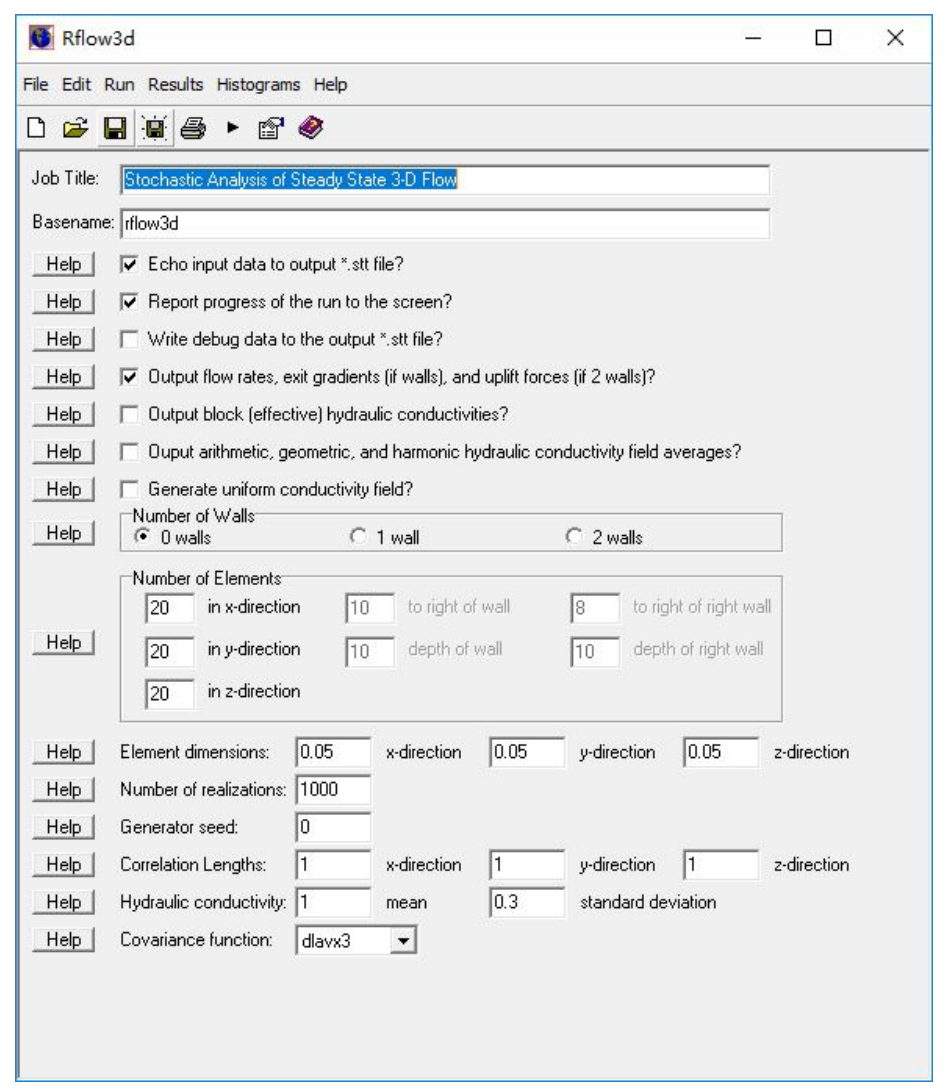

Fig. 25 Main window of Rflow3d in RFEM software 


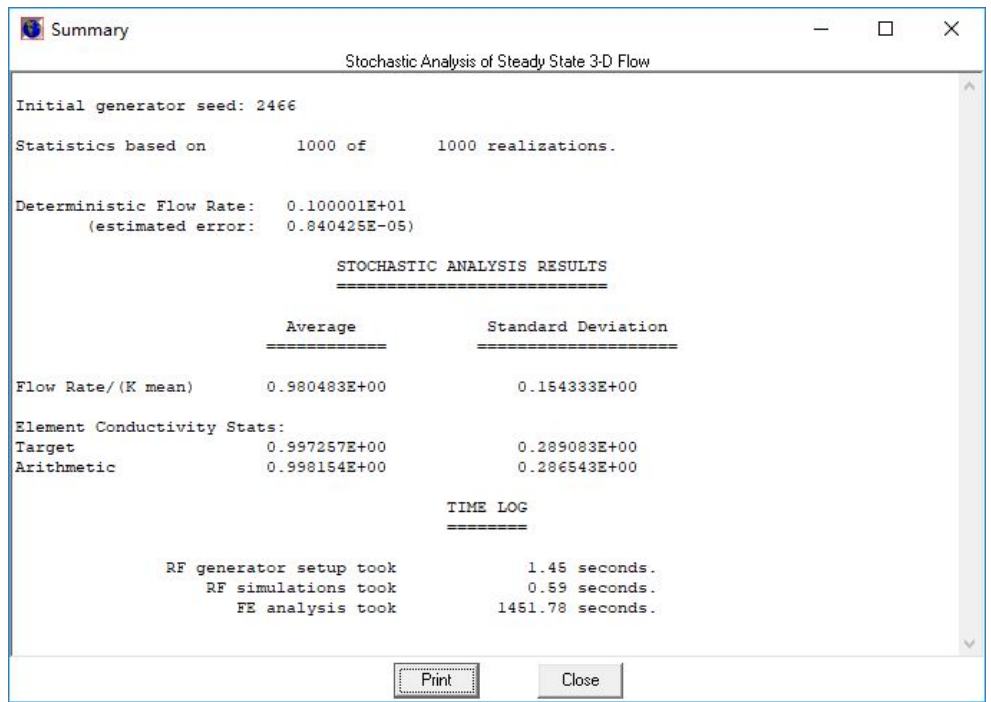

Fig. 26 Results summary of RFEM software

\subsection{SGeMS}

The Stanford Geostatistical Modeling Software (SGeMS) is an open-source software developed at Stanford University, it is capable of performing several geostatistics algorithms to model earth systems and more generally space-time distributed phenomena. In SGeMS, an user-friendly interface (see Fig. 27), an interactive 3-D visualization (see Fig. 28), and a wide selection of algorithms are provided for geostatistics practitioners. For more complex tasks, users can use the embedded Python scripting language and SGeMS plug-in mechanism to develop new algorithms. In SGeMS, there are many methods available for the random field generation, such as Kriging method, sequential Gaussian simulation, etc. SGeMS is available on both Linux and Microsoft Windows platforms, the source code and a Microsoft Windows executable are distributed on the website http://sgems.sourceforge.net. Just like RFEM software, some third-party libraries are required to compile SGeMS. Readers of interest are referred to the book [121] for more details on the step-by-step guide to use SGeMS algorithms, and to get the fundamental theory, implementation demonstrations, etc.

\subsection{RSG}

Surface roughness is a measure of topographic height variations of the surface. Rough surfaces are commonly classified into the subcategories of deterministic and random surfaces, where random surfaces are stochastic and are usually characterized by random fields. Bergstrm [9] developed a Random 


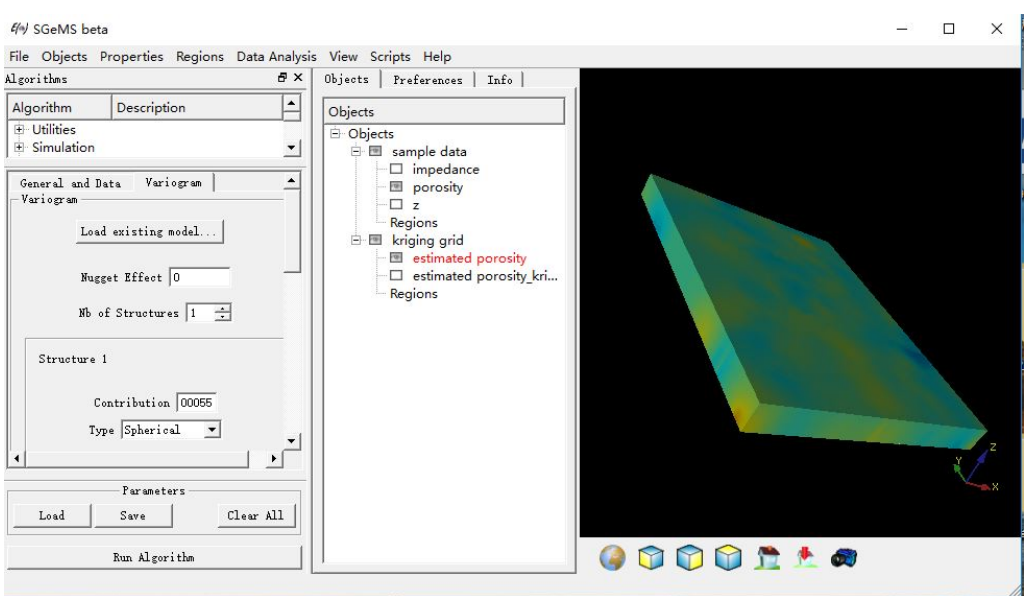

Fig. 27 SGeMS main window

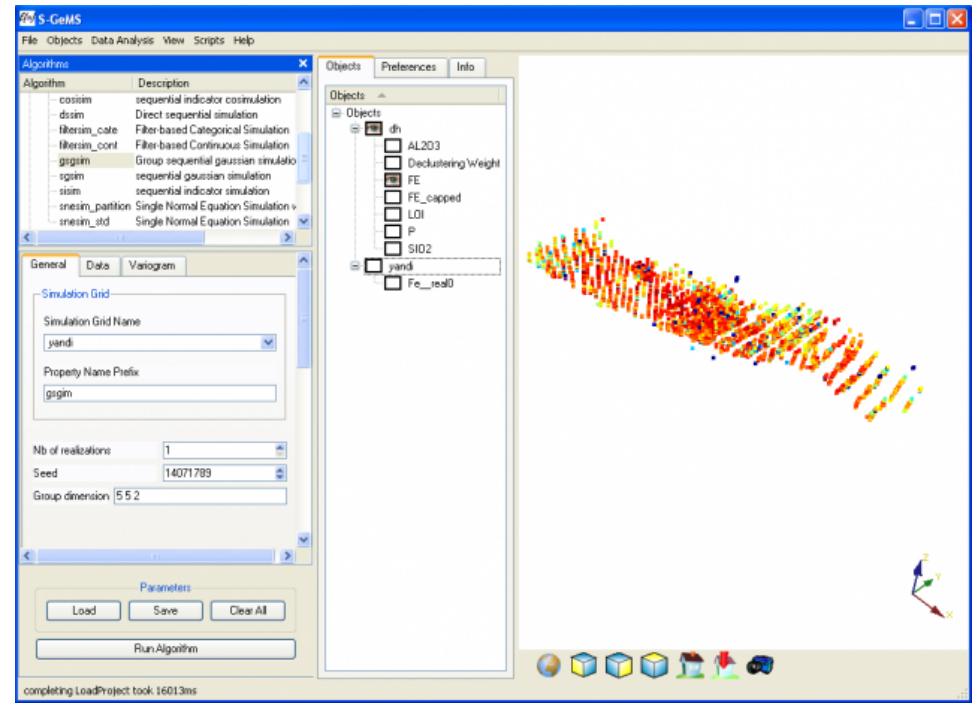

Fig. 28 SGeMS Visualizing a point set, http://sgems.sourceforge.net/

Surface Generation (RSG) software to generate the random rough surfaces with Gaussian distribution using the MA method. Programmed in Matlab, RSG provides GUI for generation and analysis of 1-D and 2-D random rough surfaces. It contains two main parts, the first part is the random rough surface generator of 1-D profiles and 2-D (isotropic and anisotropic) square surfaces following Gaussian distribution; the second part is the surface analysis, in this part the autocovariance function and correlation length of 1-D profile and 2-D surface can be computed, the height distribution function and the RMS height can be obtained as well. It is worth pointing out although RSG is designed 
to generate 1-D or 2-D random rough surfaces, it is easy to be extended for 3 -D or other random fields generation according to users' requirements. Readers of interest can refer to the web at http://www.mysimlabs.com/surface_ generation.html for more details and download the source code. Below figures display the GUI of RSG and the generated Gaussian random field (see Figs. 29, 30).

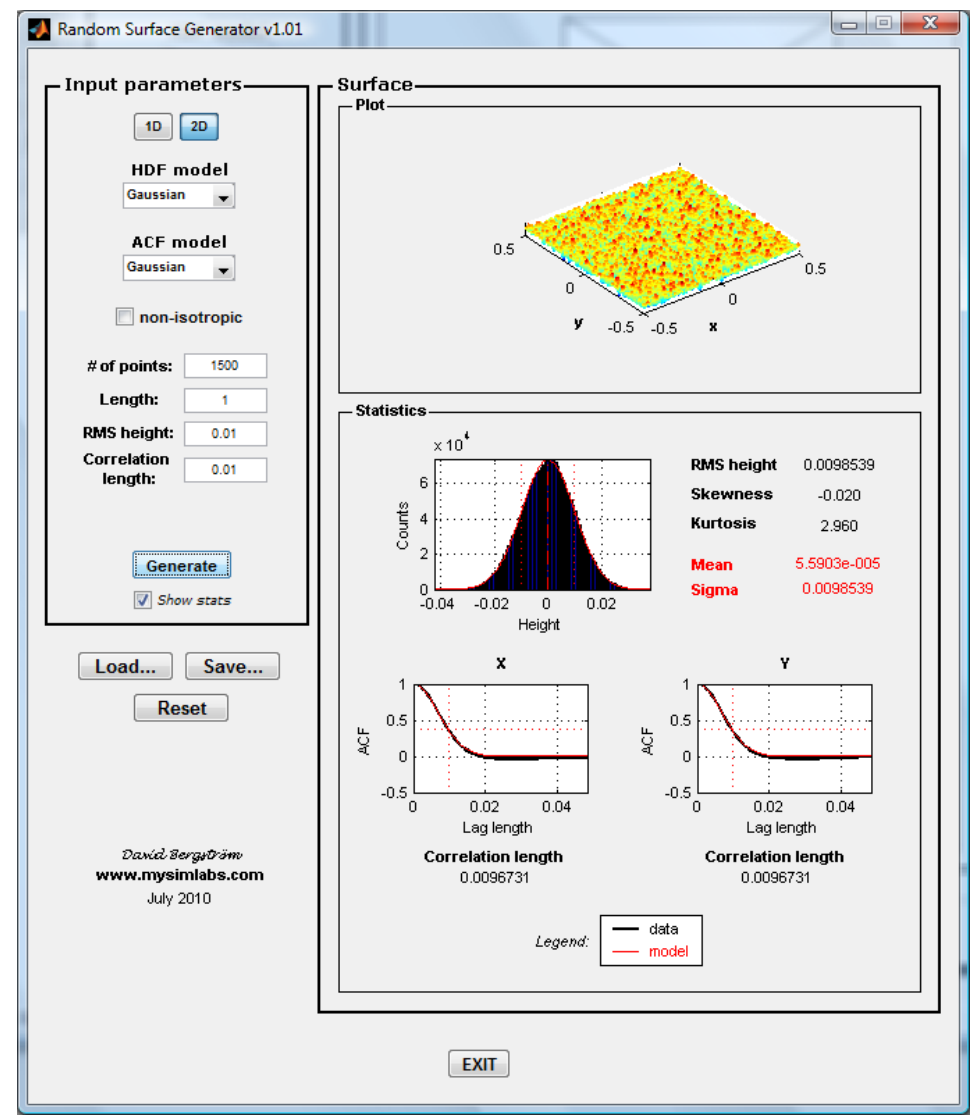

Fig. 29 GUI of RGS software

\subsection{Others}

(1) $R$ packages: RandomFields, gstat. $R$ is a programming language and free software environment for statistical computing and graphics. It has been applied widely among statisticians and data miners to develop statistical software and data analysis. In $\mathrm{R}$ studio, the package RandomFields for the inference on Gaussian field simulation is provided, as well as methods for 

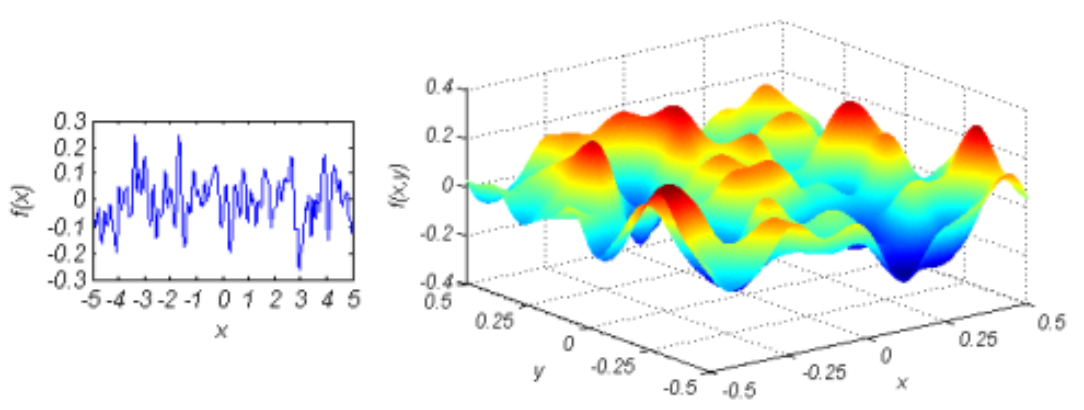

Fig. 30 Matlab plots of Gaussian random rough surfaces generated by RSG. Left: surface profile using 'rsgeng1D' function. Right: 2-D surface using 'rsgeng2D' function

the simulation of extreme value random fields. The newest version 3.1.50 of RandomFields was released by Schlather et al. (2017) [108] and readers of interest can refer to https://CRAN.R-project.org/package=RandomFields for more details. The package gstat includes the functions: variogram modeling; simple, ordinary and universal point or block kriging; spatio-temporal kriging; sequential Gaussian or indicator simulation; variogram and variogram map plotting utility functions. The latest version 2.0-0 of gstat was released by Pebesma et al. (2018) [98] and readers of interest are referred to https:// cran.r-project.org/web/packages/gstat/index.html for more details.

(2) IGW. Interactive Groundwater (IGW) is a software environment for real-time 2-D and 3-D unified deterministic and stochastic groundwater modeling developed by Li and Liu [73]. It can be used to explore: visually creating an aquifer of desired configurations and then immediately investigating and visualizing the groundwater system. IGW can handle the stochastic modeling including interactive and visual unconditional/conditional simulation of hydrogeochemical properties, exploratory data analysis, built-in variogram modeling with automatic/manual fitting, interactive spatial interpolation, regression, smoothing/filtering, multi-scale Kriging and random field generation, conditional MCS, etc. The programming language of IGW includes Visual Basic 6, FORTRAN 5, Borland C++ Builder 6 and Delphi 5. The 3-D verison IGW4.7 was released in 2006 according to the website information. Readers of interest can refer to [73] and the website https://www.egr.msu.edu/ lishug/ research $\backslash \% 20$ web/NSF-CRCD-IGW.htm for more details.

(3) GenPack. To solve uncertainty-related issues in reservoir simulations, a general package (GenPack) was developed by Xue et al. (2017) [133]. In Genpack, three basic functions required for stochastic analyses in petroleum engineering are built up: the generation of random parameter fields, the stochastic forwarding modeling to quantify the predictive uncertainty, and the automatic history matching to calibrate the numerical model against the sequentially available data. For random field generation in GenPack, two Gaussian random field generation methods are provided: sequential Gaussian simulation and KLexpansion. In addition, GenPack was developed in a modularized manner and 
can be easily integrated with existing commercial reservoir simulator to facilitate its application, and can leverage parallel computing. It is programmed using the $\mathrm{C}++$ language and is freely available at the website https://www . researchgate.net/publication/313114201_GenPack_Code. Readers of interest can refer to [133] for more details.

\section{Summary}

In nature and engineering, a large body of materials and physical processes show uncertainties in their properties and behaviors on spatial and temporal spaces. Generation of random fields to model the uncertainties is a significant task in stochastic-related numerical analyses. Due to the fundamental role of Gaussian random field in modeling the uncertainties in scientific and engineering problems, this paper presents a comprehensive review on state-of-the-art Gaussian random field generation methods and its applications of engineering interest. We briefly introduce basic mathematical concepts in Gaussian random field, systematically present seven popular Gaussian random generation methods from the perspective of basic idea and mathematical derivation, and thoroughly summarize the characteristics of these generation methods. We also present several representative applications of Gaussian random field in various scientific and engineering issues of interest in recent two decades. Finally, for the ease of implementation of Gaussian random field generation methods, relevant up-to-date open-source software and packages freely available from the Internet are briefly introduced.

It should be noted that the reference list in this review is by no means exhaustive, only widely-used generation methods and representative applications and software are reviewed. Nevertheless, this review constitutes a comprehensive collection of classical and popular methods for generating Gaussian random field including TBM, spectral method, matrix decomposition method, KL expansion, MA method, sequential simulation, and LAS, and a broad class of applications in scientific and engineering problems such as subsurface flow and transport, slope stability, turbulent flow, earthquake, image segmentation, etc, as well as several relevant up-to-date open-source software and packages for Gaussian random field generation. Considering their importance, these methods, applications and software are selected to be reviewed in order to benefit scientists and engineers who are conducting research related with stochastic modeling.

Acknowledgements The authors gratefully acknowledge the support from the National Natural Science Foundation of China (No. 51874262) and the Research Funding from King Abdullah University of Science and Technology (KAUST) through the grants BAS/1/135101-01. We also gratefully acknowledge the papers that contributing to our work, and the figures that we reprinted from these papers, textbooks and web resources. The authors would like to thank the anonymous reviewers for their helpful and constructive comments that greatly contributed to improving the overall quality of the paper. 


\section{References}

1. Abrahamsen, P.: A review of gaussian random fields and correlation functions (1997)

2. Adler, R.J., Taylor, J.E.: Random fields and geometry. Springer Science \& Business Media (2009)

3. Alkhatib, A.: Applying the multi-level monte carlo method to quantify uncertainty for chemical eor processes. In: Second EAGE Integrated Reservoir Modelling Conference (2014)

4. Bachmayr, M., Cohen, A., Migliorati, G.: Representations of gaussian random fields and approximation of elliptic pdes with lognormal coefficients. J. Fourier Anal. Appl. 24(3), 621-649 (2018)

5. Bari, M.W., Shahin, M.A.: Probabilistic design of ground improvement by vertical drains for soil of spatially variable coefficient of consolidation. Geotext. Geomembr. 42(1), 1-14 (2014)

6. Beaudoin, A., De Dreuzy, J.R., Erhel, J., Pichot, G.: Convergence analysis of macro spreading in 3d heterogeneous porous media 41, 59-76 (2013)

7. Beckmann, C., Hohe, J.: Effects of material uncertainty in the structural response of metal foam core sandwich beams. Compos. Struct. 113(1), 382-395 (2014)

8. Benson, D.A., Meerschaert, M.M., Revielle, J.: Fractional calculus in hydrologic modeling: A numerical perspective. Adv. Water Resour. 51, 479-497 (2013)

9. Bergström, D., Powell, J., Kaplan, A.: A ray-tracing analysis of the absorption of light by smooth and rough metal surfaces. J. Appl. Phys. 101(11), 113,504 (2007)

10. Blanchard, P., Coulaud, O., Darve, E.: Fast hierarchical algorithms for generating gaussian random fields. Ph.D. thesis, Inria Bordeaux Sud-Ouest (2015)

11. Boschan, A., Nœtinger, B.: Scale Dependence of Effective Hydraulic Conductivity Distributions in 3D Heterogeneous Media: A Numerical Study. Transp. Porous Media 94(1), 101-121 (2012)

12. Cai, J.s., Yan, E.c., Yeh, T.c.J., Zha, Y.y.: Effects of heterogeneity distribution on hillslope stability during rainfalls. Water Sci. Eng. 9(2), 134-144 (2016)

13. Chen, N.Z., Soares, C.G.: Spectral stochastic finite element analysis for laminated composite plates. Comput. Methods Appl. Mech. Eng. 197(51-52), 4830-4839 (2008)

14. Chen, X., Liu, J., Xie, N., Sun, H.: Probabilistic analysis of embankment slope stability in frozen ground regions based on random finite element method. Sci. Cold Arid Reg. 7(4), 0354-0364 (2015)

15. Cheng, Y., Zhang, L., Li, J., Zhang, L.M., Wang, J., Wang, D.: Consolidation in spatially random unsaturated soils based on coupled flow-deformation simulation. Int. J. Numer. Anal. Methods Geomech. 41(5), 682-706 (2017)

16. Christakos, G.: Random field models in earth sciences. Courier Corporation (2012)

17. Davis, M.W.: Production of conditional simulations via the LU triangular decomposition of the covariance matrix. Math. Geol. 19(2), 91-98 (1987)

18. Depina, I., Le, T., Eiksund, G., Benz, T.: Cyclic behavior of laterally loaded piles in soils with variable properties. Proceedings of the International Offshore and Polar Engineering Conference 9, 583-588 (2013)

19. Dietrich, C.R., Newsam, G.N.: Efficient generation of conditional simulations by chebyshev matrix polynomial approximations to the symmetric square root of the covariance matrix. Math. Geol. 27(2), 207-228 (1995)

20. Dietrich, C.R., Newsam, G.N.: Fast and Exact Simulation of Stationary Gaussian Processes through Circulant Embedding of the Covariance Matrix. SIAM J. Sci. Comput. 18(4), 1088-1107 (1997)

21. Dilip, D.M., Sivakumar Babu, G.: Influence of spatial variability on pavement responses using latin hypercube sampling on two-dimensional random fields. J. Mater. Civ. Eng. 26(11), 04014,083 (2013)

22. Dimitrakopoulos, R., Luo, X.: Generalized sequential gaussian simulation on group size $\nu$ and screen-effect approximations for large field simulations. Math. Geol. 36(5), 567-591 (2004)

23. Drakos, S., Pande, G.: Stochastic finite element analysis for transport phenomena in geomechanics using polynomial chaos. Global J. Res. Eng. E: Civ. Struct. (2015) 
24. Van den Eijnden, A., Hicks, M.: Conditional simulation for characterizing the spatial variability of sand state. In: Proc. 2nd Int. Symp. Comp. Geomech., Croatia, pp. 288-296 (2011)

25. Eliáš, J., Vořechovskỳ, M., Le, J.L.: Lattice modeling of concrete fracture including material spatial randomness. Eng. Mech. 20, 413-426 (2013)

26. Eliáš, J., Vořechovskỳ, M., Skoček, J., Bažant, Z.P.: Stochastic discrete meso-scale simulations of concrete fracture: Comparison to experimental data. Eng. Fract. Mech. 135, 1-16 (2015)

27. Emery, X., Furrer, R., Porcu, E.: A turning bands method for simulating isotropic Gaussian random fields on the sphere. Stat. Probab. Lett. (2018)

28. Emery, X., Lantuéjoul, C.: TBSIM: A computer program for conditional simulation of three-dimensional Gaussian random fields via the turning bands method. Comput. Geosci. 32(10), 1615-1628 (2006)

29. Feischl, M., Kuo, F.Y., Sloan, I.H.: Fast random field generation with h-matrices. Numer. Math. 140(3), 639-676 (2018)

30. Fenton, G.A., Griffiths, D.: Statistics of block conductivity through a simple bounded stochastic medium. Water Resour. Res. 29(6), 1825-1830 (1993)

31. Fenton, G.A., Griffiths, D.: Random Field Generation and the Local Average Subdivision Method. In: Probabilistic Methods in Geotechnical Engineering, pp. 201-223. Springer, Vienna (2007)

32. Fenton, G.a., Griffiths, D.V.: Probabilistic Foundation Settlement on Spatially Random Soil. J. Geotech. Geoenviron. Eng. 128(5), 381-390 (2002)

33. Fenton, G.A., Griffiths, D.V.: Risk assessment in geotechnical engineering, vol. 461. Wiley Online Library, New York (2008)

34. Fenton, G.A., Vanmarcke, E.H.: Simulation of Random Fields via Local Average Subdivision. J. Eng. Mech. 116(8), 1733-1749 (1990)

35. Garzón, L.X., Caicedo, B., Sánchez-Silva, M., Phoon, K.K.: Physical modelling of soil uncertainty. Int. J. Phys. Model. Geotech. 15(1), 19-34 (2015)

36. Ghanem, R.G., Spanos, P.D.: Stochastic finite element method: Response statistics In: Stochastic Finite Elements: A Spectral Approach, pp. 101-119. Springer (1991)

37. Goda, K., Yasuda, T., Mori, N., Mai, P.M.: Variability of tsunami inundation footprints considering stochastic scenarios based on a single rupture model: application to the 2011 tohoku earthquake. J. Geophys. Res. Oceans 120(6), 4552-4575 (2015)

38. Gómez-Hernández, J.J., Journel, A.G.: Joint sequential simulation of multigaussian fields. In: Geostatistics Troia92, pp. 85-94. Springer (1993)

39. Graham, I.G., Kuo, F.Y., Nichols, J.A., Scheichl, R., Schwab, C., Sloan, I.H.: Quasimonte carlo finite element methods for elliptic pdes with lognormal random coefficients. Numer. Math. 131(2), 329-368 (2015)

40. Graham, I.G., Kuo, F.Y., Nuyens, D., Scheichl, R., Sloan, I.H.: Analysis of Circulant Embedding Methods for Sampling Stationary Random Fields. SIAM J. Numer. Anal. 56(3), 1871-1895 (2018)

41. Griffiths, D., Fenton, G.A.: Probabilistic slope stability analysis by finite elements Journal of Geotechnical and Geoenvironmental Engineering 130(5), 507-518 (2004)

42. Griffiths, D., Huang, J., Fenton, G.: Probabilistic slope stability analysis using rfem with non-stationary random fields. Risk V, Schweckendiek T, van Tol AF, Pereboom D et al (eds) Geotechnical Safety pp. 704-709 (2015)

43. Griffiths, D., Paiboon, J., Huang, J., Fenton, G.: Numerical analysis of the influence of porosity and void size on soil stiffness using random fields. Comput. Methods Geomech.: Front. New Appli. 1, 21-27 (2011)

44. Griffiths, D.V., Huang, J., Fenton, G.A.: Influence of Spatial Variability on Slope Reliability Using 2-D Random Fields. J. Geotech. Geoenviron. Eng. 135(10), 1367-1378 (2009)

45. Guo, Z., Brusseau, M.L.: The impact of well-field configuration on contaminant mass removal and plume persistence for homogeneous versus layered systems. Hydrol. Process. 31(26), 4748-4756 (2017)

46. Gutjahr, A., Bullard, B., Hatch, S.: General Joint Conditional Simulations Using a Fast Fourier Transform Method. Math. Geol. 29(3), 361-389 (1997) 
47. Gutjahr, A.L.: Fast fourier transforms for random field generation: Project report for los alamos grant to new mexico tech. Ph.D. thesis, New Mexico Institute of Mining and Technology (1989)

48. Gyasi-Agyei, Y., Pegram, G.: Interpolation of daily rainfall networks using simulated radar fields for realistic hydrological modelling of spatial rain field ensembles. J. Hydrol. 519(PA), 777-791 (2014)

49. Hackbusch, W.: Hierarchical matrices: algorithms and analysis, vol. 49. Springer (2015)

50. Herbrandt, S., Ligges, U., Ferreira, M.P., Kansteiner, M., Biermann, D., Tillmann, W., Weihs, C.: Model based optimization of a statistical simulation model for single diamond grinding. Comput. Stat. 33(3), 1127-1143 (2018)

51. Hicks, M.A., Samy, K.: Influence of heterogeneity on undrained clay slope stability. Q. J. Eng. Geol. Hydrogeol. 35(1), 41-49 (2002)

52. Huang, J., Griffiths, D.V.: Determining an appropriate finite element size for modelling the strength of undrained random soils. Comput. Geotech. 69, 506-513 (2015)

53. Hunger, L., Cosenza, B., Kimeswenger, S., Fahringer, T.: Random fields generation on the GPU with the spectral turning bands method. Lecture Notes in Computer Science (including subseries Lecture Notes in Artificial Intelligence and Lecture Notes in Bioinformatics) $\mathbf{8 6 3 2}$ LNCS, 656-667 (2014)

54. Hunger, L., Cosenza, B., Kimeswenger, S., Fahringer, T.: Spectral turning bands for efficient Gaussian random fields generation on GPUs and accelerators. Concurr. Comput. 27(16), 4122-4136 (2015)

55. Jamshidi Chenari, R., Alaie, R.: Effects of anisotropy in correlation structure on the stability of an undrained clay slope. Georisk 9(2), 109-123 (2015)

56. Javadi, A., El-Askary, W.A.: Numerical prediction of turbulent flow structure generated by a synthetic cross-jet into a turbulent boundary layer. Int. J. Numer. Methods Fluids 69(7), 1219-1236 (2012)

57. Jha, S.K.: Effect of Spatial Variability of Soil Properties on Slope Reliability Using Random Finite Element and First Order Second Moment Methods. Indian Geotech. J. 45(2), 145-155 (2015)

58. Jiang, S.H., Li, D.Q., Cao, Z.J., Zhou, C.B., Phoon, K.K.: Efficient System Reliability Analysis of Slope Stability in Spatially Variable Soils Using Monte Carlo Simulation. J. Geotech. Geoenviron. Eng. 141(2), 1-13 (2014)

59. Johari, A., Rezvani Pour, J., Javadi, A.: Reliability analysis of static liquefaction of loose sand using the random finite element method. Eng. Comput. 32(7), 2100-2119 (2015)

60. Johnson, M.E.: Multivariate statistical simulation: A guide to selecting and generating continuous multivariate distributions. John Wiley \& Sons, New York (2013)

61. Journel, A.G.: Geostatistics for conditional simulation of ore bodies. Economic Geology 69(5), 673-687 (1974)

62. Klammler, H., Hatfield, K., McVay, M., da Luz, J.A.G.: Approximate up-scaling of geospatial variables applied to deep foundation design. Georisk 5(3-4), 163-172 (2011)

63. Kozintsev, B.: Computations with gaussian random fields. Ph.D. thesis, University of Maryland, College Park (1999)

64. Kraichnan, R.H.: Diffusion by a random velocity field. Phys. Fluids 13(1), 22-31 (1970)

65. Lang, A., Potthoff, J.: Fast simulation of Gaussian random fields. Monte Carlo Methods Appl. 17(3), 1-15 (2011)

66. Lavorato, D., Vanzi, I., Nuti, C., Monti, G.: Generation of non-synchronous earthquake signals. In: Risk and Reliability Analysis: Theory and Applications, pp. 169-198. Springer (2017)

67. Le Goc, R., Bouzeran, L., Darcel, C., Ivars, D.M., et al.: Using correlated random fields for modeling the spatial heterogeneity of rock. In: ISRM Regional SymposiumEUROCK 2015. International Society for Rock Mechanics and Rock Engineering (2015)

68. Le Maître, O., Knio, O.M.: Spectral methods for uncertainty quantification: with applications to computational fluid dynamics. Springer Science \& Business Media (2010)

69. Le Ravalec, M., Noetinger, B., Hu, L.Y.: The fft moving average (fft-ma) generator: An efficient numerical method for generating and conditioning gaussian simulations. Math. Geol. 32(6), 701-723 (2000) 
70. Li, D.Q., Xiao, T., Cao, Z.J., Zhou, C.B., Zhang, L.M.: Enhancement of random finite element method in reliability analysis and risk assessment of soil slopes using Subset Simulation. Landslides 13(2), 293-303 (2016)

71. Li, H., Zhang, D.: Probabilistic collocation method for flow in porous media: Comparisons with other stochastic methods. Water Resour. Res. 43(9), 1-13 (2007)

72. Li, L., Chu, X.: Effect of 2-D Random Field Discretization on Failure Probability and Failure Mechanism in Probabilistic Slope Stability. Geotech. Geol. Eng. 34(2), 437-447 (2016)

73. Li, S.G., Liu, Q.: Interactive ground water (igw). Environ. Model. Softw. 21(3), 417$418(2006)$

74. Li, Y.J., Hicks, M.A., Vardon, P.J.: Uncertainty reduction and sampling efficiency in slope designs using 3D conditional random fields. Comput. Geotech. 79, 159-172 (2016)

75. Litvinova, E., Dolya, G.: Modeling of fluctuations of laser radiation scattered on the reflector array in a turbulent atmosphere. In: 2011 11th International Conference on Laser and Fiber-Optical Networks Modeling (LFNM), pp. 1-3. IEEE (2011)

76. Liu, K., Hicks, M.A., Vardon, P.J., Jommi, C.: Probabilistic analysis of velocity distribution under earth embankments for piping investigation. Geotechnical Safety and Risk V, edited by T. Schweckendiek, AF van Tol, D. Pereboom, M. van Staveren, and PMCBM Cools pp. 683-688 (2015)

77. Liu, Y., Hu, J., Li, Y.P., Li, L.H.: Statistical evaluation of the overall strength of a soil-cement column under axial compression. Constr. Build. Mater. 132, 51-60 (2017)

78. Liu, Y., Zhang, W., Zhang, L., Zhu, Z., Hu, J., Wei, H.: Probabilistic stability analyses of undrained slopes by $3 \mathrm{~d}$ random fields and finite element methods. Geosci. Front. 9(6), 1657-1664 (2018)

79. Loeve, M.: Probability theory. ii, volume 46 of. Graduate Texts in Mathematics (1978)

80. Mai, P.M., Beroza, G.C.: A spatial random field model to characterize complexity in earthquake slip. J. Geophys. Res. Solid Earth 107(B11), ESE 10-1-ESE 10-21 (2002)

81. Mantoglou, A.: Digital simulation of multivariate two- and three-dimensional stochastic processes with a spectral turning bands method. Math. Geol. 19(2), 129-149 (1987)

82. Mantoglou, A., Gelhar, L.W.: Stochastic modeling of large-scale transient unsaturated flow systems. Water Resour. Res. 23(1), 37-46 (1987)

83. Mantoglou, A., Wilson, J.L.: The Turning Bands Method for simulation of random fields using line generation by a spectral method. Water Resour. Res. 18(5), 1379$1394(1982)$

84. Massari, C., Yeh, T.C.J., Ferrante, M., Brunone, B., Meniconi, S.: Detection and sizing of extended partial blockages in pipelines by means of a stochastic successive linear estimator. J. Hydroinform. 16(2), 248 (2014)

85. Matheron, G.: The intrinsic random functions and their applications. Adv. Appl. Probab. 5(03), 439-468 (1973)

86. Mejía, J.M., Rodríguez-Iturbe, I.: On the synthesis of random field sampling from the spectrum: An application to the generation of hydrologic spatial processes. Water Resour. Res. 10(4), 705-711 (1974)

87. Miguel, L.F.F., Riera, J.D., Iturrioz, I.: Influence of size on the constitutive equations of concrete or rock dowels. Int. J. Numer. Anal. Methods Geomech. 32(15), 1857-1881 (2008)

88. Müller, W.G.: Collecting spatial data: optimum design of experiments for random fields. Springer Science \& Business Media (2007)

89. Noetinger, B., Hume, L., Chatelin, R., Poncet, P.: Effective viscosity of a random mixture of fluids. Phys. Rev. Fluids 3(1), 014,103 (2018)

90. Noh, H.C.: Plate response variability due to triple random parameters. KSCE J. Civ. Eng. 15(3), 517-526 (2011)

91. Noh, H.C., Park, T.: Response variability of laminate composite plates due to spatially random material parameter. Comput. Methods Appl. Mech. Eng. 200(29-32), 2397$2406(2011)$

92. Nuttall, J.D.: Parallel implementation and application of the random finite element method. Ph.D. thesis, The University of Manchester (United Kingdom) (2011)

93. Oliver, D.S.: Moving averages for Gaussian simulation in two and three dimensions. Math. Geol. 27(8), 939-960 (1995) 
94. Paiboon, J., Griffiths, D.V., Huang, J., Fenton, G.A.: Numerical analysis of effective elastic properties of geomaterials containing voids using $3 \mathrm{D}$ random fields and finite elements. Int. J. Solids Struct. 50(20-21), 3233-3241 (2013)

95. Pan, Q., Dias, D.: Probabilistic evaluation of tunnel face stability in spatially random soils using sparse polynomial chaos expansion with global sensitivity analysis. Acta Geotech. 12(6), 1415-1429 (2017)

96. Pardo-Iguzquiza, E.: The Fourier integral method: an efficient spectral method for simulation of random fields. Math. Geol. 25(2), 177 (1993)

97. Park, M.H., Tretyakov, M.: A block circulant embedding method for simulation of stationary gaussian random fields on block-regular grids. Int. J. Uncertain. Quantif. $\mathbf{5}(6)(2015)$

98. Pebesma, E., Graeler, B., Pebesma, M.E.: Package gstat (2018)

99. Pebesma, E.J.: Multivariable geostatistics in S: The gstat package. Comput. Geosci. 30(7), 683-691 (2004)

100. Podroužek, J., Vorel, J., Wan-Wendner, R.: Random and gradient based fields in discrete particle models of heterogeneous materials (2017)

101. Raabe, N., Thieler, A.M., Weihs, C., Fried, R., Rautert, C., Biermann, D.: Modeling Material Heterogeneity by Gaussian Random Fields for the Simulation of Inhomogeneous Mineral Subsoil Machining. SIMUL (c), 97-102 (2012)

102. Rahman, M.M., Nguyen, H.B.K.: Applications of Random Finite Element Method in Bearing Capacity Problems. ADVCOMP 2012 : The Sixth International Conference on Advanced Engineering Computing and Applications in Sciences Applications (c), $53-58(2012)$

103. Riahi, A., Hazzard, J., Lorig, L.: Heterogeneous Distribution of the Coefficient of Permeability and an Equivalent Homogeneous Approach. Proceedings 46th US Rock Mechanics held in Chicago, Illinois (360), 1-8 (2012)

104. Robin, M.J.L., Gutjahr, A.L., Sudicky, E.A., Wilson, J.L.: Cross-correlated random field generation with the direct Fourier Transform Method. Water Resour. Res. 29(7), 2385-2397 (1993)

105. Ruan, F., McLaughlin, D.: An efficient multivariate random field generator using the fast Fourier transform. Adv. Water Resour. 21(5), 385-399 (1998)

106. Rungbanaphan, P., Honjo, Y., Yoshida, I.: Settlement prediction by spatial-temporal random process using Asaoka's method. Georisk 4(4), 174-185 (2010)

107. Rungbanaphan, P., Honjo, Y., Yoshida, I.: Spatial-temporal prediction of secondary compression using random field theory. Soils Found. 52(1), 99-113 (2012)

108. Schlather, M., Malinowski, A., Oesting, M., Boecker, D., Strokorb, K., Engelke, S., Pfaff, B.: R core team (2017). randomfields: Simulation and analysis of random fields. r package version 3.1. 50 (2017)

109. Schlüter, S., Vogel, H.J.: On the reconstruction of structural and functional properties in random heterogeneous media. Adv. Water Resour. 34(2), 314-325 (2011)

110. Schlüter, S., Weller, U., Vogel, H.J.: Segmentation of X-ray microtomography images of soil using gradient masks. Comput. Geosci. 36(10), 1246-1251 (2010)

111. Schwab, C., Todor, R.A.: KarhunenLoève approximation of random fields by generalized fast multipole methods. J. Comput. Phys. 217(1), 100-122 (2006)

112. Shafei, B., Alipour, A.: Application of large-scale non-Gaussian stochastic fields for the study of corrosion-induced structural deterioration. Eng. Struct. 88, 262-276 (2015)

113. Shen, P., Zhang, L., Zhu, H.: Rainfall infiltration in a landslide soil deposit: Importance of inverse particle segregation. Eng. Geol. 205, 116-132 (2016)

114. Shinozuka, M.: Simulation of Multivariate and Multidimensional Random Processes. J. Acoust. Soc. Am. 49(1B), 357-368 (1971)

115. Shinozuka, M., Deodatis, G.: Simulation of Multi-Dimensional Gaussian Stochastic Fields by Spectral Representation. Appl. Mech. Rev. 49(1), 29 (1996)

116. Shinozuka, M., Jan, C.M.: Digital simulation of random processes and its applications. J. Sound Vib. 25(1), 111-128 (1972)

117. Smirnov, A., Shi, S., Celik, I.: Random Flow Generation Technique for Large Eddy Simulations and Particle-Dynamics Modeling. J. Fluids Eng. 123(2), 359 (2001)

118. Smith, I.M., Griffiths, D.V., Margetts, L.: Programming the finite element method. John Wiley \& Sons, New York (2013) 
119. Song, K.I., Cho, G.C., Lee, S.W.: Effects of spatially variable weathered rock properties on tunnel behavior. Probabilist. Eng. Mech. 26(3), 413-426 (2011)

120. Spencer, W.A.: Parallel stochastic and finite element modelling of clay slope stability in 3D. The University of Manchester (United Kingdom) (2007)

121. Srivastava, M.: Nicolas remy, alexandre boucher and jianbing wu: Applied geostatistics with sgems: A users guide (2009)

122. Stefanos, D.: Constitutive relations of stress and strain in stochastic finite element method. Amer. J. Math. 5(6), 164-173 (2015)

123. Stefanou, G.: The stochastic finite element method: past, present and future. Comput. Methods Appl. Mech. Eng. 198(9-12), 1031-1051 (2009)

124. Tang, X.S., Li, D.Q., Zhou, C.B., Phoon, K.K.: Copula-based approaches for evaluating slope reliability under incomplete probability information. Struct. Saf. 52, 90-99 (2015)

125. Teatini, P., Ferronato, M., Gambolati, G., Baú, D., Putti, M.: Anthropogenic Venice uplift by seawater pumping into a heterogeneous aquifer system. Water Resour. Res. 46(11), 1-16 (2010)

126. Tian, H., Xu, T., Li, Y., Yang, Z., Wang, F.: Evolution of sealing efficiency for $\mathrm{CO} 2$ geological storage due to mineral alteration within a hydrogeologically heterogeneous caprock. Appl. Geochem. 63, 380-397 (2015)

127. Tompson, A.F.B., Ababou, R., Gelhar, L.W.: Implementation of the three-dimensional turning bands random field generator. Water Resour. Res. 25(10), 2227-2243 (1989)

128. Vanmarcke, E.: Random fields: analysis and synthesis. World Scientific (2010)

129. Varkey, D., Hicks, M., Vardon, P.: Influence of Spatial Variability of Shear Strength Parameters on 3D Slope Reliability and Comparison of Analysis Methods. Georisk (GSP 284), 400-409 (2017)

130. Veganzones, M.A., Hernández, C.: On the use of a hybrid approach to contrast endmember induction algorithms. Lecture Notes in Computer Science (including subseries Lecture Notes in Artificial Intelligence and Lecture Notes in Bioinformatics) 6077 LNAI(PART 2), 69-76 (2010)

131. Vogel, H.J., Weller, U., Ippisch, O.: Non-equilibrium in soil hydraulic modelling. J. Hydrol. 393(1-2), 20-28 (2010)

132. Xenaki, A., Gerstoft, P., Mosegaard, K.: Modeling and detection of oil in sea water. J. Acoust. Soc. Am. 134(4), 2790-2798 (2013)

133. Xue, L., Dai, C., Wang, L.: Development of a general package for resolution of uncertainty-related issues in reservoir engineering. Energies 10(2), 197 (2017)

134. Zeng, X., Wang, D., Wu, J., Chen, X.: Reliability Analysis of the Groundwater Conceptual Model. Hum. Ecol. Risk Assess. 19(2), 515-525 (2013)

135. Zhang, D.: Stochastic methods for flow in porous media: coping with uncertainties. Elsevier (2001)

136. Zhang, D., Lu, Z.: An efficient, high-order perturbation approach for flow in random porous media via Karhunen-Loève and polynomial expansions. J. Comput. Phys. 194(2), 773-794 (2004)

137. Zhang, L.L., Cheng, Y., Li, J.H., Zhou, X.L., Jeng, D.S., Peng, X.Y.: Wave-induced oscillatory response in a randomly heterogeneous porous seabed. Ocean Eng. 111, 116-127 (2016)

138. Zhang, W., Goh, A.T.: Reliability assessment on ultimate and serviceability limit states and determination of critical factor of safety for underground rock caverns. Tunn. Undergr. Sp. Tech. 32, 221-230 (2012)

139. Zheng, Z., Dai, H.: Simulation of multi-dimensional random fields by KarhunenLoève expansion. Comput. Methods. Appl. Mech. Eng. 324, 221-247 (2017)

140. Zhu, D., Griffiths, D.V., Huang, J., Fenton, G.A.: Probabilistic stability analyses of undrained slopes with linearly increasing mean strength. Géotechnique 67(8), 733-746 (2017)

141. Zhu, H., Griffiths, D.V., Fenton, G.A., Zhang, L.: Undrained failure mechanisms of slopes in random soil. Eng. Geol. 191, 31-35 (2015) 
A Matlab code corresponding to Section 2

Here we provide the MATLAB code for four random field generation methods presented in Section 2. Interested readers may refer to the link https://github.com/YLiu-5/Random। textunderscoreField \textunderscoreGeneration. 